\title{
INTERPOLATING AND SAMPLING SEQUENCES FOR ENTIRE FUNCTIONS
}

\author{
NICOLAS MARCO, XAVIER MASSANEDA, AND JOAQUIM ORTEGA-CERDÀ
}

\begin{abstract}
We characterise interpolating and sampling sequences for the spaces of entire functions $f$ such that $f e^{-\phi} \in L^{p}(\mathbb{C}), p \geq 1$ where $\phi$ is a subharmonic weight whose Laplacian is a doubling measure. The results are expressed in terms of some densities adapted to the metric induced by $\Delta \phi$. They generalise previous results by Seip for the case $\phi(z)=|z|^{2}$, Berndtsson $\&$ Ortega-Cerdà and Ortega-Cerdà \& Seip for the case when $\Delta \phi$ is bounded above and below, and Lyubarskii \& Seip for 1-homogeneous weights of the form $\phi(z)=|z| h(\arg z)$, where $h$ is a trigonometrically strictly convex function.
\end{abstract}

\section{CONTENTS}

1. Introduction 2

\begin{tabular}{lll}
\hline 2. & Subharmonic functions with doubling Laplacian & 7
\end{tabular}

\begin{tabular}{lll}
\hline 2.1. & Doubling measures & 7
\end{tabular}

2.2. Local behaviour and regularisation of $\phi \quad 14$

\begin{tabular}{llr}
\hline 2.3. & Generalized weighted spaces & 18
\end{tabular}

\begin{tabular}{ll}
2.4. & The multiplier \\
\hline
\end{tabular}

\begin{tabular}{lll}
\hline 3. & Basic properties of functions in $\mathcal{F}_{\phi}^{p}$ & 21
\end{tabular}

3.1. Pointwise estimates 22

3.2. Hörmander type estimates 23

$\begin{array}{lll}3.3 . & \text { Bergman kernel estimates } & 24\end{array}$

$\begin{array}{lll}3.4 . & \text { Scaled translations and invariance } & 25\end{array}$

3.5. Weak limits. 27

4. Preliminary properties of sampling and interpolating sequences 29

4.1. Weak limits and interpolating and sampling sequences 30

Date: May 22, 2002.

1991 Mathematics Subject Classification. 30E05,46E20.

Key words and phrases. Interpolating sequences, Sampling sequences, Entire functions.

The authors are supported by the Research Training Network "Analysis and Operators", with contract number HPRN-CT-2000-00116. The last two authors are supported by the DGICYT grant BFM2002-04072-C02-01 and by the CIRIT grant 2001SGR 00172. 
4.2. Non-existence of simultaneously sampling and interpolating sequences 32

4.3. Inclusions between various spaces of interpolating sequences 35

4.4. Inclusions between various spaces of sampling sequences 36

$\begin{array}{lll}4.5 . & \text { Nets } & 38\end{array}$

$\begin{array}{lll}\text { 5. } & \text { Sufficient conditions for sampling } & 40\end{array}$

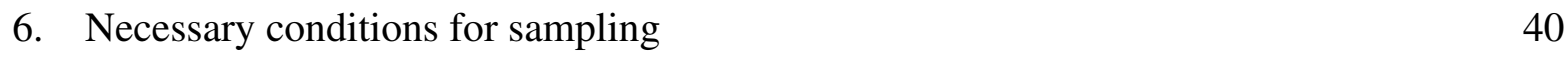

\begin{tabular}{lll}
\hline 7. & Sufficient conditions for interpolation & 42
\end{tabular}

\begin{tabular}{lll}
\hline 8. Necessary conditions for interpolation & 45
\end{tabular}

\begin{tabular}{|ll|l|}
\hline Appendix. Alternative construction of peak functions. (Theorem & 180 & 47
\end{tabular}

$\begin{array}{ll}\text { References } & 50\end{array}$

\section{INTRODUCTION}

In this paper we provide Beurling-type density conditions for sampling and interpolation in certain generalised Fock spaces. We consider a rather general situation, with only mild regularity conditions on the possible growth. Let $\phi$ be a (nonharmonic) subharmonic function whose Laplacian $\Delta \phi$ is a doubling measure (see definition and properties in Section 2.1). The spaces we deal with are as follows:

$$
\begin{aligned}
\mathcal{F}_{\phi}^{p} & =\left\{f \in H(\mathbb{C}):\|f\|_{\mathcal{F}_{\phi}^{p}}^{p}=\int_{\mathbb{C}}|f|^{p} e^{-p \phi} \rho^{-2}<\infty\right\} \quad 1 \leq p<\infty, \\
\mathcal{F}_{\phi}^{\infty} & =\left\{f \in H(\mathbb{C}):\|f\|_{\mathcal{F}_{\phi}^{\infty}}=\sup _{z \in \mathbb{C}}|f(z)| e^{-\phi(z)}<\infty\right\} .
\end{aligned}
$$

The function $\rho^{-2}$ is a regularised version of $\Delta \phi$, as described in [Chr91]. More precisely, if $\mu=\Delta \phi$ and $z \in \mathbb{C}$, then $\rho_{\phi}(z)$ (or simply $\rho(z)$ if no confusion can arise) denotes the positive radius such that $\mu(D(z, \rho(z))=1$. Such a radius exists because doubling measures have no mass on circles.

Canonical examples of the weights considered are $\phi(z)=|z|^{\beta}$, with $\beta>0$.

There are other spaces of functions that although at first sight do not seem to be covered by our results, can be conveniently adjusted so that the theorems apply. This the case of the weighted spaces of holomorphic functions

$$
\left\{f \in H(\mathbb{C}): \int_{\mathbb{C}}|f|^{p} e^{-p \phi} \rho^{\alpha}<\infty\right\},
$$

with $\phi$ a subharmonic function with doubling Laplacian and $\alpha \in \mathbb{R}$. For any such space there exists a subharmonic function $\psi$ with doubling Laplacian such that

$$
C^{-1} e^{-p \phi} \rho^{\alpha} \leq e^{-p \psi} \leq C e^{-p \phi} \rho^{\alpha}, \quad \text { and } \quad C^{-1} \rho_{\psi} \leq \rho_{\phi} \leq C \rho_{\psi}
$$


for some $C>1$, see subsection 2.3. Therefore the weighted space coincides with $\mathcal{F}_{\psi}^{p}$.

Among these two particular families of spaces seem of special interest. The first one are the usual weighted $L^{p}$-spaces of entire functions, obtained with $\alpha=0$. The second case arises when $\alpha=-2 ;$ the standard $\mathcal{F}_{\phi}^{p}$ spaces, they coincide with

$$
\left\{f \in H(\mathbb{C}): \int_{\mathbb{C}}|f|^{p} e^{-p \phi} \Delta \phi<\infty\right\} .
$$

Another source of examples comes from the problem studied in [LS94]. Given a bounded smooth convex set $G$ in $\mathbb{C}$, consider the Smirnov (Hardy) space $E^{2}(G)$, the closure of the analytic polynomials with the topology induced by the norm

$$
\|f\|_{E^{2}(G)}=\int_{\partial G}|f(z)|^{2}|d z| .
$$

When the curvature of $\partial G$ is bounded from above and below, there exists a Paley-Wiener type theorem, due to Lyubarskiı [Lyu88], that gives an isomorphism between $E^{2}(G)$ and the space $\mathcal{F}_{G}$ of entire functions $f$ such that

$$
\|f\|^{2}=\int_{\mathbb{C}}|f(z)|^{2} e^{-2 k(\arg (z))|z|}|z|^{-1 / 2} d m(z)<\infty .
$$

Here $k$ is the so-called support function of $G$. By the isometry, the description of frames or Riesz families of exponentials in the Smirnov space is equivalent to the description of sampling or interpolating sequences in $\mathcal{F}_{G}$, which was was achieved in [LS94]. The spaces $\mathcal{F}_{G}$ are of the sort that we are considering, since $\phi(z)=|z| k(\arg (z))$ has doubling Laplacian and $\rho(z) \simeq \sqrt{|z|}$. It seems plausible that our spaces also cover some cases where the curvature vanishes polinomially at some point, via the general Paley-Wiener theorem due to Lutsenko and Yulmukhametov (see [LY91]). In this theorem there is a description of the Fourier Laplace transform of the Smirnov space. The space they describe is not directly one of our $\mathcal{F}_{\phi}^{2}$, but it seems likely that it can be norm-equivalent to one of them (similiarly to subsection 2.3), at least in the case where the curvature vanishes polinomially at some point.

Since functions $f$ in the spaces $\mathcal{F}_{\phi}^{p}$ are determined by the growth of $|f|$, their restriction to a sequence should be described as well in terms of growth.

Let $\Lambda \subset \mathbb{C}$ be a sequence and let $v=\left\{v_{\lambda}\right\}_{\lambda \in \Lambda}$ be an associated sequence of values.

Definition 1. A sequence $\Lambda$ is an interpolating sequence for $\mathcal{F}_{\phi}^{p}, 1 \leq p<\infty$ (denoted $\Lambda \in$ Int $\mathcal{F}_{\phi}^{p}$ ), if for every sequence of values $v$ such that

$$
\|v\|_{\ell_{\phi}^{p}(\Lambda)}^{p}=\sum_{\lambda \in \Lambda}\left|v_{\lambda}\right|^{p} e^{-p \phi(\lambda)}<\infty
$$

there exists $f \in \mathcal{F}_{\phi}^{p}$ such that $f \mid \Lambda=v$.

Also, $\Lambda \in \operatorname{Int} \mathcal{F}_{\phi}^{\infty}$ if for every sequence of values $v$ such that

$$
\|v\|_{\ell_{\phi}^{\infty}(\Lambda)}=\sup _{\lambda \in \Lambda}\left|v_{\lambda}\right| e^{-\phi(\lambda)}<\infty
$$


there exists $f \in \mathcal{F}_{\phi}^{\infty}$ such that $f \mid \Lambda=v$.

An application of the closed graph theorem shows that when $\Lambda \in \operatorname{Int} \mathcal{F}_{\phi}^{p}$ there is $M>0$ such that for any $v \in \ell_{\phi}^{p}(\Lambda)$, there exists $f \in \mathcal{F}_{\phi}^{p}$ with $f \mid \Lambda=v$ and

$$
\|f\|_{\mathcal{F}_{\phi}^{p}} \leq M\|v\|_{\ell_{\phi}^{p}(\Lambda)} .
$$

The least possible $M$ in (1) is called the interpolating constant of $\Lambda$ and is denoted by $M_{\phi}^{p}(\Lambda)$, or $M(\Lambda)$ if no confusion is possible.

Definition 2. A sequence $\Lambda$ is a sampling sequence for $\mathcal{F}_{\phi}^{p}, 1 \leq p<\infty\left(\right.$ denoted $\Lambda \in \operatorname{Samp} \mathcal{F}_{\phi}^{p}$ ), if there exists $C>0$ such that for every $f \in \mathcal{F}_{\phi}^{p}$

$$
C^{-1}\left\|f\left|\Lambda\left\|_{\ell_{\phi}^{p}(\Lambda)} \leq\right\| f\left\|_{\mathcal{F}_{\phi}^{p}} \leq C\right\| f\right| \Lambda\right\|_{\ell_{\phi}^{p}(\Lambda)} .
$$

Also, $\Lambda \in \operatorname{Samp} \mathcal{F}_{\phi}^{\infty}$ if there exists $C>0$ such that for every $f \in \mathcal{F}_{\phi}^{\infty}$

$$
\|f\|_{\mathcal{F}_{\phi}^{\infty}} \leq C\|f \mid \Lambda\|_{\ell_{\phi}^{\infty}(\Lambda)} .
$$

The least constant $C$ verifying these inequalities is called the sampling constant of $\Lambda$ and is denoted $L_{\phi}^{p}(\Lambda)$, or simply $L(\Lambda)$.

The definitions of interpolating and sampling sequences in the spaces defined by $L^{\infty}$ norms reflect the maximal growth for functions in the space, and are natural. The definition for $p<\infty$ can be motivated in the following way. Consider for instance the case $p=2$. The estimates of the normalised Bergman kernel $k_{\phi}(\lambda, z)$ in $\mathcal{F}_{\phi}^{2}$ (see Lemma 21) show that $\left\langle k_{\phi}(\lambda, \cdot), f\right\rangle \simeq f(\lambda) e^{-\phi(\lambda)}$ for all $f \in \mathcal{F}_{\phi}^{2}$. Thus $\Lambda \in \operatorname{Samp} \mathcal{F}_{\phi}^{2}$ if and only if

$$
\|f\|_{\mathcal{F}_{\phi}^{2}} \simeq \sum_{\lambda \in \Lambda}\left|\left\langle k_{\phi}(\lambda, \cdot), f\right\rangle\right|^{2} \quad \text { for all } f \in \mathcal{F}_{\phi}^{2},
$$

that is, if and only if $\left\{k_{\phi}(\lambda, \cdot)\right\}_{\lambda \in \Lambda}$ is a frame in $\mathcal{F}_{\phi}^{2}$. Similarly, $\Lambda \in \operatorname{Int} \mathcal{F}_{\phi}^{2}$ if and only if $\left\{k_{\phi}(\lambda, \cdot)\right\}_{\lambda \in \Lambda}$ is a Riesz basis in its closed linear span in $\mathcal{F}_{\phi}^{2}$. These are the standard problems of interpolation and sampling in Hilbert spaces of functions with reproducing kernels [SS61]. For $p \neq 2$ the previous definitions give the appropriate notions of interpolation and sampling as well, in view of the pointwise growth of functions in the spaces (see Lemma 19 and Remark 6).

Our description of interpolating and sampling sequences is expressed in terms of certain Beurling-type densities adapted to the metric induced by $\Delta \phi$, or more precisely, by its regularisation $\rho^{-2}(z) d z \otimes d \bar{z}$. Before introducing the densities we need the notion of $\rho$-separation.

Definition 3. A sequence $\Lambda$ is $\rho$-separated if there exists $\delta>0$ such that

$$
\left|\lambda-\lambda^{\prime}\right| \geq \delta \max \left(\rho(\lambda), \rho\left(\lambda^{\prime}\right)\right) \quad \lambda \neq \lambda^{\prime} .
$$

This is equivalent to saying that the points in $\Lambda$ are separated by a fixed distance in the metric above (Lemma 4). 
Definition 4. Assume that $\Lambda$ is a $\rho$-separated sequence and denote $\mu=\Delta \phi$.

The upper uniform density of $\Lambda$ with respect to $\Delta \phi$ is

$$
\mathcal{D}_{\Delta \phi}^{+}(\Lambda)=\limsup _{r \rightarrow \infty} \sup _{z \in \mathbb{C}} \frac{\#(\Lambda \cap \overline{D(z, r \rho(z))})}{\mu(D(z, r \rho(z)))} .
$$

The lower uniform density of $\Lambda$ with respect to $\Delta \phi$ is

$$
\mathcal{D}_{\Delta \phi}^{-}(\Lambda)=\liminf _{r \rightarrow \infty} \inf _{z \in \mathbb{C}} \frac{\#(\Lambda \cap \overline{D(z, r \rho(z))})}{\mu(D(z, r \rho(z)))} .
$$

The main theorems are the following.

Theorem A. A sequence $\Lambda$ is sampling for $\mathcal{F}_{\phi}^{p}, p \in[1, \infty)$, if and only if $\Lambda$ is a finite union of $\rho$-separated sequences containing a $\rho$-separated subsequence $\Lambda^{\prime}$ such that $\mathcal{D}_{\Delta \phi}^{-}\left(\Lambda^{\prime}\right)>1 / 2 \pi$. A sequence $\Lambda$ is sampling for $\mathcal{F}_{\phi}^{\infty}$ if and only if $\Lambda$ contains a $\rho$-separated subsequence $\Lambda^{\prime}$ such that $\mathcal{D}_{\Delta \phi}^{-}\left(\Lambda^{\prime}\right)>1 / 2 \pi$.

Theorem B. A sequence $\Lambda$ is interpolating for $\mathcal{F}_{\phi}^{p}, p \in[1, \infty]$, if and only if $\Lambda$ is $\rho$-separated and $\mathcal{D}_{\Delta \phi}^{+}(\Lambda)<1 / 2 \pi$.

In particular, there are no sequences which are simultaneously sampling and interpolating (it should be mentioned that this is not obtained as a corollary of the theorems; it is actually an important ingredient of the proofs).

These results generalise previous work, beginning with the papers by Seip and Seip-Wallstén [Sei92], [SW92]. They described the interpolating and sampling sequences for the classical Fock space in terms of the so-called Nyquist densities. In the notation above this corresponds to $\phi(z)=|z|^{2}$. This was extended in [BOC95] and [OCS98] to the case of entire functions $f$ such that $f e^{-\phi} \in L^{p}(\mathbb{C})$, where $\phi$ is subharmonic with bounded Laplacian $\varepsilon<\Delta \phi<M$. The description was given again in terms of some Nyquist type densities. In these cases the function $\rho$ is bounded above and below, hence the metric $\rho^{-2}(z) d z \otimes d \bar{z}$ is equivalent to the Euclidean metric. In particular, $\rho(z)$ can be replaced by the constant 1 in the definition of the uniform densities. In [LS94] the authors provided a description, in terms of directional densities, of sampling and interpolating sequences for the spaces with 1-homogeneous weights of the form $\phi(z)=|z| h(\arg z)$, where $h$ is a trigonometrically strictly convex function.

There are also some partial results in several complex variables. The classical Fock space has been studied in [MT00] and the weighted scenario in [Lin01]. In this context there exist necessary or sufficient density conditions, which do not completely characterise the sampling or interpolating sequences.

Interpolation problems for other spaces of functions related to these weights have been considered by Squires and Berenstein and Li (see for instance [Squ83], [BL95] and the references therein). 
The results mentioned above relied on the remarkable work by Beurling [Beu89] and on Hörmander's weighted $L^{2}$-estimates for the $\bar{\partial}$ equation [Hör94]. In our proofs we first extend Beurling's tools to the context of certain spaces which are non-invariant under translations. This is in the spirit of [LS94] where they already considered some limiting spaces of the original space deformed by translations. We need as well a Hörmander type theorem giving precise estimates for the $\bar{\partial}$ equation in Banach norms other than $L^{2}$.

The plan of the paper is the following: In Section 2 we study the properties of doubling measures. Recall that the only assumption on our subharmonic weight $\phi$ is that the measure $\Delta \phi$ is doubling. We will need a regularisation of $\phi$ and the construction of a multiplier associated to $\phi$ (that is, an entire function $f$ such that $|f|$ approximates $e^{\phi}$ ), very much in the spirit of [LM01] and [OC99].

In Section 3 we state and prove some basic properties of functions in $\mathcal{F}_{\phi}^{p}$. The main result in this section is the following Hörmander type theorem.

Theorem C. Let $\phi$ be a subharmonic function such that $\Delta \phi$ is a doubling measure. There is a solution $u$ to the equation $\bar{\partial} u=f$ such that $\left\|u e^{-\phi}\right\|_{L^{p}(\mathbb{C})} \lesssim\left\|f e^{-\phi} \rho\right\|_{L^{p}(\mathbb{C})}$ for any $1 \leq p \leq \infty$.

We also include the estimates of the Bergman kernel that justify the notion of interpolating and sampling sequences we have considered. Finally, we study the invariance of our spaces under some appropriate scaled translations. This leads to the notion of weak limit and the corresponding analysis analogous to Beurling's.

Section 4 is devoted to some preliminary (but important) properties of interpolating and sampling sequences, including their behaviour under weak limits. The main results in this section are some inclusion relations between various spaces of interpolating and sampling sequences, and the fact that there are no sequences which are simultaneously interpolating and sampling for the same space of functions $\mathcal{F}_{\phi}^{p}$.

In Section 5 we prove the sufficiency part of Theorem A. We use again an approach similar to that of Beurling.

Section 6 includes the proof of the necessity part of Theorem A. For this we need once more Beurling's analysis, plus the non-existence of sampling and interpolating sequences. We use some theorems that relate the densities of sampling and interpolating sequences, following the ideas by Ramanathan and Steger [RS95].

Section 8 is devoted to the proof of the necessity part of Theorem B. We use Ramanathan and Steger's theorem plus an original argument that shows that the density inequality is actually strict.

Finally, in Section 7]we deal with the sufficiency part of Theorem B. In the course of the proof, whose main tool is the multiplier, we need to express the density in terms of rectangles instead of disks. The usual argument of Landau [Lan67] does not work, in view of the inhomogeneity of our measures. Theorem 43 takes care of this. 
A final word on notation: $C$ denotes a finite constant that may change in value from one occurrence to the next. The expression $f \lesssim g$ means that there is a constant $C$ independent of the relevant variables such that $f \leq C g$, and $f \simeq g$ means that $f \lesssim g$ and $g \lesssim f$.

Acknowledgement: We want to express our sincere gratitude to the referee for pointing out some improvements and simplifications that have enhanced the overall readability of the text.

\section{SUBHARMONIC FUNCTIONS WITH DOUBLING LAPLACIAN}

In this chapter we recap some results on doubling measures and subharmonic functions $\phi$ whose Laplacian $\Delta \phi$ is doubling. We start with regularity and integrability conditions on doubling measures. Next we show that $\phi$ can be regularised, in the sense that there exists $\psi$ subharmonic and regular for which the interpolation and sampling problems for $\mathcal{F}_{\phi}^{p}$ and $\mathcal{F}_{\psi, \alpha}^{p}$ are equivalent. The final part is dedicated to the construction of the multiplier. A useful application of this is the existence of holomorphic "peak functions" with controlled growth.

Definition 5. A nonnegative Borel measure $\mu$ in $\mathbb{C}$ is called doubling if there exists $C>0$ such that

$$
\mu(D(z, 2 r)) \leq C \mu(D(z, r))
$$

for all $z \in \mathbb{C}$ and $r>0$. We denote by $C_{\mu}$ the infimum of the constants $C$ for which the inequality holds.

Recall that when $\phi$ is subharmonic $\Delta \phi$ is a nonnegative Borel measure, finite on compact sets.

For convenience we write $D^{r}(z)=D(z, r \rho(z))$ and $D(z)=D^{1}(z)$. We will write $D_{\phi}^{r}(z)$ when we need to stress that the radius depends on $\phi$.

Henceforth $d m$ denotes the Lebesgue measure in $\mathbb{C}$. We also use the measure $d \sigma=d m / \rho^{2}$, which should be thought of as a doubling regularisation of $\Delta \phi$ (see Theorem 14).

2.1. Doubling measures. Throughout this section we assume that $\mu$ is a positive doubling measure non-identically zero. We begin with a result of Christ [Chr91, Lemma 2.1].

Lemma 1. Let $\mu$ be a doubling measure in $\mathbb{C}$. There exists $\gamma>0$ such that for any disks $D, D^{\prime}$ of respective radius $r(D)>r\left(D^{\prime}\right)$ with $D \cap D^{\prime} \neq \emptyset$ :

$$
\left(\frac{\mu(D)}{\mu\left(D^{\prime}\right)}\right)^{\gamma} \lesssim \frac{r(D)}{r\left(D^{\prime}\right)} \lesssim\left(\frac{\mu(D)}{\mu\left(D^{\prime}\right)}\right)^{1 / \gamma}
$$

In particular, the support of $\mu$ has positive Hausdorff dimension.

Remark 1. This implies that for all $z \in \mathbb{C}$ and $r>1$

$$
r^{\gamma} \lesssim \mu\left(D^{r}(z)\right) \lesssim r^{1 / \gamma}
$$

Also, applying Lemma 1 and (4) to $D(0,|z|)$ and $D(z)$ we have, for $\rho(z) \leq|z|$

$$
\frac{1}{|z|^{1 / \gamma^{2}}} \lesssim\left(\frac{1}{\mu(D(0,|z|))}\right)^{1 / \gamma} \lesssim \frac{\rho(z)}{|z|} \lesssim\left(\frac{1}{\mu(D(0,|z|))}\right)^{\gamma} \lesssim \frac{1}{|z|^{\gamma^{2}}}
$$


On the other hand, if $|z|<\rho(z)$, then $0 \in D(z)$. Thus Lemma 1 implies $\rho(z) \simeq \rho(0)$, hence $|z|<C$. Therefore, there exist $\eta, C_{0}>0$ and $\beta \in(0,1)$ such that

$$
C_{0}^{-1}|z|^{-\eta} \leq \rho(z) \leq C_{0}|z|^{\beta} \quad|z|>1
$$

Let us study in more detail the relationship between $\rho(z)$ and $\rho(\zeta)$ for various $z, \zeta \in \mathbb{C}$. A first observation is that $\rho(z)$ is a Lipschitz function. More precisely

$$
|\rho(z)-\rho(\zeta)| \leq|z-\zeta| \quad z, \zeta \in \mathbb{C} .
$$

To see this there is no loss of generality in assuming that $z, \zeta \in \mathbb{R}, \zeta<z$ and $\rho(\zeta)<\rho(z)$. Then $\zeta-\rho(\zeta)<z-\rho(z)$, since otherwise $D(\zeta) \subset D(z)$, contradicting the fact that $\mu(D(z))=$ $\mu(D(\zeta))=1$.

Lemma 2. [Chr91, p.205]. If $\zeta \notin D(z)$ then

$$
\frac{\rho(z)}{\rho(\zeta)} \lesssim\left(\frac{|z-\zeta|}{\rho(\zeta)}\right)^{1-\delta}
$$

for some $\delta \in(0,1)$ depending only on the doubling constant $C_{\mu}$.

As a consequence of Lemma 1 and (5) we have

Corollary 3. For every $r>1$ there exists $\gamma>0$ such that if $\zeta \in D^{r}(z)$ then

$$
\frac{1}{r^{\gamma}} \lesssim \frac{\rho(z)}{\rho(\zeta)} \lesssim r^{\gamma}
$$

It will be convenient to express some of the results in terms of the distance $d_{\phi}$ induced by the metric $\rho^{-2}(z) d z \otimes d \bar{z}$.

Lemma 4. There exists $\delta \in(0,1)$ such that for every $r>0$ there exists $C_{r}>0$ such that

(a) $C_{r}^{-1} \frac{|z-\zeta|}{\rho(z)} \leq d_{\phi}(z, \zeta) \leq C_{r} \frac{|z-\zeta|}{\rho(z)}$ if $|z-\zeta| \leq r \rho(z)$.

(b) $C_{r}^{-1}\left(\frac{|z-\zeta|}{\rho(z)}\right)^{\delta} \leq d_{\phi}(z, \zeta) \leq C_{r}\left(\frac{|z-\zeta|}{\rho(z)}\right)^{2-\delta}$ if $|z-\zeta|>r \rho(z)$.

This shows, in particular, that a sequence $\Lambda$ is $\rho$-separated if and only if there exists $\delta>0$ such that $\inf _{\lambda \neq \lambda^{\prime}} d_{\phi}\left(\lambda, \lambda^{\prime}\right)>\delta$.

Proof. By definition

$$
d_{\phi}(z, \zeta)=\inf \int_{0}^{1}\left|\gamma^{\prime}(t)\right| \rho^{-1}(\gamma(t)) d t
$$

where the infimum is taken over all piecewise $\mathcal{C}^{1}$ curves $\gamma:[0,1] \rightarrow \mathbb{C}$ with $\gamma(0)=z$ and $\gamma(1)=\zeta$.

The lower inequalities are contained in [Chr91, Lemma 3.1] and its proof. 
The upper estimate in case (a) is immediate from Corollary 3 . In case (b) take $\gamma(t)=z+$ $t(\zeta-z)$ and use Lemma 2 ; then

$$
d_{\phi}(z, \zeta) \leq|\zeta-z| \int_{0}^{1} \frac{d t}{\rho(\gamma(t))} \lesssim \int_{0}^{1} \frac{(t|\zeta-z|)^{1-\delta}}{(\rho(z))^{2-\delta}} d t \lesssim\left(\frac{|z-\zeta|}{\rho(z)}\right)^{2-\delta}
$$

From now on, given $z \in \mathbb{C}$ and $r>0$, we denote

$$
B(z, r)=\left\{\zeta \in \mathbb{C}: d_{\phi}(z, \zeta)<r\right\} .
$$

Doubling measures satisfy certain integrability conditions.

Lemma 5. Let $\mu$ be a doubling measure. There exist $C>0$ and $m \in \mathbb{N}$ depending on $C_{\mu}$ such that for any $r>0$

(a) $\int_{D(z, r)} \log \left(\frac{2 r}{|z-\zeta|}\right) d \mu(\zeta) \leq C \mu(D(z, r)) \quad z \in \mathbb{C}$.

(b) $\sup _{z \in \mathbb{C}} \int_{\mathbb{C}} \frac{d \mu(\zeta)}{1+d_{\phi}^{m}(z, \zeta)}<\infty$.

Proof. (a) is [Chr91, Lemma 2.3].

(b) According to Lemma 4 it is enough to consider the integral on $|z-\zeta| \geq r \rho(z)$. Applying Fubini's theorem we see that

$$
\begin{aligned}
& \int_{\zeta \notin D^{r}(z)}\left(\frac{\rho(z)}{|z-\zeta|}\right)^{m} d \mu(\zeta)=\int_{\zeta \notin D^{r}(z)} m \int_{0}^{\rho(z) /|z-\zeta|} t^{m-1} d t d \mu(\zeta) \\
&=m \int_{0}^{1 / r} t^{m-1} \int_{t<\rho(z) /|z-\zeta|<1 / r} d \mu(\zeta) d t \leq m \int_{0}^{1 / r} t^{m-1} \mu\left(D^{1 / t}(z)\right) d t .
\end{aligned}
$$

Then by (4) $\mu\left(D^{1 / t}(z)\right) \lesssim 1 / t^{k}$ for some $k>0$. Hence the integral is bounded if $m>k$.

This and Lemma 4(b) show that the result holds for $m$ big enough.

Remark 2. It is clear from the proof that

(b') $\lim _{r \rightarrow \infty} \sup _{z \in \mathbb{C}} \int_{\zeta \notin B(z, r)} \frac{d \mu(\zeta)}{d_{\phi}^{m}(z, \zeta)}=0$.

There is a discrete version of the previous Lemma.

Lemma 6. Let $\Lambda$ be a $\rho$-separated sequence. There exists $m \in \mathbb{N}$ such that

$$
\sup _{z \in \mathbb{C}} \sum_{\lambda \in \Lambda} \frac{1}{1+d_{\phi}^{m}(z, \lambda)}<\infty
$$


Proof. By the separation and Lemma 4, it is enough to see that for $m$ big enough

$$
\sup _{z \in \mathbb{C}} \sum_{\lambda \notin B(z, r)}\left(\frac{\rho(\lambda)}{|z-\lambda|}\right)^{m}<\infty
$$

Take $\delta>0$ such that the balls $\{B(\lambda, \delta)\}_{\lambda \in \Lambda}$ are pairwise disjoint. By Corollary 3

$$
\sum_{\lambda \notin B(z, r)}\left(\frac{\rho(\lambda)}{|z-\lambda|}\right)^{m} \lesssim \sum_{\lambda \notin B(z, r)} \int_{B(\lambda, \delta)}\left(\frac{\rho(\zeta)}{|z-\zeta|}\right)^{m} d \mu(\zeta) \lesssim \int_{\lambda \notin B(z, r)}\left(\frac{\rho(\zeta)}{|z-\zeta|}\right)^{m} d \mu(\zeta) .
$$

Lemma 5(b) implies that the integral is bounded.

For later use, we state a refinement that follows similarly from Remark 2 .

Corollary 7. Let $\Lambda$ be a $\rho$-separated sequence. There exists $m \in \mathbb{N}$ such that

$$
\lim _{r \rightarrow \infty} \sup _{z \in \mathbb{C}} \sum_{\lambda \notin B(z, r)} \frac{1}{d_{\phi}^{m}(z, \lambda)}=0 .
$$

We will need to partition the plane in rectangles of constant mass. We do that by adapting a general result of [Yul85] to our setting (see also [Dra01, Theorem 2.1]).

Theorem 8. Let $\mu$ be a positive doubling measure non-identically zero. There exists a "partition" of $\mathbb{C}$ in rectangles $R_{k}$ with sides parallel to the coordinate axis such that:

(a) $\mu=\sum_{k} \mu_{k}$, where $\mu_{k}:=\mu_{\mid R_{k}}$ satisfy $\mu_{k}(\mathbb{C})=1$.

(b) $R_{k}$ are quasi-squares: there exists a ratio e $>1$ depending only on $C_{\mu}$ such that the ratio of sides of each $R_{k}$ lies in the interval $[1 / e, e]$.

(c) There exists $C<0$ such that $C^{-1} \rho\left(a_{k}\right) \leq \operatorname{diam}\left(R_{k}\right) \leq C \rho\left(a_{k}\right)$, where $a_{k}$ denotes the centre of $R_{k}$.

(d) $\bigcup_{k} \overline{R_{k}}=\mathbb{C}$ and the interiors of $R_{k}$ are pairwise disjoint.

Remark 3. Dividing the original measure by $s \in \mathbb{R}^{+}$we obtain a partition of $\mathbb{C}$ into quasi-squares of mass $s$.

Proof. It is enough to partition the plane in quasi-squares of constant entire mass, because by an stopping-time argument of [OC99] these can then be split into quasi-squares of mass 1.

We construct our partition recursively. We start with a rectangle centred at 0 of entire mass, and with sidelengths $l \leq L$ so that $l \geq L / 2$ and $l^{1-\beta} \geq 12 \sqrt{2} C_{0}$, where $\beta$ and $C_{0}$ are given in (5) (rectangle $A B C D$ in the picture). Consider next a square $Q_{1}$ centred at 0 of sidelength $3 L$ ( $A_{1} B_{1} C_{1} D_{1}$ in the picture) and define $R$ as the quasi-square with vertices $A B B^{\prime} A^{\prime}$, where $A^{\prime}$ and $B^{\prime}$ are points on the same side of $Q_{1}$ taken so that $0 \notin R$. We want to make $R$ a little bigger, to make sure that its mass is entire, and we want to do that keeping control on the ratio of sides. Consider the rectangle $A B \tilde{B} \tilde{A}$, where $\tilde{A}, \tilde{B}$ are taken with $|A \tilde{A}|=|B \tilde{B}|=2\left|A A^{\prime}\right|$. Denote by $R^{\prime}$ the rectangle $A^{\prime} B^{\prime} \tilde{B} \tilde{A}$ added to $R$. For $\lambda \in R^{\prime}$,

$$
\frac{\rho(\lambda)}{l} \leq \frac{6 \sqrt{2} \rho(\lambda)}{|\lambda|} \leq \frac{6 \sqrt{2} C_{0}}{|\lambda|^{1-\beta}} \leq \frac{6 \sqrt{2} C_{0}}{l^{1-\beta}} \leq \frac{1}{2} .
$$


Since the sides of $R^{\prime}$ have length bigger or equal than $l$ we deduce that $R^{\prime}$ contains a disk of centre $\lambda$ and radius $\rho(\lambda)$, hence its mass is at least 1 . This shows that there exists a rectangle $R_{1}$ ( $A A^{\prime \prime} B^{\prime \prime} B$ in the picture) of entire mass between the original $R$ and the "doubled" $R$ '.

We finish the first step of the process by constructing the analogous quasi-square $R_{2}$ of entire mass at the opposite side of $R\left(C C^{\prime \prime} D^{\prime \prime} D\right.$ in the picture).

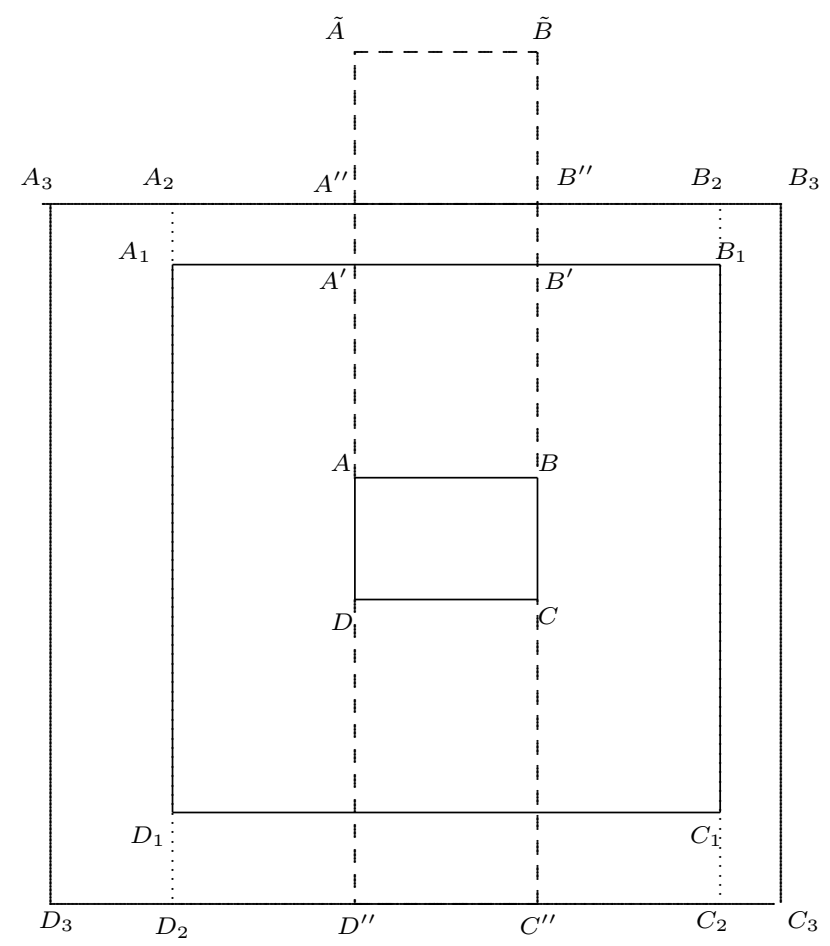

Consider next the rectangle $Q_{2}$ limited by the segments $\left(A^{\prime \prime} B^{\prime \prime}\right),\left(C^{\prime \prime} D^{\prime \prime}\right),\left(B_{1} C_{1}\right),\left(D_{1} A_{1}\right)$ (the rectangle $A_{2} B_{2} C_{2} D_{2}$ in the picture). We iterate the process above to each of the rectangles $B^{\prime \prime} B_{2} C_{2} C^{\prime \prime}$ and $D^{\prime \prime} D_{2} A_{2} A^{\prime \prime}$, thus obtaining two new quasi-squares $R_{3}=B^{\prime \prime} B_{3} C_{3} C^{\prime \prime}$ and $R_{4}=$ $D^{\prime \prime} D_{3} A_{3} A^{\prime \prime}$ of entire mass.

All in all, we obtain a new quasi-square $Q_{3}:=A_{3} B_{3} C_{3} D_{3}$ with ratio of sides lying in $[1 / 2,2]$ which is a disjoint union of 5 quasi-squares of entire mass. From here we repeat the process, taking $Q_{3}$ in place of the original $R$, and continue recursively to obtain the "partition" of $\mathbb{C}$. By construction we have (a), (b) and (d).

To prove (c) assume that $R$ is a quasi-square of mass 1 , centre $a$ and sidelengths $l, L$. Here $R \subset$ $D(a, L \sqrt{2})$, hence $\rho(a) \gtrsim L \gtrsim \operatorname{diam}(R)$. Also, $D(a, l) \subset R$ and $\operatorname{diam}(R) \lesssim l \lesssim \rho(a)$.

Lemmas 1 and 2 give control on how big a disc $D^{r}(\zeta)$ can be when $\zeta \in D^{s}(z)$. We will need another result along the same lines. 
Given a doubling measure $\mu$ and given $z \in \mathbb{C}$ and $0<r<s$, consider the associated regions

$$
F_{r}(z, s)=\left\{\zeta: D^{r}(\zeta) \subset D^{s}(z)\right\} \quad \text { and } \quad G_{r}(z, s)=\bigcup_{\zeta \in D^{s}(z)} D^{r}(\zeta)
$$

By definition $F_{r}(z, s) \subset D^{s}(z) \subset G_{r}(z, s)$. Let $\gamma$ be the constant given by Lemma 1 .

Lemma 9. Let $r>0$ be fixed. There exists $c>0$ such that if $\epsilon(s)=c r / s^{\gamma^{2}}$, for all $z \in \mathbb{C}$ and $s>r$ we have

(a) $G_{r}(z, s) \subset D^{s+\epsilon(s)}(z)$.

(b) $D^{s-\epsilon(s)}(z) \subset F_{r}(z, s)$.

Proof. Applying Lemma 1 to $D^{r}(\zeta)$ and $D^{s}(z)$, and using (4), we have

$$
\frac{s^{\gamma^{2}}}{r} \lesssim \frac{s \rho(z)}{r \rho(\zeta)} \lesssim \frac{s^{1 / \gamma^{2}}}{r}
$$

(a) If $\zeta \in D^{s}(z)$ we have $s \rho(z)+r \rho(\zeta) \leq s \rho(z)\left(1+c r / s^{\gamma^{2}}\right)$ for some $c>0$.

(b) $D^{r}(\zeta) \subset D^{s}(z)$ when $|\zeta-z|+r \rho(\zeta) \leq s \rho(z)$. For $\zeta \in D^{s-\epsilon(s)}(z)$

$$
|\zeta-z|+r \rho(\zeta) \leq(s-\epsilon(s)) \rho(z)+c_{1} s \rho(z)\left(\frac{r}{s^{\gamma^{2}}}\right) .
$$

Thus if $(s-\epsilon(s)) \rho(z)+c s \rho(z)\left(r / s^{\gamma^{2}}\right) \leq s \rho(z)$ we have $D^{s-\epsilon(s)}(z) \subset F_{r}(z, s)$.

Corollary 10. Let $\left\{R_{k}\right\}_{k}$ be a partition of $\mathbb{C}$, as in Theorem 8 Define

$$
F(z, s)=\bigcup_{k: R_{k} \subset D^{s}(z)} R_{k} \quad \text { and } \quad G(z, s)=\bigcup_{k: R_{k} \cap D^{s}(z) \neq \emptyset} R_{k} .
$$

There exists a positive function $\epsilon(s)$ with $\lim _{s \rightarrow \infty} \epsilon(s) / s=0$ and such that for all $z \in \mathbb{C}$ and $s>0$

(a) $G(z, s) \subset D^{s+\epsilon(s)}(z)$.

(b) $D^{s-\epsilon(s)}(z) \subset F(z, s)$.

Proof. As the previous Lemma, using Theorem 8 (c).

We finish with a result showing that the measure of a disk cannot be too concentrated near its border.

Lemma 11. Let $\epsilon(r)$ be a positive function such that $\lim _{r \rightarrow \infty} \epsilon(r) / r=0$. Then

$$
\lim _{r \rightarrow \infty} \frac{\mu\left(D^{r+\epsilon(r)}(z)\right)}{\mu\left(D^{r}(z)\right)}=\lim _{r \rightarrow \infty} \frac{\mu\left(D^{r-\epsilon(r)}(z)\right)}{\mu\left(D^{r}(z)\right)}=1
$$

uniformly in $z \in \mathbb{C}$.

The proof is based in the following projection of the measure $\mu$. 
Lemma 12. For every $z \in \mathbb{C}$ define the measure $\nu_{z}$ on $\mathbb{R}^{+}$by

$$
\nu_{z}(A)=\mu\left(\left\{\zeta=z+r e^{i \theta}: r \in A\right\}\right) \quad A \subset \mathbb{R}^{+} .
$$

Then $\nu_{z}$ is doubling and there exists $K$ independent of $z$ such that $C_{\nu_{z}} \leq K C_{\mu}$.

Proof. Given $x \in \mathbb{R}^{+}$and $r>0$ let $I^{r}(x)=(x-r, x+r) \cap \mathbb{R}^{+}$. We want to see that

$$
\nu_{z}\left(I^{2 r}(x)\right) \leq K C_{\mu} \nu_{z}\left(I^{r}(x)\right)
$$

for all $z \in \mathbb{C}, x \in \mathbb{R}^{+}$and $r>0$.

Let $A_{z}^{r}(x)=\left\{\zeta=z+s e^{i \theta}: s \geq 0,|s-x|<r\right\}$. By definition $\nu_{z}\left(I^{2 r}(x)\right)=\mu\left(A_{z}^{2 r}(x)\right)$. Split $A_{z}^{2 r}(x)$ into $k:=\left[\frac{2 \pi}{4 r}\right]$ sectors

$$
S_{j}=\left\{\zeta=z+s e^{i \theta}: s \geq 0,|s-x|<2 r,(j-1) \frac{2 \pi}{k} \leq \theta<j \frac{2 \pi}{k}\right\} \quad j=1, \ldots, k .
$$

Being $\mu$ doubling there exists $K>0$ such that $\mu\left(S_{j}\right) \leq K C_{\mu} \mu\left(\tilde{S}_{j}\right)$, where $\tilde{S}_{j}$ is half the sector $S_{j}$, i.e.

$$
\tilde{S}_{j}=\left\{\zeta=z+s e^{i \theta}: s \geq 0,|s-x|<r,(j-1) \frac{2 \pi}{k}+\frac{2 \pi}{4 k}<\theta<j \frac{2 \pi}{k}-\frac{2 \pi}{4 k}\right\} .
$$

Since the $\tilde{S}_{j}$ 's are disjoint and $\cup_{j} \tilde{S}_{j} \subset A_{z}^{r}(x)$, we get

$$
\begin{aligned}
\nu_{z}\left(I^{2 r}\right)(x) & =\mu\left(A_{z}^{2 r}(x)\right)=\sum_{j=1}^{k} \mu\left(S_{j}\right) \leq K C_{\mu} \sum_{j=1}^{k} \mu\left(\tilde{S}_{j}\right) \leq K C_{\mu} \mu\left(A_{z}^{r}(x)\right) \\
& =K C_{\mu} \nu_{z}\left(I^{r}(x)\right) .
\end{aligned}
$$

Proof of Lemma 11. It is enough to see that

$$
\lim _{r \rightarrow \infty} \frac{\mu\left(D^{r+\epsilon(r)}(z) \backslash D^{r}(z)\right)}{\mu\left(D^{r}(z)\right)}=0
$$

uniformly in $z$. By definition of $\nu_{z}$ we have

$$
\frac{\mu\left(D^{r+\epsilon(r)}(z) \backslash D^{r}(z)\right)}{\mu\left(D^{r}(z)\right)}=\frac{\nu_{z}((r \rho(z),(r+\epsilon(r)) \rho(z))}{\nu_{z}((0, r))},
$$

and by the corresponding version of Lemma 1 for doubling measures in $\mathbb{R}^{+}$, and by Lemma 12 , there exists $K>0$ independent of $z$ such that

$$
\frac{\nu_{z}((r \rho(z),(r+\epsilon(r)) \rho(z))}{\nu_{z}((0, r))} \leq K\left(\frac{\epsilon(r)}{r}\right)^{\gamma} .
$$


Remark 4. An analogous result is true if in the definition of $\nu_{z}$ we use, instead of a radial projection with respect to $z$, a projection associated to quasi-squares of a fixed ratio $\alpha \in\left[e^{-1}, e\right]$ ( $e$ is the constant of Theorem $8(\mathrm{~b}))$. Let $Q_{\alpha}^{r}(z)$ denote the rectangle with vertices $z+r(1+i \alpha)$, $z+r(1-i \alpha), z-r(1+i \alpha)$ and $z-r(1-i \alpha)$. Given $z \in \mathbb{C}$ consider the measure $\nu_{z}$ in $\mathbb{R}$ such that

$$
\nu_{z}\left(I^{r}(x)\right)=\mu\left(Q_{\alpha}^{x+r}(z) \backslash Q_{\alpha}^{x-r}(z)\right)
$$

on any interval $I^{r}(x)$. As before, there exists $K>0$ independent of $z \in \mathbb{C}$ and $\alpha \in\left[e^{-1}, e\right]$ such that $\nu_{z}$ is doubling with $C_{\nu} \leq K C_{\mu}$. Therefore, if $R_{\alpha}^{r}(z):=Q_{\alpha}^{r \rho(z)}(z)$,

$$
\lim _{r \rightarrow \infty} \frac{\mu\left(R_{\alpha}^{r+\epsilon(r)}(z)\right)}{\mu\left(R_{\alpha}^{r}(z)\right)}=\lim _{r \rightarrow \infty} \frac{\mu\left(R_{\alpha}^{r+\epsilon(r)}(z)\right)}{\mu\left(R_{\alpha}^{r}(z)\right)}=1
$$

uniformly in $z$.

2.2. Local behaviour and regularisation of $\phi$. Let us start with a result comparing the values of $\phi$ in a disk with the value on its centre.

Lemma 13. For every $K>0$ there exists $A=A(K)>0$ such that for all $z \in \mathbb{C}$

$$
\sup _{w \in D^{K}(z)}\left|\phi(w)-\phi(z)-h_{z}(w)\right| \leq A
$$

where $h_{z}$ is a harmonic function in $D^{K}(z)$ with $h_{z}(z)=0$.

Proof. The proof is as in [OCS98, Lemma 1]. On each $D^{K}(z)$ decompose

$$
\phi(w)=\phi(z)+h_{z}(w)+\int_{D^{K}(z)}(G(w, \eta)-G(z, \eta)) \Delta \phi(\eta),
$$

where $G$ is the Green function of the disc $D^{K}(z)$ and $h_{z}$ is a harmonic function in $D^{K}(z)$ such that $h_{z}(z)=0$. By Lemma 5 (a)

$$
\sup _{z \in \mathbb{C}} \int_{D^{K}(z)} \log \frac{K \rho(z)}{|z-\eta|} \Delta \phi(\eta)<\infty
$$

and the result holds.

We have seen in the previous section that $\rho_{\phi}(z)$ is Lipschitz (see (6)). Also, because of Lemma 1, $\phi$ is Hölder continuous of some positive order on every bounded subset of $\mathbb{C}$ (see [Chr91, Lemma 2.8]). More regularity can be attained by taking a suitable weight $\psi$ equivalent to $\phi$.

Theorem 14. Let $\phi$ be subharmonic with $\Delta \phi$ doubling. There exist $\psi \in \mathcal{C}^{\infty}(\mathbb{C})$ subharmonic and $C>0$ such that $|\psi-\phi| \leq C, \Delta \psi$ is a doubling measure and $\Delta \psi \simeq 1 / \rho_{\psi}^{2} \simeq 1 / \rho_{\phi}^{2}$. Moreover $|\nabla(\Delta \psi)| \lesssim 1 / \rho_{\phi}^{3}$

Remark 5. It follows from the construction below and Lemma 9 that $\mathcal{D}_{\Delta \phi}^{+}(\Lambda)=\mathcal{D}_{\Delta \psi}^{+}(\Lambda)$ and $\mathcal{D}_{\Delta \phi}^{-}(\Lambda)=\mathcal{D}_{\Delta \psi}^{-}(\Lambda)$ for any given sequence $\Lambda$ in $\mathbb{C}$. 
Since the spaces of functions and sequences considered do not change if $\phi$ is replaced by $\psi$, from now on we will assume that $\phi \in \mathcal{C}^{\infty}(\mathbb{C}), \Delta \phi \simeq 1 / \rho^{2}$ and $|\nabla(\Delta \phi)| \lesssim 1 / \rho^{3}$.

In the proof of this result we will need to partition $\mathbb{C}$ and discretize the measure.

Lemma 15. Let $\mu$ be a positive doubling measure in $\mathbb{C}$. Fix $m \in \mathbb{N}$. There exist $k \in \mathbb{N}$ and $C>0$ such that for any partition $\left\{R_{p}\right\}_{p}$ as in Theorem 8 with $\mu\left(R_{p}\right)=$ mk there are points $\lambda_{1}^{(p)}, \ldots, \lambda_{m k}^{(p)} \in C R_{p}$ such that

(a) $\mu_{p}=\mu_{\mid R_{p}}$ and $\nu_{p}=\sum_{j=1}^{m k} \delta_{\lambda_{j}^{(p)}}$ have the same first $m$ moments.

(b) $\Lambda=\left\{\lambda_{j}^{(p)}\right\}_{p, j}$ is a $\rho$-separated sequence.

Proof. By Lemma 5 of [OC99], there exists $k \in \mathbb{N}$ such that for all measure $\mu_{p}$ supported in a rectangle $R_{p}$ with total mass $m k$, there are points $\sigma_{1}^{(p)}, \ldots \sigma_{k}^{(p)} \in R_{p}$ such that $\mu_{p}$ and $m \sum_{j=1}^{k} \delta_{\sigma_{j}^{(p)}}$ have the same first $m$ moments.

In order to get a separated sequence replace each $\sigma_{j}^{p}$ by $m$ points

$$
\gamma_{j, l}^{(p)}=\sigma_{j}^{(p)}+\rho\left(\sigma_{j}^{(p)}\right) \tau_{j}^{(p)} e^{i 2 \pi l / m}, \quad l=0, \ldots, m-1,
$$

lying on a circle around $\sigma_{j}^{(p)}$. Since for all polynomials $p$ of degree less than $m-1$

$$
m p\left(\sigma_{j}^{(p)}\right)=\sum_{l=0}^{m-1} p\left(\gamma_{j, l}^{(p)}\right),
$$

the measures $\mu_{p}$ and $\sum_{j, l} \delta_{\gamma_{j, l}^{(p)}}$ have still the same first $m$ moments. We will be done as soon as we see that the $\tau_{j}^{(p)}$ can be chosen uniformly bounded and so that $\Lambda=\left\{\gamma_{j, l}^{(p)}\right\}$ is $\rho$-separated. For this we use a Besicovitch's lemma: the family $\left\{R_{p}\right\}_{p}$ can be split in $q$ families $\left\{R_{p}^{1}\right\}_{p \in I_{1}}, \ldots\left\{R_{p}^{q}\right\}_{p \in I_{q}}$ such that two rectangles of the same family are far apart, in the sense that $M R_{p}^{l} \cap M R_{p^{\prime}}^{l}=\emptyset$, $p \neq p^{\prime}$, for some large constant $M$. For the first family $\left\{R_{p}^{1}\right\}_{p \in I_{1}}$, it is easy to choose $\tau_{j}^{(p)}$ such that the resulting sequence $\Gamma_{1}=\left\{\gamma_{j, l}^{(p)}: p \in I_{1} ; j=1, \ldots, k ; l=0, \ldots m-1\right\}$ is $\rho$-separated. Next we choose $\tau_{j}^{(p)}, p \in I_{2}$, so that $\Gamma_{2} \cap \Gamma_{1}$ is $\rho$-separated, where $\Gamma_{2}=\left\{\gamma_{j, l}^{(p)}: p \in I_{2} ; j=\right.$ $1, \ldots, k ; l=0, \ldots, m-1\}$. Choosing $\tau_{j}^{(p)}$ recursively in this way we obtain $\Lambda=\Gamma_{1} \cup \ldots \cup \Gamma_{q}$ $\rho$-separated.

Proof of Theorem 14. For any $M$ (to be chosen later) consider $k \in \mathbb{N}$ as in Lemma 15 and a partition $\left\{R_{p}\right\}_{p}$ as in Theorem 8 . Take then the sequence $\Lambda=\left\{\lambda_{j}^{(p)}\right\}_{j, p}$ given by Lemma 15 . Recall that $\lambda_{j}^{(p)} \in C R_{p}, \mu\left(R_{p}\right)=M k$ and that the measures $\mu_{p}$ and $\nu_{p}=\sum_{j=1}^{M k} \delta_{\lambda_{j}^{(p)}}$ have the same first $M$ moments. 
By Theorem 8(c) there exists $r>0$ such that $C R_{p} \subset D^{r}\left(\lambda_{j}^{(p)}\right)$ for any $p \in \mathbb{N}$ and $i \leq k$. Furthermore, by construction of $\left\{R_{p}\right\}_{p}$ there exists $q \in \mathbb{N}$ such that any $z \in \mathbb{C}$ lies in at most $q$ $\operatorname{disks} D^{r}\left(\lambda_{j}^{(p)}\right)$.

We now regularise $\nu_{p}$ by setting

$$
\tilde{\nu}_{p}=\sum_{j=1}^{M k} \frac{\mathcal{X}\left(\frac{\left|z-\lambda_{j}^{(p)}\right|}{r \rho\left(\lambda_{j}^{(p)}\right)}\right)}{\int \mathcal{X}\left(\frac{\left|z-\lambda_{j}^{(p)}\right|}{r \rho\left(\lambda_{j}^{(p)}\right)}\right)},
$$

where $\mathcal{X}$ is a smooth non-negative cut-off function of one real variable such that $\mathcal{X}(t)=1$ if $|t|<1, \mathcal{X}(t)=0$ if $|t|>2$ and $\left|\mathcal{X}^{\prime}\right|$ is bounded.

Notice that $\tilde{\nu}_{p}$ and $\mu_{p}$ have the first $M$ moments. Indeed, by the mean value property

$$
\int_{\mathbb{C}} z^{l} d \tilde{\nu}_{p}=\sum_{i=1}^{M k}\left(\lambda_{j}^{(p)}\right)^{l} \quad l=0, \ldots, M-1 .
$$

Define $\tilde{\nu}=\sum_{p=1}^{\infty} \tilde{\nu}_{p}$ and

$$
\psi(z)=\phi(z)+\frac{1}{2 \pi} \int_{\mathbb{C}} \log |z-\zeta|(\tilde{\nu}-\Delta \phi)(\zeta) .
$$

We claim that $\tilde{\nu}$ is a doubling measure. The proof of this fact is a bit technical and will be deferred to the end.

By definition $\Delta \psi=\tilde{\nu}$. Also, $\tilde{\nu}(z)$ is a sum of at most $q$ terms of order $1 / \rho^{2}\left(\lambda_{j}^{(p)}\right)$, with $z \in D^{r}\left(\lambda_{j}^{(p)}\right)$. Therefore $\Delta \psi \simeq 1 / \rho_{\phi}^{2}$ and $|\nabla(\Delta \psi)| \lesssim 1 / \rho_{\phi}^{3}$. In particular

$$
\int_{D_{\phi}(z)} \Delta \psi(\zeta) \simeq \int_{D_{\phi}(z)} \frac{d m(\zeta)}{\rho_{\phi}^{2}(\zeta)} \simeq 1
$$

hence $\rho_{\phi} \simeq \rho_{\psi}$.

Let us show next that $|\psi-\phi| \leq C$ for some $C>0$.

Let $a_{p}$ denote the centre of $R_{p}$. Assume $z \in R_{p_{0}}$ and let $I_{p_{0}}=\left\{p \in \mathbb{N}: d_{\phi}\left(a_{p}, a_{p_{0}}\right) \leq 10 r\right\}$. Remark that for $p \notin I_{p_{0}}, \zeta \in \operatorname{supp}\left(\tilde{\nu}_{p}\right)$ and $z \in \operatorname{supp}\left(\tilde{\nu}_{p_{0}}\right)$ we have $d_{\phi}(z, \zeta) \simeq d_{\phi}\left(a_{p}, a_{p_{0}}\right)$. Indeed, this follows from

$$
\left|\zeta-a_{p}\right| \leq 3 r \rho\left(a_{p}\right) \leq \frac{3}{10}\left|a_{p}-a_{p_{0}}\right|
$$

the analogous estimate for $\left|z-a_{p_{0}}\right|$ and Lemma 4 . This yields

$$
\int_{\mathbb{C}} d_{\phi}^{-M}(z, \zeta) \tilde{\nu}_{p}(\zeta) \lesssim \int_{\mathbb{C}} d_{\phi}^{-M}(z, \zeta) \mu_{p}(\zeta) \quad z \in I_{p_{0}}, p \notin I_{p_{0}} .
$$


We split

$$
2 \pi(\psi(z)-\phi(z))=\sum_{p \in I_{p_{0}}} \int_{\mathbb{C}} \log |z-\zeta|\left(\tilde{\nu}_{p}-\mu_{p}\right)(\zeta)+\sum_{p \notin I_{p_{0}}} \int_{\mathbb{C}} \log |z-\zeta|\left(\tilde{\nu}_{p}-\mu_{p}\right)(\zeta)
$$

and estimate each sum separately.

Let $p_{M}$ denote the $M$-th Taylor polynomial of $\log (|z-\zeta| / \rho(z))$. Since $\tilde{\nu}_{p}-\mu_{p}$ have vanishing moments of order less or equal to $M$, we can estimate

$$
\begin{aligned}
I_{1}: & =\left|\sum_{p \notin I_{p_{0}}} \int_{\mathbb{C}} \log \right| z-\zeta\left|\left(\tilde{\nu}_{p}-\mu_{p}\right)(\zeta)\right| \\
& =\left|\sum_{p \notin I_{p_{0}}} \int_{\mathbb{C}}\left(\log \frac{|z-\zeta|}{\rho(z)}-p_{M}(\zeta)\right)\left(\tilde{\nu}_{p}-\mu_{p}\right)(\zeta)\right| \leq \sum_{p \notin I_{p_{0}}} \int_{\mathbb{C}}\left(\frac{\rho(z)}{|z-\zeta|}\right)^{M}\left(\tilde{\nu}_{p}+\mu_{p}\right)(\zeta) .
\end{aligned}
$$

Taking $M$ big enough and using (8) and Lemmas 4(a) and 5(b),

$$
I_{1} \lesssim \int_{\mathbb{C} \backslash D^{r}(z)}\left(\frac{\rho(z)}{|z-\zeta|}\right)^{M} \mu(\zeta) \lesssim \int_{\mathbb{C} \backslash B(z, C r)} \frac{d \mu(\zeta)}{d_{\phi}^{\delta M}(z, \zeta)} \leq C
$$

For the remaining term we use again the moment condition together with the fact that for $p \in I_{p_{0}}$ there exists $\gamma$ such that $\cup\left\{\operatorname{supp}\left(\tilde{\nu}_{p}\right), p \in I_{p_{0}}\right\} \subset D_{\phi}^{\gamma}(z)$. Thus

$$
\begin{aligned}
I_{2}: & =\left|\sum_{p \in I_{p_{0}}} \int_{\mathbb{C}} \log \right| z-\zeta\left|\left(\tilde{\nu}_{p}-\mu_{p}\right)(\zeta)\right|=\left|\sum_{p \in I_{p_{0}}} \int_{\mathbb{C}} \log \left(\frac{2 \gamma \rho(z)}{|z-\zeta|}\right)\left(\tilde{\nu}_{p}-\mu_{p}\right)(\zeta)\right| \\
& \lesssim \int_{D^{\gamma}(z)} \log \left(\frac{2 \gamma \rho(z)}{|z-\zeta|}\right)(\tilde{\nu}+\mu)(\zeta) .
\end{aligned}
$$

By Lemma 5(a) this is finite.

We prove now that $\tilde{\nu}$ is doubling. We first show that it is doubling for big balls, i.e. there exist $s_{0}>0$ and a constant $C$ depending only on the doubling constant $C_{\Delta \phi}$ of $\Delta \phi$ such that for all $s>s_{0}$ we have $\tilde{\nu}\left(D^{s}(a)\right) \leq C \tilde{\nu}\left(D^{s / 2}(a)\right)$.

As in Corollary 10, define

$$
F(a, s)=\bigcup_{p: R_{p} \subset D^{s}(a)} R_{p} \quad \text { and } \quad G(a, s)=\bigcup_{p: R_{p} \cap D^{s}(a) \neq \emptyset} R_{p} .
$$

Since $\tilde{\nu}\left(R_{p}\right) \simeq \int_{R_{p}} d \sigma / \rho^{2} \simeq \mu\left(R_{p}\right)$, we see that $\tilde{\nu}(F(a, s)) \simeq \mu(F(a, s))$ and $\tilde{\nu}(G(a, s)) \simeq$ $\mu(G(a, s))$. By Corollary 10 , also $D^{s-\epsilon(s)}(a) \subset F(a, s)$ and $D^{s+\epsilon(s)}(a) \supset G(a, s)$. This and the fact that $\mu$ is doubling yield

$$
\tilde{\nu}\left(D^{s}(a)\right) \leq \tilde{\nu}(G(a, s)) \simeq \mu(G(a, s)) \leq \mu\left(D^{s+\epsilon(s)}(a)\right) \leq C_{\Delta \phi} \mu\left(D^{1 / 2(s+\epsilon(s))}(a)\right),
$$

and

$$
\tilde{\nu}\left(D^{s / 2}(a)\right) \geq \tilde{\nu}(F(a, s / 2)) \simeq \mu(F(a, s / 2)) \geq \mu\left(D^{s / 2-\epsilon(s / 2)}(a)\right) .
$$


Therefore

$$
\tilde{\nu}\left(D^{s}(a)\right) \leq C_{\Delta \phi} \tilde{\nu}\left(D^{s / 2}(a)\right) \frac{\mu\left(D^{1 / 2(s+\epsilon(s))}(a)\right)}{\mu\left(D^{s / 2-\epsilon(s / 2)}(a)\right)} .
$$

Lemma 11 shows that the quotient converges to 1 as $s$ goes to infinity uniformly in $a$, so there exists $s_{0}$ such that $\tilde{\nu}\left(D^{s}(a)\right) \leq 2 C_{\Delta \phi} \tilde{\nu}\left(D^{s / 2}(a)\right)$ for all $s \geq s_{0}$.

Corollary 3 implies that $\tilde{\nu} \simeq 1 / \rho^{2}(a)$ on $D^{s}(a)$ when $s \leq s_{0}$, so we deduce that $\tilde{\nu}\left(D^{s}(a)\right) \lesssim$ $\tilde{\nu}\left(D^{s / 2}(a)\right)$.

2.3. Generalized weighted spaces. Let $\alpha \in \mathbb{R}$ and $\phi$ be a subharmonic function whose Laplacian is a doubling measure. Let us consider the following space:

$$
\mathcal{F}_{\phi, \alpha}^{p}=\left\{f \in H(\mathbb{C}):\|f\|_{p, \alpha}^{p}=\int_{\mathbb{C}}|f|^{p} e^{-p \phi} \rho^{\alpha p-2}<\infty\right\} .
$$

Lemma 16. Let $\phi$ be a subharmonic function with a doubling Laplacian. For any $\epsilon>0$, there exists a function $\rho^{\prime}$ such that

$$
\rho_{\phi} \simeq \rho^{\prime} \quad \text { and } \quad\left|\Delta \log \rho^{\prime}\right| \leq \frac{\epsilon}{\rho_{\phi}^{2}}
$$

From this it follows that $\mathcal{F}_{\phi, \alpha}^{p}$ can be written as $\mathcal{F}_{\psi}^{p}$ for a suitable subharmonic $\psi$ with doubling Laplacian. If we have a $\phi$ already regularised by the above process, then $\psi=\phi-\alpha \log \rho^{\prime}$ will satisfy

$$
(1-\alpha \epsilon) \Delta \phi \leq \Delta \psi \leq(1+\alpha \epsilon) \Delta \phi .
$$

Therefore $\Delta \psi$ is a doubling measure. Furthermore,

$$
e^{-\psi}=e^{-\phi} \rho^{\prime \alpha} \simeq e^{-\phi} \rho^{\alpha} \text { and } \rho_{\psi} \simeq \rho_{\phi},
$$

hence $\mathcal{F}_{\psi}^{p}$ and $\mathcal{F}_{\phi, \alpha}^{p}$ are the same space.

Proof. For $r$ positive real, set $\rho_{r}:=\rho_{\phi / r}$, it follows immediately from (4) that there exists $0<$ $\varepsilon<m$ such that

$$
r^{\varepsilon} \rho_{\phi} \lesssim \rho_{r} \lesssim r^{m} \rho_{\phi}
$$

Take a sequence $\Lambda$ such that $\left\{D\left(\lambda, \rho_{r}(\lambda) / 2\right)\right\}_{\lambda \in \Lambda}$ covers $\mathbb{C}$ and the disks $\left\{D\left(\lambda, \rho_{r}(\lambda) / 10\right)\right\}_{\lambda \in \Lambda}$ are pairwise disjoint, which exists by a standard covering Lemma, see [Mat95, Theorem 2.1]. Let $\chi$ be a smooth real function with compact support in $D(0,1)$ such that $\chi=1$ on $D(0,1 / 2)$ and such that $|\nabla \chi| \leq 2,\left|\nabla^{2} \chi\right| \leq 4$. We then define

$$
\chi_{\lambda}(z)=\chi\left(\frac{|z-\lambda|}{\rho_{r}(\lambda)}\right)
$$

and $\rho_{r}^{\prime}:=\sum_{\lambda \in \Lambda} \rho_{r}(\lambda) \chi_{\lambda}$ 
Let us prove that for $r$ big enough this function has the desired properties. It is clear that $\rho_{r} \simeq \rho_{\phi}$. Moreover, there is a $C$ depending on the doubling constant of $\phi$ (but not on $r$ ) such that

$$
\begin{gathered}
C^{-1} \rho_{r} \leq \rho_{r}^{\prime} \leq C \rho_{r}, \\
\left|\nabla \rho_{r}^{\prime}(z)\right| \leq \sum_{\lambda \in \Lambda: z \in D(\lambda)} \rho_{r}(\lambda)\left|\nabla \chi_{\lambda}(z)\right| \leq C, \\
\left|\nabla^{2} \rho_{r}^{\prime}(z)\right| \leq \sum_{\lambda \in \Lambda: z \in D(\lambda)} \rho_{r}(\lambda)\left|\nabla^{2} \chi_{\lambda}(z)\right| \leq \sum_{\lambda \in \Lambda: z \in D(\lambda)} \frac{4}{\rho_{r}(\lambda)} \leq \frac{C}{\rho_{r}(z)} .
\end{gathered}
$$

We deduce from these estimates that

$$
\left|\Delta \log \rho_{r}^{\prime}\right| \leq \frac{\left|\nabla \rho_{r}\right|^{2}}{\rho_{r}^{2}}+\frac{\left|\nabla^{2} \rho_{r}\right|}{\rho} \leq \frac{C}{\rho_{r}^{2}},
$$

where $C$ does not depend on $r$. By (9), picking $r$ big enough we have the Lemma.

2.4. The multiplier. A basic tool in our approach is the use of the so-called multiplier: an entire function $g$ such that $|g| \simeq e^{\phi}$ outside a neighbourhood of the zeros of $g$.

Theorem 17. Let $\phi$ be a subharmonic function such that $\Delta \phi$ is a doubling measure. There exists an entire function $g$ such that

(a) The zero-sequence $\mathcal{Z}(g)$ of $g$ is $\rho_{\phi}$-separated and $\sup _{z \in \mathbb{C}} d_{\phi}(z, \mathcal{Z}(g))<\infty$.

(b) $|g(z)| \simeq e^{\phi(z)} d_{\phi}(z, \mathcal{Z}(g))$ for all $z \in \mathbb{C}$.

The function $g$ can be chosen so that, moreover, it vanishes on a prescribed $z_{0} \in \mathbb{C}$. We say that $g$ is a multiplier associated to $\phi$.

Proof. Take a partition $\left\{R_{p}\right\}$ of $\mathbb{C}$ with $\mu\left(R_{p}\right)=2 \pi m N$ and consider the sequence $\Lambda$ given by Lemma 15. For the sake of clarity we write $R_{p}$ instead of $C R_{p}(C$ is the constant of Lemma 15). Note that now $\left\{R_{p}\right\}_{p}$ is not a partition, although there exists a uniform constant $q$ such that all points of $\mathbb{C}$ lie in at most $q$ quasi-squares $R_{p}$. As in Lemma 15 , denote $\mu_{p}=(1 / 2 \pi) \mu_{\mid R_{p}}$ and let $\nu_{p}$ be the sum of the $\lambda \in \Lambda$ associated to $R_{p}$. Recall that $\mu_{p}$ and $\nu_{p}$ have the same first $m$ moments.

Let $g$ be a holomorphic function satisfying

$$
\log |g|=\phi-\frac{1}{2 \pi} \int_{\mathbb{C}} \log |z-\zeta|\left(\Delta \phi-2 \pi \sum_{\lambda \in \Lambda} \delta_{\lambda}\right),
$$

which exists because the Laplacian of the term at the right hand side is a sum of Dirac masses. By definition $\mathcal{Z}(g)=\Lambda$, and the previous construction ensures that (a) holds.

Let us prove (b). Assume that $z \in R_{p_{0}}$ and let $I_{p_{0}}$ denote the set of indices $p$ such that $\left(2 R_{p}\right) \cap R_{p_{0}} \neq \emptyset$. As in the previous proof, split

$$
\log |g(z)|-\phi(z)=-\int_{\mathbb{C}} \log |z-\zeta|\left(\frac{\Delta \phi}{2 \pi}-\sum_{\lambda \in \Lambda} \delta_{\lambda}\right)=S_{1}(z)+S_{2}(z),
$$


where

$$
S_{1}(z):=\sum_{p \notin I_{p_{0}}} \int_{\mathbb{C}} \log |z-\zeta|\left(\nu_{p}-\mu_{p}\right)
$$

and

$$
S_{2}(z):=\sum_{p \in I_{p_{0}}} \int_{\mathbb{C}} \log |z-\zeta|\left(\nu_{p}-\mu_{p}\right) .
$$

Again as in the proof of Theorem 14, using the Taylor expansion of $\log |z-\zeta|$ together with the moment condition one sees that $\left|S_{1}(z)\right|$ is bounded.

For the second sum notice that there exits $\gamma>0$ such that $\cup_{p \in I_{p_{0}}} R_{p} \subset D^{\gamma}(z)$. Hence, denoting $|z-\Lambda|=\inf _{\lambda \in \Lambda}|z-\lambda|$, we get

$$
\begin{aligned}
S_{2}(z) & =\sum_{p \in I_{p_{0}}} \int_{\mathbb{C}} \log \frac{2 \gamma \rho(z)}{|z-\zeta|}\left(\mu_{p}-\nu_{p}\right) \leq \int_{D^{\gamma}(z)} \log \frac{2 \gamma \rho(z)}{|z-\zeta|} d \mu(\zeta)-\log \frac{2 \gamma \rho(z)}{|z-\Lambda|} \\
& \leq C_{2}+\log \frac{|z-\Lambda|}{\rho(z)} .
\end{aligned}
$$

On the other hand, using the $\rho$-separation of $\Lambda$

$$
-S_{2}(z) \leq \sum_{p \in I_{p_{0}}} \sum_{\lambda \in R_{p}} \log \frac{2 \gamma \rho(z)}{|z-\lambda|} \leq \log \frac{\rho(z)}{|z-\Lambda|}+C(\delta) \cdot \#\left(\Lambda \cap \cup_{p \in I_{p_{0}}} R_{p}\right) .
$$

Since $\# I_{p_{0}}$ is uniformly bounded, this and the estimate of $S_{1}$ give:

$$
\log \frac{|z-\Lambda|}{\rho(z)}-C \leq \log |g(z)|-\phi(z) \leq \log \frac{|z-\Lambda|}{\rho(z)}+C^{\prime}
$$

The result is then immediate from Lemma 4(a).

Next we state a useful application of the multiplier, a result about existence of peak functions. These functions attain value 1 at a given point and decay very fast away from the point. They are very useful in the estimates of the Bergman kernel and in the construction of solutions to the $\bar{\partial}$ equation. Another proof of the following Lemma, using estimates for the $\bar{\partial}$-equation, can be found in an Appendix. This second proof is along the lines of [FS89, Theorem 2.1], where a related result is proved.

Theorem 18. Take $\varepsilon>0$ and $m \in \mathbb{N}$. There exists $C>0$ such that for all $\eta \in \mathbb{C}$ there is an entire function $P_{\eta}$ with $P_{\eta}(\eta)=1$ and

$$
\left|P_{\eta}(z)\right| \leq C e^{\varepsilon(\phi(z)-\phi(\eta))} \frac{1}{1+d_{\phi}^{m}(z, \eta)} .
$$

Proof. Let $h$ be a multiplier for $\varepsilon \phi$ (constructed as in Theorem 17) with zero sequence $\Sigma=\left\{\sigma_{k}\right\}_{k}$ and such that $\{\eta\} \cup \Sigma$ is $\rho$-separated. In particular $|h(z)| \simeq e^{\varepsilon \phi(z)} d_{\phi}(z, \Sigma)$. It follows from the 
construction of the multiplier that for each $M \in \mathbb{N}$ there exists $r>0$ such that $\#(\Sigma \cap B(\lambda, r)) \gtrsim$ $M$ for all $\lambda \in \mathbb{C}$. Given $\sigma_{1}, \ldots, \sigma_{M} \in \Sigma \cap B(\eta, r)$ define

$$
P_{\eta}(z)=c_{\eta} \frac{h(z)}{\left(z-\sigma_{1}\right) \cdots\left(z-\sigma_{M}\right)} \frac{\rho^{M}(\eta)}{e^{\varepsilon \phi(\eta)}},
$$

where $c_{\eta}$ is chosen so that $P_{\eta}(\eta)=1$.

Let us observe first that there exists $c>0$ independent of $\eta$ with $c^{-1} \leq c_{\eta} \leq c$. Since $\left|\eta-\sigma_{i}\right| \simeq \rho(\eta)$, then

$$
\frac{1}{c_{\eta}}=\frac{h(\eta)}{\left(\eta-\sigma_{1}\right) \cdots\left(\eta-\sigma_{M}\right)} \frac{\rho^{M}(\eta)}{e^{\varepsilon \phi(\eta)}} \simeq \frac{e^{\varepsilon \phi(\eta)} d_{\phi}(\eta, \Sigma)}{\rho^{M}(\eta)} \frac{\rho^{M}(\eta)}{e^{\varepsilon \phi(\eta)}}=d_{\phi}(\eta, \Sigma) \simeq 1 .
$$

We split the estimate of $\left|P_{\eta}(z)\right|$ into several regions. Let $\varepsilon>0$ be such that that the balls $B\left(\sigma_{i}, \varepsilon\right)$ and $B(\eta, \varepsilon)$ are pairwise disjoint. Consider $K>0$ with $\cup_{i=1}^{M} B\left(\sigma_{i}, \varepsilon\right) \subset B(\eta, K)$.

i) $z \in \bigcup_{i=1}^{M} B\left(\sigma_{i}, \varepsilon\right)$. For $z \in B\left(\sigma_{i}, \varepsilon\right)$ we have $\rho(z) \simeq \rho(\eta) \simeq \rho\left(\sigma_{i}\right), d_{\phi}(z, \Sigma) \simeq \mid z-$ $\sigma_{i} \mid / \rho\left(\sigma_{i}\right)$ and $d_{\phi}\left(z, \sigma_{j}\right) \gtrsim 1, j \neq i$. Thus

$$
\left|P_{\eta}(z)\right| \lesssim\left|\frac{h(z)}{z-\sigma_{i}}\right| \rho(\eta) e^{-\varepsilon \phi(\eta)} \simeq e^{\varepsilon(\phi(z)-\phi(\eta))} .
$$

ii) $z \in B(\eta, K) \backslash \bigcup_{i=1}^{M} B\left(\sigma_{i}, \varepsilon\right)$. Here $\rho(z) \simeq \rho(\eta)$ and $\left|z-\sigma_{i}\right| \gtrsim \rho(\eta)$, so

$$
\left|P_{\eta}(z)\right| \lesssim \frac{e^{\varepsilon \phi(z)} d_{\phi}(z, \Sigma)}{\rho^{M}(\eta)} \frac{\rho^{M}(\eta)}{e^{\varepsilon \phi(\eta)}} \lesssim e^{\varepsilon(\phi(z)-\phi(\eta))}
$$

iii) $z \notin B(\eta, K)$. Here $d_{\phi}\left(z, \sigma_{i}\right) \simeq d_{\phi}(z, \eta)$, so

$$
\left|P_{\eta}(z)\right| \lesssim \frac{e^{\varepsilon \phi(z)} d_{\phi}(z, \Sigma)}{|z-\eta|^{M}} \frac{\rho^{M}(\eta)}{e^{\varepsilon \phi(\eta)}} \lesssim e^{\varepsilon(\phi(z)-\phi(\eta))}\left(\frac{\rho(\eta)}{|z-\eta|}\right)^{M} .
$$

This and Lemma 4(b) give the result.

\section{BASIC PROPERTIES OF FUNCTIONS IN $\mathcal{F}_{\phi}^{p}$}

Here we study the behaviour of functions in $\mathcal{F}_{\phi}^{p}$ and related topics. We prove the estimates with norms $\|\cdot\|_{\mathcal{F}_{\phi}^{p}}$ on the solutions to the $\bar{\partial}$ equation (Theorem $\mathrm{C}$ ) and provide estimates of the Bergman Kernel of $\mathcal{F}_{\phi}^{2}$ on the diagonal. We also introduce a scaled translation in the plane that gives rise to a translated weight and to an isometry between the spaces of functions for the original and the translated weight. This will be used when studying the properties of weak limits (Section 3.5). 
3.1. Pointwise estimates. Let us first see what is the natural growth of functions in $\mathcal{F}_{\phi}^{p}$. Recall that $d \sigma=d m / \rho^{2}$.

Lemma 19. Let $1 \leq p<\infty$. For any $r>0$ there exists $C=C(r)>0$ such that for any $f \in H(\mathbb{C})$ and $z \in \mathbb{C}$ :

(a) $|f(z)|^{p} e^{-p \phi(z)} \leq C \int_{D^{r}(z)}|f|^{p} e^{-p \phi} d \sigma$.

(b) $\left|\nabla\left(|f| e^{-\phi}\right)(z)\right| \leq C\left(\int_{D^{r}(z)}|f|^{p} e^{-p \phi} d \sigma\right)^{1 / p}$.

(c) If $s>r$ then $|f(z)|^{p} e^{-p \phi(z)} \leq C_{s} \int_{D^{s}(z) \backslash D^{r}(z)}|f|^{p} e^{-p \phi} d \sigma$.

Proof. Let $H_{z}$ be a holomorphic function with $R e H_{z}=h_{z}$, where $h_{z}$ is the harmonic function in $D^{r}(z)$ given in Lemma 13 .

(a) is proved as in [OCS98, Lemma 1]:

$$
\begin{aligned}
|f(z)|^{p} e^{-p \phi(z)} & =\left|f(z) e^{-H_{z}(z)}\right|^{p} e^{-p \phi(z)} \\
& \leq \frac{C}{\rho^{2}(z)} \int_{D^{r}(z)}|f(\zeta)|^{p} e^{-p\left(h_{z}(\zeta)+\phi(z)\right)} \simeq \int_{D^{r}(z)}|f|^{p} e^{-p \phi} d \sigma .
\end{aligned}
$$

(b) First let us see that $\left|\partial \phi / \partial \zeta-\partial h_{z} / \partial \zeta\right| \lesssim 1$ on $D^{r}(z)$. By (7), if $\zeta \in D^{r}(z)$

$$
\left|\frac{\partial \phi}{\partial \zeta}(\zeta)-\frac{\partial h_{z}}{\partial \zeta}(\zeta)\right|=\left|\frac{\partial}{\partial \zeta} \int_{D^{r}(z)} G(\zeta, \eta) \Delta \phi(\eta)\right| \leq \int_{D^{r}(z)} \frac{2 r \rho(z)}{|\zeta-\eta|} \Delta \phi(\eta) .
$$

Take $s$ (depending on $r$ ) such that $D^{r}(z) \subset D^{s}(\zeta)$. From $\Delta \phi \simeq 1 / \rho^{2}$ we deduce

$$
\int_{D^{r}(z)} \frac{2 r \rho(z)}{|\zeta-\eta|} \Delta \phi(\eta) \lesssim \frac{1}{\rho(\zeta)} \int_{D^{s}(\zeta)} \frac{d m(\eta)}{|\zeta-\eta|} \simeq 1
$$

Since $\left|\nabla\left(|f| e^{-\phi}\right)\right|=\left|f^{\prime}-2 f \partial \phi / \partial z\right| e^{-\phi}$, we have

$$
\left|\nabla\left(f e^{-H_{z}}\right)(z)\right|=\left|f^{\prime}(z)-2 f(z) h_{z}^{\prime}(z)\right| \simeq\left|\nabla\left(|f| e^{-\phi}\right)(z)\right| e^{\phi(z)} .
$$

On the other hand,

$$
\left|\nabla\left(f e^{-H_{z}}\right)(z)\right| \lesssim\left|\int_{|z-\zeta|=\rho(z)} \frac{f(\zeta) e^{-H_{z}(\zeta)}}{(z-\zeta)^{2}} d \zeta\right| \simeq \frac{1}{\rho^{2}(z)} \int_{|z-\zeta|=\rho(z)}|f(\zeta)| e^{-h_{z}(\zeta)}|d \zeta| .
$$

From (a), for $|z-\zeta|=\rho(z)$

$$
|f(\zeta)| e^{-\phi(\zeta)} \lesssim\left(\int_{D^{r}(z)}|f|^{p} e^{-p \phi} d \sigma\right)^{1 / p}
$$

By Lemma 13 we have then

$$
\left|\nabla\left(f e^{-H_{z}}\right)(z)\right| \lesssim \frac{1}{\rho(z)}\left(\int_{D^{r}(z)}|f|^{p} e^{-p \phi} d \sigma\right)^{1 / p} e^{\phi(z)},
$$

which together with $(10)$ concludes the proof. 
(c) As (a), using the subharmonicity of $\left|f e^{-H_{z}}\right|^{p}$.

Lemma 20. Let $1 \leq p<\infty$. For any entire function $g$ with $g(\lambda)=0$ we have

$$
\left|g^{\prime}(\lambda)\right| e^{-\phi(\lambda)} \lesssim \frac{1}{\rho(\lambda)}\left(\int_{D(\lambda)}|g|^{p} e^{-p \phi} d \sigma\right)^{1 / p}
$$

Proof. Lemma 19.(c) with $r=1 / 2$ and $s=1$ applied to the function $g(z) /(z-\lambda)$ yields

$$
\left|g^{\prime}(\lambda)\right|^{p} e^{-p \phi(\lambda)} \lesssim \int_{D(\lambda) \backslash D^{1 / 2}(\lambda)} \frac{|g(z)|^{p}}{|z-\lambda|^{p}} e^{-p \phi(z)} d \sigma \lesssim \frac{1}{\rho^{p}(\lambda)} \int_{D(\lambda)}|g(z)|^{p} e^{-p \phi(z)} d \sigma .
$$

3.2. Hörmander type estimates. This section is devoted to the proof of the $\bar{\partial}$-estimates of Theorem $\mathrm{C}$ in the introduction.

Theorem C. Let $\phi$ be a subharmonic function such that $\Delta \phi$ is a doubling measure. There is a solution $u$ to the equation $\bar{\partial} u=f$ such that $\left\|u e^{-\phi}\right\|_{L^{p}(\mathbb{C})} \lesssim\left\|f e^{-\phi} \rho\right\|_{L^{p}(\mathbb{C})}$ for any $1 \leq p \leq \infty$.

Proof. Take $\varepsilon=1$ and consider the peak-functions given by Theorem 18 , By Lemma 19(b), there exists $r>0$ such that $\left|P_{\eta}(z)\right| \gtrsim e^{\phi(z)-\phi(\eta)}$ on $D^{r}(\eta)$, for all $\eta \in \mathbb{C}$. Take a sequence $\Lambda$ such that $\left\{D^{r}(\lambda)\right\}_{\lambda \in \Lambda}$ covers $\mathbb{C}$ and the disks $\left\{D^{r / 5}(\lambda)\right\}_{\lambda \in \Lambda}$ are pairwise disjoint, which exist by a standard covering Lemma, see [Mat95, Theorem 2.1]. Let $\left\{\chi_{\lambda}\right\} \subset \mathcal{C}_{0}^{\infty}$ be a partition of unity associated to $\left\{D^{r}(\lambda)\right\}_{\lambda}$.

Decompose the datum $f=\sum f_{\lambda}$, with $f_{\lambda}(z)=f(z) \chi_{\lambda}(z)$. By Theorem 18 , for any $\lambda$ there exists an entire function $m_{\lambda}(z)=P_{\lambda}(z) e^{-\phi(\lambda)}$ such that

$$
\left|m_{\lambda}(z)\right| \lesssim e^{\phi(z)} \frac{1}{d_{\phi}^{M}(z, \lambda)+1}
$$

The radius $r$ has been chosen so that $\left|m_{\lambda}(\zeta)\right| \gtrsim e^{\phi(\zeta)}$ if $\zeta \in D^{r}(\lambda)$. Define

$$
u_{\lambda}(z)=m_{\lambda}(z) \frac{1}{\pi} \int_{D^{r}(\lambda)} \frac{f_{\lambda}(\zeta) / m_{\lambda}(\zeta)}{\zeta-z} d m(\zeta) .
$$

Clearly $\bar{\partial} u_{\lambda}=f_{\lambda}$, thus $u=\sum_{\lambda \in \Lambda} u_{\lambda}$ is as a solution to $\bar{\partial} u=f$. We must prove the size estimates. As we have used a linear operator to construct $u$ from the datum $f$, we only need to check that $\left\|u e^{-\phi}\right\|_{L^{\infty}} \lesssim\left\|f e^{-\phi} \rho\right\|_{L^{\infty}}$ and $\left\|u e^{-\phi}\right\|_{L^{1}} \lesssim\left\|f e^{-\phi} \rho\right\|_{L^{1}}$. The estimates for $1<p<\infty$ follow then by Marcinkiewicz interpolation theorem.

Assume that $z \in D^{r}(\lambda)$ and take $K>0$ such that $D^{r}(z) \subset D^{K}(\lambda)$. Then

$$
\left|u_{\lambda}(z) e^{-\phi(z)}\right| \lesssim \int_{D^{r}(z)} \frac{|f(\zeta)| e^{-\phi(\zeta)} \rho(\zeta)}{\rho(z)|\zeta-z|} d m(\zeta) \lesssim \int_{D^{K}(\lambda)} \frac{|f(\zeta)| e^{-\phi(\zeta)} \rho(\zeta)}{\rho(\lambda)|\zeta-z|} d m(\zeta)
$$


On the other hand, if $z \notin D^{r}(\lambda)$

$$
\begin{aligned}
\left|u_{\lambda}(z) e^{-\phi(z)}\right| & \lesssim d_{\phi}^{-M}(z, \lambda) \int_{D^{r}(\lambda)} \frac{|f(\zeta)| e^{-\phi(\zeta)}}{|\zeta-z|} d m(\zeta) \\
& \lesssim \frac{d_{\phi}^{-M}(z, \lambda)}{\rho^{2}(\lambda)} \int_{D^{r}(\lambda)}|f(\zeta)| e^{-\phi(\zeta)} \rho(\zeta) d m(\zeta)
\end{aligned}
$$

Therefore, applying Lemma 6

$$
\begin{aligned}
\left\|u e^{-\phi}\right\|_{L^{\infty}} & \lesssim\left\|f e^{-\phi} \rho\right\|_{L^{\infty}} \sup _{z \in \mathbb{C}}\left(\int_{D^{r}(z)} \frac{d m(\zeta)}{\rho(z)|z-\zeta|}+\sum_{\lambda: z \notin D^{r}(\lambda)} d_{\phi}^{-M}(z, \lambda)\right) \\
& \lesssim\left\|f e^{-\phi} \rho\right\|_{L^{\infty}} .
\end{aligned}
$$

In the $L^{1}$ norm we get

$$
\begin{aligned}
\left\|u e^{-\phi}\right\|_{L^{1}} \lesssim \sum_{\lambda \in \Lambda} & \left(\int_{z \in D^{r}(\lambda)} \int_{\zeta \in D^{K}(\lambda)} \frac{|f(\zeta)| e^{-\phi(\zeta)} \rho(\zeta)}{\rho(\lambda)|\zeta-z|} d m(\zeta) d m(z)+\right. \\
& \left.\int_{z \notin D^{r}(\lambda)} \frac{d_{\phi}^{-M}(z, \lambda)}{\rho(\lambda)^{2}} \int_{D^{r}(\lambda)}|f(\zeta)| e^{-\phi(\zeta)} \rho(\zeta) d m(\zeta) d m(z)\right) .
\end{aligned}
$$

Reversing the order of integration we immediately get $\left\|u e^{-\phi}\right\|_{L^{1}} \lesssim\left\|f e^{-\phi} \rho\right\|_{L^{1}}$.

3.3. Bergman kernel estimates. Let $K_{\phi}(z, \zeta)$ denote the Bergman kernel for $\mathcal{F}_{\phi}^{2}$, i.e, for any $f \in \mathcal{F}_{\phi}^{2}$

By definition

$$
f(z)=\left\langle K_{\phi}(z, \cdot), f\right\rangle=\int_{\mathbb{C}} K_{\phi}(z, \zeta) f(\zeta) e^{-2 \phi(\zeta)} d \sigma(\zeta)
$$

$$
K_{\phi}(z, z)=\int_{\mathbb{C}}\left|K_{\phi}(z, \zeta)\right|^{2} e^{-2 \phi(\zeta)} d \sigma(\zeta) .
$$

Lemma 21. There exists $C>0$ such that

$$
C^{-1} e^{2 \phi(z)} \leq K_{\phi}(z, z) \leq C e^{2 \phi(z)} \quad z \in \mathbb{C} .
$$

Proof. We use the identity

$$
\sqrt{K_{\phi}(z, z)}=\sup \left\{|f(z)|: f \in \mathcal{F}_{\phi}^{2},\|f\|_{\mathcal{F}_{\phi}^{2}} \leq 1\right\} .
$$

The estimate $\sqrt{K_{\phi}(z, z)} \lesssim e^{\phi(z)}$ is immediate from Lemma 19 (a). In order to prove the reverse estimate we construct $f \in \mathcal{F}_{\phi}^{2}$ with $\|f\|_{\mathcal{F}_{\phi}^{2}} \leq 1$ and $|f(z)| \geq C e^{\phi(z)}$, for some constant $C$ independent of $z$.

By Theorem 18 , for every $m \in \mathbb{N}$ there exists $P_{z}$ entire such that

$$
\left|P_{z}(\zeta)\right| \leq C e^{\phi(\zeta)-\phi(z)} \frac{1}{1+d_{\phi}^{m}(z, \zeta)},
$$


with $C$ independent of $z$. Define $f_{z}(\zeta)=c_{0} e^{\phi(z)} P_{z}(\zeta)$, where $c_{0}$ is a positive constant to be chosen later. Now $f_{z}(z)=c_{0} e^{\phi(z)}$ and

$$
\left|f_{z}(\zeta)\right|^{2} e^{-2 \phi(\zeta)} \rho^{-2}(\zeta) \leq \frac{c_{0} C}{1+d_{\phi}^{2 m}(z, \zeta)} \Delta \phi(\zeta),
$$

hence by Lemma $5(\mathrm{~b})$ there exist $c_{0}$ and $C$ independent of $z$ so that $\left\|f_{z}\right\|_{\mathcal{F}_{\phi}^{2}} \leq 1$.

Remark 6. This argument and Lemma 19 (a) show that for any $p \in[1, \infty]$,

$$
\sup \left\{|f(z)|: f \in \mathcal{F}_{\phi}^{p},\|f\|_{\mathcal{F}_{\phi}^{p}} \leq 1\right\} \simeq e^{\phi(z)} .
$$

3.4. Scaled translations and invariance. In this section we introduce the scaled translation and its main properties.

Given $\phi$ consider the class $W_{\phi}$ of subharmonic functions $\psi$ such that

(i) $\Delta \psi$ doubling with $C_{\Delta \psi} \leq C_{\Delta \phi}$.

(ii) $\int_{D_{\phi}(0)} \Delta \psi \simeq 1$.

(iii) $\psi(0)=0$.

An important property of $W_{\phi}$ is that there exists $\eta$ such that $\Delta \psi(z) \lesssim|z|^{2 \eta}$ for all $\psi \in W_{\phi}$ of class $\mathcal{C}^{2}$. This is a consequence of (5) and the fact that $\Delta \psi \simeq 1 / \rho_{\psi}^{2}$.

Fix $q>2 \eta+1$ and consider the kernel

$$
\kappa(z, \zeta):=\frac{1}{2 \pi}\left[\log \left|1-\frac{z}{\zeta}\right|-\operatorname{Re}\left(P_{q}\left(\frac{z}{\zeta}\right)\right) \chi_{\mathbb{C} \backslash D(0,1)}(\zeta)\right],
$$

where $P_{q}$ is the Taylor polynomial of degree $q$ of $\log (1+x)$ around $x=0$, and its associated integral operator

$$
K[f](z)=\int_{\mathbb{C}} \kappa(z, \zeta) f(\zeta) d m(\zeta) .
$$

This operator solves the Poisson equation, that is $\Delta K[f]=f$.

For every $x \in \mathbb{C}$, consider the scaled translation

$$
\tau_{x}(z)=x+z \rho_{\phi}(x),
$$

the associated subharmonic function

$$
\phi_{x}(z)=K\left[\Delta\left(\phi \circ \tau_{x}\right)\right](z)-K\left[\Delta\left(\phi \circ \tau_{x}\right)\right](0) .
$$

Define also $\mathrm{h}_{x}:=\phi \circ \tau_{x}-\phi_{x}$. It is clear that $\mathrm{h}_{x}$ is harmonic. Take then $H_{x}$ holomorphic having $\mathrm{h}_{x}$ as real part and consider the scaled translation operator

$$
T_{x}^{\phi} f(z)=f\left(\tau_{x}(z)\right) e^{-H_{x}(z)} .
$$

Lemma 22. For every $x \in \mathbb{C}$,

(a) The subharmonic function $\phi_{x}$ belongs to $W_{\phi}$ and $\rho_{\phi_{x}}(0)=1$.

(b) $T_{x}^{\phi}$ is an isometry from $\mathcal{F}_{\phi}^{p}$ to $\mathcal{F}_{\phi_{x}}^{p}$, for $1 \leq p \leq \infty$. 
Proof. Note first that from the identity

$$
1=\int_{D_{\phi_{x}}(z)} \Delta \phi_{x}=\int_{D_{\phi_{x}}(z)} \rho_{\phi}^{2}(x) \Delta \phi\left(\tau_{x}(\zeta)\right)=\int_{D\left(\tau_{x}(z), \rho_{\phi_{x}}(z) \rho_{\phi}(x)\right)} \Delta \phi
$$

it follows that

$$
\rho_{\phi}\left(\tau_{x}(z)\right)=\rho_{\phi_{x}}(z) \rho_{\phi}(x) .
$$

This implies that the mapping $\tau_{x}$ is actually an isometry between $\mathbb{C}$ endowed with the distance $d_{\phi_{x}}$ and $\mathbb{C}$ with $d_{\phi}$, that is

$$
d_{\phi_{x}}(z, \zeta)=d_{\phi}\left(\tau_{x}(z), \tau_{x}(\zeta)\right) \quad \forall z, \zeta \in \mathbb{C} .
$$

(a) By definition $\phi_{x}(0)=0$, and by (11), $\rho_{\phi_{x}}(0)=1$. This gives properties (ii) and (iii) of $W_{\phi}$. It is also clear that $\Delta \phi_{x}$ is doubling and $C_{\Delta \phi_{x}}=C_{\Delta \phi}$, since for any $a \in \mathbb{C}$ and $r>0$ :

$$
\int_{D(a, 2 r)} \Delta \phi_{x}=\int_{D\left(\tau_{x}(a), 2 r \rho_{\phi}(x)\right)} \Delta \phi \leq C_{\Delta \phi} \int_{D\left(\tau_{x}(a), r \rho_{\phi}(x)\right)} \Delta \phi \leq C_{\Delta \phi} \int_{D(a, r)} \Delta \phi_{x} .
$$

(b) For $p<\infty$ we use the change of variable $\zeta=\tau_{x}(z)$ and (11):

$$
\begin{aligned}
\int_{\mathbb{C}}\left|T_{x}^{\phi}(f)(z)\right|^{p} e^{-p \phi_{x}(z)} \rho_{\phi_{x}}^{-2}(z) d m(z) & =\int_{\mathbb{C}}\left|f\left(\tau_{x}(z)\right)\right|^{p} e^{-p \phi\left(\tau_{x}(z)\right)}\left(\frac{\rho_{\phi}\left(\tau_{x}(z)\right)}{\rho_{\phi}(x)}\right)^{-2} d m(z) \\
& =\int_{\mathbb{C}}|f(\zeta)|^{p} e^{-p \phi(\zeta)} \rho_{\phi}^{-2}(\zeta) d m(\zeta) .
\end{aligned}
$$

The case $p=\infty$ is straightforward from 111 .

Given a sequence $\Lambda$ and $x \in \mathbb{C}$ let

$$
\Lambda_{x}:=\left(\tau_{x}\right)^{-1}(\Lambda)
$$

Given a sequence $\Lambda$ and $z \in \mathbb{C}$, denote $n_{\Lambda}(z, r)=\#(\Lambda \cap \overline{D(z, r)})$, for any $r>0$.

Lemma 23. Let $\Lambda$ be a sequence in $\mathbb{C}$.

(a) $\Lambda$ is $\rho$-separated if and only if $\Lambda_{x}$ is $\rho_{\phi_{x}}$-separated.

(b) $\Lambda \in \operatorname{Int} \mathcal{F}_{\phi}^{p}$ if and only if $\Lambda_{x} \in \operatorname{Int} \mathcal{F}_{\phi_{x}}^{p}$. Similarly, $\Lambda \in \operatorname{Samp} \mathcal{F}_{\phi}^{p}$ if and only if $\Lambda_{x} \in$ Samp $\mathcal{F}_{\phi_{x}}^{p}$. Furthermore, the interpolation and sampling constants remain the same.

(c) The densities are stable: $\mathcal{D}_{\Delta \phi}^{+}(\Lambda)=\mathcal{D}_{\Delta \phi_{x}}^{+}\left(\Lambda_{x}\right)$, and $\mathcal{D}_{\Delta \phi}^{-}(\Lambda)=\mathcal{D}_{\Delta \phi_{x}}^{-}\left(\Lambda_{x}\right)$.

Proof. (a) is an immediate consequence of (11).

(b) is a consequence of Lemma 22 and the identity $\left\|f\left|\Lambda\left\|_{\ell_{\phi}^{p}(\Lambda)}=\right\| T_{x}^{\phi} f\right| \Lambda_{x}\right\|_{\ell_{\phi_{x}}^{p}\left(\Lambda_{x}\right)}$.

(c) Define

$$
\mathcal{D}_{\Delta \phi}(z, r, \Lambda)=\frac{n_{\Lambda}(z, r \rho(z))}{\int_{D_{\phi}^{r}(z)} \Delta \phi}
$$


By a change of variables, it is clear that

$$
\mathcal{D}_{\Delta \phi}(z, r, \Lambda)=\mathcal{D}_{\Delta \phi_{x}}\left(\left(\tau_{x}\right)^{-1}(z), r, \Lambda_{x}\right) .
$$

Taking the supremum over $z \in \mathbb{C}$ and passing to the limsup we get the result for the upper density. The lower density is dealt with similarly.

3.5. Weak limits. In this section we study weak limits of sequences $\Lambda$ and their properties.

Definition 6. A sequence of closed sets $Q_{j}$ converges strongly to $Q$, denoted $Q_{j} \rightarrow Q$ if $\left[Q, Q_{j}\right] \rightarrow 0$; here $[Q, R]$ denotes the Fréchet distance between $Q$ and $R$. We say that $Q_{j}$ converges compactwise to $Q$, denoted $Q_{j} \rightarrow Q$, if for every compact set $K$ we have $\left(Q_{j} \cap K\right) \cup \partial K \rightarrow$ $(Q \cap K) \cup \partial K$.

Definition 7. A set $\Lambda^{*}$ is a weak limit of $\Lambda$ if there exists a sequence $\left\{x_{n}\right\}_{n \in \mathbb{N}}$ in $\mathbb{C}$ such that $\Lambda_{x_{n}} \rightarrow \Lambda^{*}$.

Given a $\rho$-separated sequence $\Lambda$, and a sequence $\left\{x_{n}\right\}_{n \in \mathbb{N}}$ it is always possible to extract a subsequence of $\Lambda_{x_{n_{j}}}$ such that $\Lambda_{x_{n_{j}}} \rightarrow \Lambda^{*}$ for some $\Lambda^{*}$. We need also a normal family argument for the translated weights that define the space.

Lemma 24. Let $\left\{x_{n}\right\}_{n \in \mathbb{N}}$ be a sequence in $\mathbb{C}$. There exist a subharmonic function $\phi^{*}$ and a subsequence $\left\{x_{n_{k}}\right\}_{k}$ such that $\left\{\phi_{x_{n_{k}}}\right\}_{k}$ and $\left\{\Delta \phi_{x_{n_{k}}}\right\}_{k}$ converge uniformly on compact sets to $\phi^{*}$ and $\Delta \phi^{*}$ respectively. Furthermore, $\Delta \phi^{*}$ is a doubling measure and $C_{\Delta \phi^{*}} \leq C_{\Delta \phi}$.

Proof. Take $\eta$ and $q>2 \eta+1$ as in the definition of the kernel $\kappa$ (see previous section). Denote $\mu_{n}=\Delta \phi_{x_{n}}$.

Since $\left|\nabla \mu_{n}\right| \lesssim \rho_{\phi_{x_{n}}}^{-3}$ (Theorem 14) and $\rho_{\phi_{x_{n}}}(0)=1$, for any compact set $K$ there exits $C_{K}>0$ such that $\left|\nabla \mu_{n}(z)\right| \leq C_{K}$. By the Arzelà-Ascoli theorem, we can extract a subsequence $\left\{\mu_{n_{k}}\right\}_{k}$ converging uniformly on compact sets of $\mathbb{C}$ to a function $\mu^{*}$. It follows immediately that the measure with density $\mu^{*}$ is doubling and $C_{\mu^{*}} \leq C_{\mu_{n}}=C_{\Delta \phi}$. Furthermore, this implies that $\rho_{\phi_{x_{n}}} \rightarrow \rho^{*}$ uniformly on compacts.

Let now $\phi^{*}=K\left[\mu^{*}\right]-K\left[\mu^{*}\right](0)$, and denote $\phi_{k}:=\phi_{x_{n_{k}}}, \mu_{k}:=\mu_{n_{k}}$. We will show that $\left\{\phi_{k}\right\}_{k}$ converges uniformly on compact sets to $\phi^{*}$.

By definition $\phi_{p}(z)=K\left[\mu_{p}\right](z)-K\left[\mu_{p}\right](0)$, thus we only have to prove that $K\left[\mu_{p}\right]$ converges uniformly on compacts set to $K\left[\mu^{*}\right]$. Take $z \in D(0, R)$ and $t>R$. Then

$$
\begin{aligned}
\left|K\left[\mu_{p}\right](z)-K\left[\mu^{*}\right](z)\right| \leq \mid \int_{\mathbb{C} \backslash D(0, t)} \kappa(z, \zeta)\left(\mu_{p}(\zeta)\right. & \left.-\mu^{*}(\zeta)\right) d m(\zeta) \mid \\
& +\left|\int_{D(0, t)} \kappa(z, \zeta)\left(\mu_{p}(\zeta)-\mu^{*}(\zeta)\right) d m(\zeta)\right|
\end{aligned}
$$

Let $I_{1}$ be the first integral. By construction of $\kappa$ we have

$$
|\kappa(z, \zeta)| \lesssim\left(\frac{R}{|\zeta|}\right)^{q} .
$$


Also, $\left(\left|\mu_{p}(\zeta)\right|+\left|\mu^{*}(\zeta)\right|\right) d m(\zeta)$ is a doubling measure with doubling constant less than $C_{\Delta \phi}$. By (5) $\left|\mu_{p}(\zeta)\right|+\left|\mu^{*}(\zeta)\right| \lesssim|\zeta|^{2 \eta}$, and therefore

$$
I_{1} \lesssim \int_{|\zeta|>t}\left(\frac{R}{|\zeta|}\right)^{q}|\zeta|^{2 \eta} d m(\zeta) .
$$

This is smaller than $\varepsilon$ for $t$ big enough.

Let $I_{2}$ be the second integral in the estimate above. We have

$$
I_{2} \lesssim \int_{D(0,1)}|\log | \frac{z-\zeta}{\zeta}||\left|\mu_{p}(\zeta)-\mu^{*}(\zeta)\right| d m(\zeta)+\int_{D(0, t) \backslash D(0,1)}\left|P_{q}\left(\frac{z}{\zeta}\right) \| \mu_{p}(\zeta)-\mu^{*}(\zeta)\right| d m(\zeta)
$$

For all $z \in D(0, R)$ and $\zeta \in D(0, t) \backslash D(0,1)$ we have $\left|P_{q}(z / \zeta)\right| \leq C(R, t)$, hence the uniform convergence of $\mu_{p}$ implies that for $p$ big enough the second integral here is smaller than $\varepsilon$. It remains to prove the convergence of the first term. Take $C(t)$ such that $\int_{D(0, t)}|\log | z-$ $\zeta / \zeta \| d m(\zeta) \leq C(t)$ and choose $p$ big enough so that $\left|\mu_{p}(\zeta)-\mu^{*}(\zeta)\right| \leq \varepsilon / C(t)$ uniformly on $D(0, t)$. Then the estimate follows.

We know that the sequence of distance functions $d_{\phi_{x_{n}}}$ has a subsequence converging to $d_{\phi^{*}}$ uniformly on compact sets of $\mathbb{C} \times \mathbb{C}$, because the $\rho_{x_{n_{k}}}$ converge uniformly. By construction $\rho_{x_{n}}(0)=1$. On the other hand, the Lipschitz property (6) implies that the $\rho_{x_{n_{k}}}$ are equibounded on any compact. Moreover, by Lemma 4 , they are equicontinous on compact sets. We can thus extract again a convergent subsequence.

Corollary 25. Given a subharmonic function $\phi$ with doubling Laplacian, $\Lambda$ a $\rho$-separated subsequence and $\left\{z_{n}\right\}_{n \in \mathbb{N}}$ a sequence of complex numbers, there exist a subharmonic function $\phi^{*}$, a $\rho_{\phi^{*}}$-separated sequence $\Lambda^{*}$ and a subsequence $\left\{x_{n}\right\}_{n \in \mathbb{N}}$ of $\left\{z_{n}\right\}_{n \in \mathbb{N}}$ such that $\Lambda_{x_{n}} \rightarrow \Lambda^{*}$, and $\phi_{x_{n}} \rightarrow \phi^{*}$ and $\Delta \phi_{x_{n}} \rightarrow \Delta \phi^{*}$ uniformly on compact sets.

We will write $\left(\Lambda_{x_{n}}, \phi_{x_{n}}\right) \rightarrow\left(\Lambda^{*}, \phi^{*}\right)$. The set of all such weak limits will be denoted by $W(\Lambda, \phi)$.

Let us prove now the stability of the upper and lower densities with respect to weak limits.

Lemma 26. Let $\Lambda$ be a $\rho$-separated sequence, $\left\{x_{n}\right\}_{n} \subset \mathbb{C}$, and assume that $\left(\Lambda_{x_{n}}, \phi_{x_{n}}\right) \rightarrow$ $\left(\Lambda^{*}, \phi^{*}\right)$. Then

(a) $\mathcal{D}_{\Delta \phi}^{+}(\Lambda)<1 / 2 \pi$ implies $\mathcal{D}_{\Delta \phi^{*}}^{+}\left(\Lambda^{*}\right)<1 / 2 \pi$.

(b) $\mathcal{D}_{\Delta \phi}^{-}(\Lambda)>1 / 2 \pi$ implies $\mathcal{D}_{\Delta \phi^{*}}^{-}\left(\Lambda^{*}\right)>1 / 2 \pi$.

Proof. Denote $\Lambda_{n}=\Lambda_{x_{n}}, \phi_{n}=\phi_{x_{n}}$ and $\rho_{n}=\rho_{x_{n}}$. By hypothesis $\left\{\Delta \phi_{n}\right\}_{n} \rightarrow \Delta \phi^{*}$ uniformly on compact sets, and therefore $\left\{\rho_{n}\right\}_{n} \rightarrow \rho^{*}$ also uniformly on compact sets. Thus, for any $\epsilon(r)>0$,

$$
\begin{aligned}
& \frac{n_{\Lambda^{*}}\left(z,(r-\epsilon(r)) \rho_{\phi^{*}}(z)\right)}{\int_{D_{\phi^{*}}^{r}(z)} \Delta \phi^{*}} \leq \liminf _{n \rightarrow \infty} \frac{n_{\Lambda_{n}}\left(z, r \rho_{n}(z)\right)}{\int_{D_{\phi_{n}}^{r}(z)} \Delta \phi_{n}} \leq \\
\leq & \limsup _{n \rightarrow \infty} \frac{n_{\Lambda_{n}}\left(z, r \rho_{n}(z)\right)}{\int_{D_{\phi_{n}}^{r}(z)} \Delta \phi_{n}} \leq \frac{n_{\Lambda^{*}}\left(z,(r+\epsilon(r)) \rho_{\phi^{*}}(z)\right)}{\int_{D_{\phi^{*}}^{r}(z)} \Delta \phi^{*}} .
\end{aligned}
$$


(a) Since $\mathcal{D}_{\Delta \phi}^{+}(\Lambda)<1 / 2 \pi$, there exist $\varepsilon, R_{0}>0$ such that, if $w=\tau_{x_{n}}^{-1}(z)$

$$
\frac{n_{\Lambda_{n}}\left(w, r \rho_{n}(w)\right)}{\int_{D_{\phi_{n}}^{r}(w)} \Delta \phi_{n}}=\frac{n_{\Lambda}(z, r \rho(z))}{\int_{D_{\phi}^{r}(z)} \Delta \phi} \leq 1 / 2 \pi-\varepsilon \quad \forall r>R_{0}, \forall n \in \mathbb{N}, \forall w \in \mathbb{C} .
$$

Taking limits as $n \rightarrow \infty$ and picking $\epsilon(r)$ so that $\epsilon(r) / r \rightarrow 0$ we see, using Lemma 11, that $\mathcal{D}_{\Delta \phi^{*}}^{+}\left(\Lambda^{*}\right)<1 / 2 \pi$.

(b) is proved similarly.

\section{PRELIMINARY PROPERTIES OF SAMPLING AND INTERPOLATING SEQUENCES}

This section is devoted to prove auxiliary results on interpolating and sampling sequences. A main result is that there do not exist sequences which are simultaneously sampling and interpolating. We also prove some results on inclusions between spaces of sampling and interpolating sequences for various weights.

An easy consequence of Lemma 19 is that we only need to deal with $\rho$-separated sequences.

Lemma 27. Let $\Lambda \subset \mathbb{C}$.

(a) If $\Lambda \in \operatorname{Int} \mathcal{F}_{\phi}^{p}$, then $\Lambda$ is $\rho$-separated.

(b) If $\Lambda \in \operatorname{Samp} \mathcal{F}_{\phi}^{p}$, there exists a $\rho$-separated subsequence $\Lambda^{\prime} \subset \Lambda$ such that $\Lambda^{\prime} \in$ $\operatorname{Samp} \mathcal{F}_{\phi}^{p}$.

(c) If $p<\infty$ and $\Lambda \in \operatorname{Samp} \mathcal{F}_{\phi}^{p}$, then $\Lambda$ is a finite union of $\rho$-separated sequences.

(d) Let $\Lambda \in \operatorname{Samp} \mathcal{F}_{\phi}^{p}$ be $\rho$-separated. There exists $r>0$ such that $\mathbb{C}=\cup_{\lambda \in \Lambda} D^{r}(\lambda)$.

Proof. (a) Assume that $\lambda, \mu \in \Lambda$ with $|\lambda-\mu| \leq \rho(\lambda)$ and take $f \in \mathcal{F}_{\phi}^{p}$ such that $f(\lambda)=e^{\phi(\lambda)}$, $f(\mu)=0$ and $\|f\|_{\mathcal{F}_{\phi}^{p}} \lesssim 1$. Then

$$
1=|| f(\lambda)\left|e^{-\phi(\lambda)}-\right| f(\mu)\left|e^{-\phi(\mu)}\right| \lesssim\left|\nabla\left(|f| e^{-\phi}\right)(\zeta)\right||\mu-\lambda| .
$$

The result follows then from Lemma 19 (b).

(b) As in the proof of [Beu89, Theorem 2, p. 344], using here Lemma 19,b) instead of Bernstein's theorem, we get

$$
\left|\frac{1}{L_{\phi}^{p}(\Lambda)}-\frac{1}{L_{\phi}^{p}\left(\Lambda^{\prime}\right)}\right| \leq C\left[\Lambda, \Lambda^{\prime}\right] .
$$

(c) It is enough to show that there exists $r>0$ and $M$ such that $\#\left(D^{\eta}(z) \cap \Lambda\right) \leq M$ for all $z \in \mathbb{C}$. To this end, consider the function $f_{z}(\zeta)=e^{\phi}(z) P_{z}(\zeta)$, where $P_{z}$ is given by Theorem 18 (with $\varepsilon=1$ ). We have $\left\|f_{z}\right\|_{\mathcal{F}_{\phi}^{p}} \leq C$, and for $r$ small enough $\left|f_{z}(\zeta)\right| \gtrsim e^{\phi}(\zeta)$ in $D^{r}(z)$. So the left sampling inequality (see (2) ) yields

$$
\#\left(D^{r}(z) \cap \Lambda\right) \leq\left\|f_{z} \mid \Lambda\right\|_{\ell_{\phi}^{p}(\Lambda)} \leq C L_{\phi}^{p}(\Lambda) .
$$

(d) It is enough to see that for $s$ big enough $\Lambda \cap D^{s}(z) \neq \emptyset$ for all $z \in \mathbb{C}$. 
Take $f_{z}$ as in (c). Let $\varepsilon>0$ be the $\rho$-separation of $\Lambda$. Since

$$
\left|f_{z}(\zeta)\right|^{p} e^{-p \phi(\zeta)} \rho^{-2}(\zeta) \lesssim \frac{\Delta \phi(\zeta)}{1+d_{\phi}^{m}(z, \zeta)}
$$

Lemma 19 a) and Lemma 9 lead to

$$
\sum_{\lambda \notin D^{s}(z)}\left|f_{z}(\lambda)\right|^{p} e^{-p \phi(\lambda)} \lesssim \sum_{\lambda \notin D^{s}(z)} \int_{D^{\varepsilon}(\lambda)} \frac{\Delta \phi(\zeta)}{1+d_{\phi}^{m}(z, \zeta)} \lesssim \int_{\zeta \notin D^{s-\epsilon(s)}(z)} \frac{\Delta \phi(\zeta)}{1+d_{\phi}^{m}(z, \zeta)}
$$

According to Remark 2 this tends to 0 uniformly in $z$ as $s$ goes to $\infty$. Thus, for $s$ big enough the sampling inequality gives

$$
1 \leq C \sum_{\lambda \in \Lambda \cap D^{s}(z)}\left|f_{z}(\lambda)\right|^{p} e^{-p \phi(\lambda)}
$$

In particular $\Lambda \cap D^{s}(z) \neq \emptyset$, as desired.

4.1. Weak limits and interpolating and sampling sequences. In this section $\tau_{x}^{\phi}$ will denote the scaled translation associated to the weight $\phi$, as described in Section 3.4. The main result is as follows.

Proposition 28. Let $\phi$ a subharmonic function with doubling Laplacian and $\Lambda$ be a $\rho$-separated sequence. Assume $\left(\Lambda^{*}, \phi^{*}\right) \in W(\Lambda, \phi)$.

(a) If $\Lambda \in \operatorname{Samp} \mathcal{F}_{\phi}^{p}$ then $\Lambda^{*} \in \operatorname{Samp} \mathcal{F}_{\phi^{*}}^{p}$.

(b) If $\Lambda \in \operatorname{Int} \mathcal{F}_{\phi}^{p}$ then $\Lambda^{*} \in \operatorname{Int} \mathcal{F}_{\phi^{*}}^{p}$.

Proof. (a) We argue by contradiction. Otherwise there exist $\varepsilon_{n}>0$ decreasing to zero and functions $f_{n} \in \mathcal{F}_{\phi^{*}}^{p}$ such that $\left\|f_{n}\right\|_{\mathcal{F}_{\phi^{*}}^{p}}=1$ and $\left\|f_{n} \mid \Lambda^{*}\right\|_{\ell_{\phi^{*}}^{p}\left(\Lambda^{*}\right)} \leq \varepsilon_{n}$.

By Corollary 25 there exists a sequence $\left\{x_{j}\right\}_{j \in \mathbb{N}}$ in $\mathbb{C}$ such that $\left(\Lambda_{j}, \phi_{j}\right) \rightarrow\left(\Lambda^{*}, \phi^{*}\right)$, where we denote $\Lambda_{j}:=\Lambda_{x_{j}}$ and $\phi_{j}:=\phi_{x_{j}}$.

For every $n$ consider $s_{n}$ big enough so that if $D_{n}:=D_{\phi^{*}}^{s_{n}}(0)$ then $\left\|f_{n} \mid D_{n}\right\|_{\mathcal{F}_{\phi^{*}}^{p}} \geq 1-\varepsilon_{n}$. Set $\widetilde{D}_{n}:=D_{\phi^{*}}^{s_{n}^{2}}(0)$.

We claim that there exists a smooth cut-off function $\mathcal{X}_{n}$ such that $\mathcal{X}_{n}(\zeta)=1$ in $D_{n}, \mathcal{X}_{n}(\zeta)=0$ in $\mathbb{C} \backslash \widetilde{D}_{n}$ and $\left|\bar{\partial} \mathcal{X}_{n}\right| \leq \varepsilon_{n} / \rho_{\phi^{*}}$. To see this start with a smooth $\mathcal{X}_{n}$ depending linearly on $|\zeta|$ on $s_{n} \leq|\zeta| \leq s_{n}^{2}$. Then

$$
\left|\bar{\partial} \mathcal{X}_{n}(\zeta)\right| \leq \frac{1}{\rho_{\phi^{*}}(0)\left(s_{n}^{2}-s_{n}\right)}
$$

By Lemma $2 \rho_{\phi^{*}}(\zeta) / \rho_{\phi^{*}}(0) \leq s_{n}^{2(1-\delta)}$ for some $\delta \in(0,1)$. Thus, if $s_{n}$ is big enough

$$
\left|\bar{\partial} \mathcal{X}_{n}(\zeta)\right| \leq \frac{s_{n}^{2(1-\delta)}}{\rho_{\phi^{*}}(\zeta)\left(s_{n}^{2}-s_{n}\right)} \leq \frac{\varepsilon_{n}}{\rho_{\phi^{*}}(\zeta)}
$$


Take now $j_{n}$ big enough so that $\rho_{\phi_{j_{n}}} / \rho_{\phi^{*}} \leq 2$ on $\widetilde{D}_{n}$ and

$$
\begin{aligned}
&\left|\left\|f_{n}\left|\widetilde{D}_{n}\left\|_{\mathcal{F}_{\phi_{n}}^{p}}-\right\| f_{n}\right| \widetilde{D}_{n}\right\|_{\mathcal{F}_{\phi^{*}}^{p}}\right| \leq \varepsilon_{n}, \\
&\left|\left\|f_{n}\left|\Lambda_{j_{n}} \cap \widetilde{D}_{n}\left\|_{\ell_{\phi_{j_{n}}}^{p}}-\right\| f_{n}\right| \Lambda^{*} \cap \widetilde{D}_{n}\right\|_{\ell_{\phi^{*}}^{p}}\right| \leq \varepsilon_{n} .
\end{aligned}
$$

Define $g_{n}=f_{n} \mathcal{X}_{n}$. Then $\bar{\partial} g_{n}$ is supported on $C_{n}:=\left\{s_{n} \leq|\zeta| \leq s_{n}^{2}\right\}$ and $\left|\bar{\partial} g_{n}(\zeta)\right| \leq$ $\varepsilon_{n}\left|f_{n}(\zeta)\right| / \rho_{\phi^{*}}(\zeta)$, so by Theorem 1 there exists $u_{n}$ solution to $\bar{\partial} u_{n}=\bar{\partial} g_{n}$ with

$$
\left\|u_{n}\right\|_{\mathcal{F}_{\phi_{j_{n}}}^{p}} \lesssim\left\|\bar{\partial} g_{n} \rho_{\phi_{j_{n}}}\right\|_{\mathcal{F}_{\phi_{j_{n}}}^{p}} \lesssim \varepsilon_{n}\left\|f_{n} \mid \widetilde{D}_{n}\right\|_{\mathcal{F}_{\phi_{j_{n}}}^{p}} \lesssim \varepsilon_{n}
$$

The function $G_{n}=g_{n}-u_{n}$ is holomorphic and satisfies

$$
\left\|G_{n}\right\|_{\mathcal{F}_{\phi_{j_{n}}}^{p}} \geq\left\|f_{n} \mid D_{n}\right\|_{\mathcal{F}_{\phi_{j_{n}}}^{p}}-\left\|u_{n}\right\|_{\mathcal{F}_{\phi_{j_{n}}}^{p}} \geq 1-C \varepsilon_{n} \simeq 1
$$

We will check now that $G_{n} \mid \Lambda_{j_{n}}$ is small. Split $\Lambda_{j_{n}}$ into $\left.\widetilde{\Lambda}_{j_{n}}=\Lambda_{j_{n}} \cap\left\{D_{n} \cup\left(\mathbb{C} \backslash \widetilde{D}_{n}\right)\right)\right\}$ and $\widehat{\Lambda}_{j_{n}}=\Lambda_{j_{n}} \backslash \widetilde{\Lambda}_{j_{n}}$. On the one hand

$$
\left\|G_{n}\left|\widetilde{\Lambda}_{j_{n}}\left\|_{\ell_{\phi_{j_{n}}}^{p}\left(\widetilde{\Lambda}_{j_{n}}\right)} \leq\right\| f_{n}\right| \widetilde{D}_{n} \cap \widetilde{\Lambda}_{j_{n}}\right\|_{\ell_{\phi_{j_{n}}}^{p}\left(\widetilde{\Lambda}_{j_{n}}\right)}+\left\|u_{n} \mid \widetilde{\Lambda}_{j_{n}}\right\|_{\ell_{\phi_{j_{n}}}^{p}\left(\widetilde{\Lambda}_{j_{n}}\right)} .
$$

From $\left\|u_{n} \mid \widetilde{\Lambda}_{j_{n}}\right\|_{\ell_{\phi_{j_{n}}}^{p}\left(\widetilde{\Lambda}_{j_{n}}\right)} \leq\left\|u_{n}\right\|_{\mathcal{F}_{\phi_{j_{n}}}^{p}} \leq \varepsilon_{n}$ (by Lemma 19 for the case $p<\infty$, since $u$ is holomorphic in $\left.D_{n} \cup\left(\mathbb{C} \backslash \widetilde{D}_{n}\right)\right)$ we deduce that $\left\|G_{n} \mid \widetilde{\Lambda}_{j_{n}}\right\|_{\ell_{\phi_{j_{n}}^{p}}\left(\widetilde{\Lambda}_{j_{n}}\right)} \lesssim \varepsilon_{n}$. On the other hand

$$
\left\|G_{n}\left|\widehat{\Lambda}_{j_{n}}\left\|_{\ell_{\phi_{j_{n}}}^{p}\left(\widehat{\Lambda}_{j_{n}}\right)} \lesssim\right\| G_{n}\right|\left(\widetilde{D}_{n} \backslash D_{n}\right)\right\|_{\mathcal{F}_{\phi_{j_{n}}}^{p}} \lesssim\left\|\left|f_{n}\right|+\left|u_{n}\right| \mid\left(\widetilde{D}_{n} \backslash D_{n}\right)\right\|_{\mathcal{F}_{\phi_{j_{n}}}^{p}} \lesssim \varepsilon_{n} .
$$

This together with the above and the fact that the sampling constants of $\Lambda$ and $\Lambda_{j_{n}}$ coincide (Lemma 23,b)) leads to contradiction.

(b) Assume that $\Lambda^{*}=\left\{\lambda_{k}^{*}\right\}_{k}$, and let $v \in \ell_{\phi}^{p}\left(\Lambda^{*}\right)$ with $\|v\|_{\ell_{\phi}^{p}\left(\Lambda^{*}\right)} \leq 1$. Let also $\Lambda_{j}=\left\{\lambda_{k}^{j}\right\}_{k}$ be such that $\Lambda_{j} \rightarrow \Lambda^{*}$ uniformly on compact sets. For $\varepsilon_{n}$ decreasing to zero and $s_{n}$ big enough (to be chosen later) there exists $j_{n}$ such that $\|v\|_{\ell_{\phi_{j_{n}}}^{p}\left(\Lambda_{j_{n}} \cap D_{\phi^{*}}^{s_{n}}(0)\right)} \leq 2$ and

$$
\frac{e^{-\phi^{*}} \rho_{\phi^{*}}^{-2 / p}}{e^{-\phi_{j_{n}}} \rho_{\phi_{j_{n}}}^{-2 / p}} \leq 2 \quad \text { on } \quad D_{\phi^{*}}^{s_{n}^{2}}(0) \text {. }
$$

Since the interpolation constant $M\left(\Lambda_{j}\right)$ does not depend on $j$ there exist $f_{n} \in \mathcal{F}_{\phi_{j_{n}}}^{p}$ with $\left\|f_{n}\right\|_{\mathcal{F}_{\phi_{j_{n}}}^{p}} \leq 2 M(\Lambda)$ and

$$
f_{n}\left(\lambda_{k}^{j_{n}}\right)= \begin{cases}v_{k} & \text { if } \lambda_{k}^{j_{n}} \in D_{\phi^{*}}^{s_{n}}(0) \\ 0 & \text { otherwise. }\end{cases}
$$

We will now use the same technique as in (a) to modify $f_{n}$ so that it falls in $\mathcal{F}_{\phi^{*}}^{p}$. Take the cut-off function $\mathcal{X}_{n}$ constructed above, define $g_{n}=f_{n} \mathcal{X}_{n}$ and consider a solution $u_{n}$ to $\bar{\partial} u_{n}=f_{n} \bar{\partial}\left(\mathcal{X}_{n}\right)$ 
such that:

$$
\begin{aligned}
\left\|u_{n}\right\|_{\mathcal{F}_{\phi^{*}}^{p}} & \lesssim\left\|f_{n} \bar{\partial}\left(\mathcal{X}_{n}\right) \rho_{\phi^{*}}\right\|_{\mathcal{F}_{\phi^{*}}^{p}} \lesssim \varepsilon_{n}\left\|f_{n} \mid D_{\phi^{*}}^{s_{n}^{2}}(0)\right\|_{\mathcal{F}_{\phi^{*}}^{p}} \lesssim \varepsilon_{n}\left\|f_{n}\right\|_{\mathcal{F}_{\phi_{j_{n}}}^{p}} \lesssim \varepsilon_{n}, \\
\left\|u_{n}\right\|_{\mathcal{F}_{\phi^{*}}^{\infty}} & \lesssim\left\|f_{n} \bar{\partial}\left(\mathcal{X}_{n}\right) \rho_{\phi^{*}}\right\|_{\mathcal{F}_{\phi^{*}}^{\infty}} \lesssim \varepsilon_{n}\left\|f_{n}\right\|_{\mathcal{F}_{\phi^{*}}^{p}} \lesssim \varepsilon_{n} .
\end{aligned}
$$

According to Theorem $\mathrm{C}$ and (14) such a solution always exists.

The entire function $G_{n}=f_{n} \bar{\partial}\left(\mathcal{X}_{n}\right)-u_{n}$ is $\mathcal{F}_{\phi^{*}}^{p}$ and $\left\|G_{n}\right\|_{\mathcal{F}_{\phi^{*}}^{p}} \leq C M$. By Montel's theorem we may assume that $G_{n}$ converges to a function $G \in \mathcal{F}_{\phi^{*}}^{p}$. Notice that $G_{n}\left(\lambda_{k}^{j_{n}}\right)=v_{k}-u_{n}\left(\lambda_{k}^{j_{n}}\right)$ for $\lambda_{k}^{j_{n}} \in D_{\phi^{*}}^{s_{n}}(0)$, and by the $L^{\infty}$ estimates, $\left|u_{n}\left(\lambda_{k}^{j_{n}}\right)\right|$ tends to zero as $n$ goes to infinity. Therefore $G$ interpolates $v$.

The next lemma and its corollary are our counterparts to equivalent results of Beurling in the context of the Bernstein space, see [Beu89, p. 345].

Lemma 29. Suppose that for every weak limit $\left(\Lambda^{*}, \phi^{*}\right) \in W(\Lambda, \phi)$ the sequence $\Lambda^{*}$ is a uniqueness set for $\mathcal{F}_{\phi^{*}}^{\infty}$. Then there exists $\varepsilon>0$ such that $\Lambda$ is sampling for $\mathcal{F}_{(1+\varepsilon) \phi^{*}}^{\infty}$

Proof. If this is not the case there exist $\varepsilon_{n}>0$ decreasing to $0, f_{n} \in \mathcal{F}_{\left(1+\varepsilon_{n}\right) \phi}^{\infty}$ and $z_{n} \in \mathbb{C}$ such that $\left|f_{n}\left(z_{n}\right)\right| e^{-\left(1+\varepsilon_{n}\right) \phi\left(z_{n}\right)}=1,\left\|f_{n}\right\|_{\mathcal{F}_{\left(1+\varepsilon_{n}\right) \phi}^{\infty}} \leq 2$ and $\left\|f_{n} \mid \Lambda\right\|_{\ell_{\left(1+\varepsilon_{n}\right) \phi}^{\infty}(\Lambda)} \leq \varepsilon_{n}$.

Denote $\psi_{n}=\left(1+\varepsilon_{n}\right) \phi$. Let $\Lambda_{n}=\left(\tau_{z_{n}}^{\psi_{n}}\right)^{-1}(\Lambda)$ and $g_{n}=T_{z_{n}}^{\psi_{n}} f_{n}$. Then, denoting $\psi_{n, z_{n}}=(1+$ $\left.\varepsilon_{n}\right) \phi_{z_{n}}$, we have $\left|g_{n}(0)\right|=1$ and $\left\|g_{n}\left|\Lambda_{n}\left\|_{\ell_{\psi_{n}, z_{n}}^{\infty}\left(\Lambda_{n}\right)}=\right\| f_{n}\right| \Lambda\right\|_{\ell_{\psi_{n}}^{\infty}(\Lambda)} \leq \varepsilon_{n}$. Taking a subsequence if necessary, we can assume that $\Lambda_{n}$ converges weakly to $\Lambda^{*}, \psi_{n, z_{n}} \rightarrow \phi^{*}$ uniformly on compact sets and $g_{n} \rightarrow g^{*} \in \mathcal{F}_{\phi^{*}}^{\infty}$ (by Montel's Theorem). So $g^{*}$ vanishes on $\Lambda^{*}$ and $\left|g^{*}(0)\right|=1$, contradicting the fact that $\Lambda^{*}$ is a uniqueness sequence.

Corollary 30. Let $\phi$ a subharmonic function with doubling Laplacian and let $\Lambda$ be a $\rho$-separated sequence. The sequence $\Lambda$ is in Samp $\mathcal{F}_{\phi}^{\infty}$ if and only if for all weak limit $\left(\Lambda^{*}, \phi^{*}\right) \in W(\Lambda, \phi)$, the sequence $\Lambda^{*}$ is a uniqueness set for $\mathcal{F}_{\phi^{*}}^{\infty}$.

4.2. Non-existence of simultaneously sampling and interpolating sequences. An important result in the proof of Theorems A and B is the following theorem. It is an analogous result to [Sei92, Lemma 6.2].

Theorem 31. There is no sequence $\Lambda$ both sampling and interpolating for $\mathcal{F}_{\phi}^{p}, p \in[1, \infty]$.

Proof. Assume that such sequence $\Lambda$ exists. We claim that

$$
\sup _{\lambda^{*} \in \Lambda} \sum_{\lambda \in \Lambda \backslash \lambda^{*}} \frac{\rho(\lambda) \rho\left(\lambda^{*}\right)}{\left|\lambda-\lambda^{*}\right|^{2}}<\infty .
$$

Let $p \in[1, \infty)$. Given any $\lambda^{*} \in \Lambda$ take a function $g$ such that $g\left(\lambda^{*}\right)=1, g(\lambda)=0$ for $\lambda \neq \lambda^{*}$ and $\|g\|_{\mathcal{F}_{\phi}^{p}} \lesssim e^{-p \phi\left(\lambda^{*}\right)}$. Such $g$ exists because $\Lambda$ is interpolating. Consider the function

$$
F(z)=\sum_{\lambda \in \Lambda \backslash \lambda^{*}} \rho(\lambda) \frac{g(z)\left(z-\lambda^{*}\right)}{(z-\lambda) \overline{\left(\lambda^{*}-\lambda\right)}} .
$$


The sampling inequality shows that $F \in \mathcal{F}_{\phi}^{p}$. Moreover, since $|F(\lambda)|=\left|g^{\prime}(\lambda)\right| \rho(\lambda)$ for all $\lambda \in \Lambda \backslash \lambda^{*}$ and $F\left(\lambda^{*}\right)=0$, we have

$$
\|F\|_{\mathcal{F}_{\phi}^{p}}^{p} \lesssim \sum_{\lambda \in \Lambda \backslash \lambda^{*}}\left|g^{\prime}(\lambda)\right|^{p} \rho^{p}(\lambda) e^{-p \phi(\lambda)}
$$

We use now Lemma 20 and the fact that $\Lambda$ is $\rho$-separated (since it is interpolating):

$$
\|F\|_{\mathcal{F}_{\phi}^{p}}^{p} \lesssim \sum_{\Lambda \backslash \lambda^{*}} \int_{D(\lambda)}|g|^{p} e^{-p \phi} d \sigma \lesssim\|g\|_{\mathcal{F}_{\phi}^{p}}^{p} \lesssim e^{-p \phi\left(\lambda^{*}\right)}
$$

We want to estimate $\left|F^{\prime}\left(\lambda^{*}\right)\right|$. Using again Lemma 20

$$
\left|F^{\prime}\left(\lambda^{*}\right)\right|^{p} e^{-p \phi\left(\lambda^{*}\right)} \rho^{p}\left(\lambda^{*}\right) \lesssim \int_{D(\lambda)}|F|^{p} e^{-p \phi} d \sigma \lesssim e^{-p \phi\left(\lambda^{*}\right)}\left(\lambda^{*}\right)
$$

Therefore $\left|F^{\prime}\left(\lambda^{*}\right)\right| \rho\left(\lambda^{*}\right) \lesssim 1$. On the other hand

$$
F^{\prime}\left(\lambda^{*}\right)=\sum_{\lambda \in \Lambda \backslash \lambda^{*}} \frac{\rho(\lambda)}{\left|\lambda-\lambda^{*}\right|^{2}}
$$

This yields (15). The obvious modifications give (15) in the case $p=\infty$.

According to Lemma 27(d) there exists $r>0$ with $\mathbb{C}=\cup_{\lambda \in \Lambda} D^{r}(\lambda)$. Also, there exists $r_{0}>0$ depending on $r$ such that,

$$
\int_{D^{r}(\lambda) \backslash D^{r_{0}\left(\lambda^{*}\right)}} \frac{d m(z)}{1+\left|z-\lambda^{*}\right|^{2}} \leq C(r) \frac{\rho^{2}(\lambda)}{\left|\lambda-\lambda^{*}\right|^{2}} \quad \forall \lambda \notin D^{r_{0}}\left(\lambda^{*}\right) .
$$

We may now finish by taking a big disk $D(0, M)$ and $\lambda_{M}^{*} \in D(0, M)$ in such a way that $\rho\left(\lambda_{M}^{*}\right) \geq$ $\rho(\lambda)$ for all $\lambda \in \Lambda \cap D(0, M)$. In this case

$$
\int_{D(0, M) \backslash D^{r_{0}\left(\lambda_{M}^{*}\right)}} \frac{d m(z)}{1+\left|z-\lambda_{M}^{*}\right|^{2}} \lesssim \sum_{\substack{\lambda \in \Lambda \\ \lambda \notin D^{r_{0}}\left(\lambda^{*}\right)}} \int_{D^{r}(\lambda)} \frac{d m(z)}{1+\left|z-\lambda_{M}^{*}\right|^{2}} \lesssim \sum_{\lambda \in \Lambda \backslash \lambda_{M}^{*}} \frac{\rho(\lambda) \rho\left(\lambda_{M}^{*}\right)}{\left|\lambda-\lambda_{M}^{*}\right|^{2}}<C .
$$

This is a contradiction, since $\lim _{M \rightarrow \infty} \rho\left(\lambda_{M}^{*}\right) / M=0$ and the left hand side of the previous inequality tends to $\infty$ as $M$ goes to $\infty$.

Corollary 32. Any sequence obtained by deleting a finite number of points of $\Lambda \in \operatorname{Samp} \mathcal{F}_{\phi}^{p}$ is still in $\operatorname{Samp} \mathcal{F}_{\phi}^{p}$.

Proof. Suppose that we remove a point $\lambda$ from a sampling sequence $\Lambda$ and the resulting sequence $\Lambda^{\prime}=\Lambda \backslash \lambda$ is no longer sampling. Then there is a sequence $f_{n} \in \mathcal{F}_{\phi}^{p}$ such that $\left\|f_{n}\right\|_{\mathcal{F}_{\phi}^{p}}=1$ and $\left\|\left.f\right|_{\Lambda^{\prime}}\right\|_{\ell_{\phi}^{p}} \leq 1 / n$. Since $\Lambda$ is sampling $\left|f_{n}(\lambda)\right|$ is bounded from bellow and above. Therefore we can take a subsequence converging to $f \in \mathcal{F}_{\phi}^{p}$ such that $\left.f\right|_{\Lambda^{\prime}}=0$ and $f(\lambda) \neq 0$. Now for any $\mu \in \Lambda$ we consider the functions $g_{\mu}(z)=f(z)(z-\lambda) /(z-\mu)^{k}$ where $k$ is the multiplicity of 
$f$ at $\mu$. This functions belong to $\mathcal{F}_{\phi}^{p}$ and $g_{\mu}(\lambda)=0$ if $\lambda \neq \mu$, thus for any values $v \in \ell_{\phi}^{p}$ we can construct the function

$$
h(z)=\sum_{\Lambda} v_{\lambda} g_{\lambda}(z) / g_{\lambda}(\lambda) .
$$

Since $\Lambda$ is sampling $h \in \mathcal{F}_{\phi}^{p}$ and it interpolates the values $v$. Therefore $\Lambda \in \operatorname{Int} \mathcal{F}_{\phi}^{p}$, a contradiction with Theorem 31 .

We want to prove next an analogue for interpolating sequences: adding a finite number of points to an interpolating sequence gives again an interpolating sequence.

Given $\Lambda$ and a point $z$ define, following [Beu89, pp. 352-354]

$$
\sigma_{\phi}^{p}(z, \Lambda):=\sup \left\{|f(z)| e^{-\phi(z)},\|f\|_{\mathcal{F}_{\phi}^{p}} \leq 1, f \mid \Lambda \equiv 0\right\} .
$$

Notice first that if $\Lambda$ is interpolating and $z \notin \Lambda$ this is strictly positive. Indeed, $\Lambda$ is not a uniqueness sequence, otherwise $\Lambda$ would be also sampling, contradicting Theorem 31 . Thus there exists $f \in \mathcal{F}_{\phi}^{p}, f \neq 0$ with $f \mid \Lambda \equiv 0$ and, eventually dividing $f$ by a power of $(\zeta-z)$, $f(z) \neq 0$. Hence $\sigma_{\phi}^{p}(z, \Lambda)>0$.

Lemma 33. Let $\Lambda \in \operatorname{Int} \mathcal{F}_{\phi}^{p}$. Then $\Lambda \cup\{z\} \in \operatorname{Int} \mathcal{F}_{\phi}^{p}$ for all $z \notin \Lambda$. Furthermore, for all $\varepsilon>0$ there exists $C>0$ such that $d_{\phi}(\Lambda, z) \geq \varepsilon$ implies $M_{\phi}^{p}(\Lambda \cup\{z\}) \leq C M_{\phi}^{p}(\Lambda)$.

Proof. As in the proof of [Beu89, Lemma 4, p. 353], we have

$$
M_{\phi}^{p}(\Lambda \cup\{z\}) \leq \frac{1+2 M_{\phi}^{p}(\Lambda)}{\sigma_{\phi}^{p}(z, \Lambda)} .
$$

Thus we will be done if we prove that there exists $A>0$ such that $d_{\phi}(z, \Lambda) \geq \varepsilon$ implies $\sigma_{\phi}^{p}(z, \Lambda) \geq A$.

If this is not true, there exists a sequence $\left\{z_{n}\right\} \in \mathbb{C}$ with $d_{\phi}\left(z_{n}, \Lambda\right) \geq \varepsilon$ and $\sigma_{\phi}^{p}\left(z_{n}, \Lambda\right) \leq 1 / n$. Transferring $z_{n}$ to the origin by $\tau_{z_{n}}^{-1}$ (see Section 22), we get a sequence $\Lambda_{n}:=\Lambda_{z_{n}}$ such that $|\lambda| \geq \varepsilon$ for all $\lambda \in \Lambda_{n}$ and $\sigma_{\phi_{n}}^{p}\left(0, \Lambda_{n}\right) \leq 1 / n$, where $\phi_{n}=\phi_{z_{n}}$.

Taking a subsequence if necessary, assume that $\left(\Lambda_{n}, \phi_{n}\right)$ converges to $\left(\Lambda^{*}, \phi^{*}\right)$. By Proposition 28. $\Lambda^{*} \cup\{0\} \in \operatorname{Int} \mathcal{F}_{\phi^{*}}^{p}$, so there exists $f \in \mathcal{F}_{\phi^{*}}^{p}$ with $f \mid \Lambda^{*}=0$ and $|f(0)|=1$. Arguing as in the proof of Proposition 28 we see that there exist $f_{n} \in \mathcal{F}_{\phi_{n}}^{p}$ and $\varepsilon_{n}$ decreasing to zero such that

$$
\left\|f_{n}\left|\Lambda_{n} \|_{\ell_{\phi_{n}}^{p}\left(\Lambda_{n}\right)} \leq \varepsilon_{n}, \quad\right| f_{n}(0) \mid \geq c \quad \text { and } \quad\right\| f_{n} \|_{\mathcal{F}_{\phi_{n}}^{p}} \leq C .
$$

Since $\Lambda_{n}$ is interpolating, there exist also $g_{n} \in \mathcal{F}_{\phi_{n}}^{p}$ with

$$
g_{n}\left|\Lambda_{n}=f_{n}\right| \Lambda_{n} \quad \text { and } \quad\left\|g_{n}\right\|_{\mathcal{F}_{\phi_{n}}^{p}} \leq M_{\phi_{n}}^{p}\left(\Lambda_{n}\right)\left\|f_{n} \mid \Lambda_{n}\right\|_{\ell_{\phi_{n}}^{p}\left(\Lambda_{n}\right)} \leq \varepsilon_{n} M(\Lambda) .
$$

Then $h_{n}:=f_{n}-g_{n} \in \mathcal{F}_{\phi_{n}}^{p}$ vanishes on $\Lambda_{n}$ and $\left\|h_{n}\right\|_{\mathcal{F}_{\phi_{n}}^{p}} \leq 2 C$, therefore $\left|h_{n}(0)\right| \lesssim 1 / n$. On the other hand $\left|g_{n}(0)\right| \lesssim \varepsilon_{n}$ and therefore $\left|h_{n}(0)\right| \geq c / 2$, thus contradicting the previous estimate. 
4.3. Inclusions between various spaces of interpolating sequences. We want to study next the relationship between the spaces of interpolating sequences for various weights. We will use the techniques already exploited in [MT00].

We start with the construction of a sort of peak-functions associated to an interpolating sequence. Let $\delta_{\lambda}^{\lambda^{\prime}}$ denote the Kroenecker indicator, i.e. $\delta_{\lambda}^{\lambda^{\prime}}=1$ if $\lambda=\lambda^{\prime}$ and $\delta_{\lambda}^{\lambda^{\prime}}=0$ otherwise.

Lemma 34. Let $\Lambda \in \operatorname{Int} \mathcal{F}_{\phi}^{p}, 1 \leq p \leq \infty$. Given $\varepsilon>0$ and $m \in \mathbb{N}$, there exist $C>0$ and functions $g_{\lambda} \in \mathcal{F}_{(1+\varepsilon) \phi}^{p}$ such that

(a) $g_{\lambda}\left(\lambda^{\prime}\right)=\delta_{\lambda}^{\lambda^{\prime}}$ for all $\lambda, \lambda^{\prime} \in \Lambda$.

(b) $\left\|g_{\lambda}\right\|_{\mathcal{F}_{(1+\varepsilon) \phi}^{p}} \simeq e^{-(1+\varepsilon) \phi(\lambda)}$.

(c) $\left|g_{\lambda}(z)\right| \lesssim e^{(1+\varepsilon)(\phi(z)-\phi(\lambda))} \frac{1}{1+d_{\phi}^{m}(z, \lambda)}$.

(d) For all $v \in \ell_{(1+\varepsilon) \phi}^{p}(\Lambda),\|v\|_{\ell_{(1+\varepsilon) \phi}^{p}(\Lambda)} \lesssim\left\|\sum_{\lambda \in \Lambda} v_{\lambda} g_{\lambda}\right\|_{\mathcal{F}_{(1+\varepsilon) \phi}^{p}} \lesssim\|v\|_{\ell_{(1+\varepsilon) \phi}^{p}(\Lambda)}$.

(e) $\lim _{r \rightarrow \infty} \sup _{\lambda \in \Lambda} e^{p(1+\varepsilon) \phi(\lambda)} \int_{\mathbb{C} \backslash D^{r}(\lambda)}\left|g_{\lambda}(z)\right|^{p} e^{-p(1+\varepsilon) \phi(z)} d \sigma(z)=0$.

Proof. By hypothesis, there exist functions $f_{\lambda} \in \mathcal{F}_{\phi}^{p}$ such that $f_{\lambda}(\mu)=\delta_{\lambda}^{\mu}$ for all $\lambda, \mu \in \Lambda$ and $\left\|f_{\lambda}\right\|_{\mathcal{F}_{\phi}^{p}} \leq M(\Lambda) e^{-\phi(\lambda)}$. Consider the peak functions $P_{\lambda}$ given by Theorem 18 for $\varepsilon>0$, and define $g_{\lambda}=f_{\lambda} P_{\lambda}$. By construction we have (a) and (c).

(b) When $p=\infty$ we have $e^{-(1+\varepsilon) \phi(\lambda)}=e^{-(1+\varepsilon) \phi(\lambda)}\left|g_{\lambda}(\lambda)\right| \leq\left\|g_{\lambda}\right\|_{\mathcal{F}_{(1+\varepsilon) \phi}^{\infty}}$. The remaining inequality is immediate from (c).

Let $p<\infty$. On the one hand, Lemma 19 (a) gives

$$
e^{-(1+\varepsilon) \phi(\lambda)}=e^{-(1+\varepsilon) \phi(\lambda)}\left|g_{\lambda}(\lambda)\right| \lesssim\left(\int_{D(\lambda)}\left|g_{\lambda}\right|^{p} e^{-p(1+\varepsilon) \phi} d \sigma\right)^{1 / p} \lesssim\left\|g_{\lambda}\right\|_{\mathcal{F}_{(1+\varepsilon) \phi}^{p}} .
$$

On the other hand, (c) and Lemma 5 (b) show that for $m$ big enough

$$
\int_{\mathbb{C}}\left|g_{\lambda}\right|^{p} e^{-p(1+\varepsilon) \phi} d \sigma \lesssim e^{-p(1+\varepsilon) \phi(\lambda)}\left[\int_{D(\lambda)} d \sigma(z)+\int_{\mathbb{C} \backslash D(\lambda)} \frac{\Delta \phi(z)}{d_{\phi}^{p m}(z, \lambda)}\right] \lesssim e^{-p(1+\varepsilon) \phi(\lambda)}
$$

(d) Denote $f=\sum_{\lambda} v_{\lambda} g_{\lambda}$. The left inequalities are proved similarly to (b), for

$$
e^{-(1+\varepsilon) \phi(\lambda)}\left|v_{\lambda}\right|=e^{-(1+\varepsilon) \phi(\lambda)}|f(\lambda)| .
$$

For $p=\infty$ and $v \in \ell_{(1+\varepsilon) \phi}^{\infty,}(\Lambda)$ Lemma 6 and (c) yield

$$
e^{-(1+\varepsilon) \phi(z)}\left(\sum_{\lambda \in \Lambda}\left|v_{\lambda} \| g_{\lambda}(z)\right|\right) \lesssim\|v\|_{\ell_{(1+\varepsilon) \phi}^{\infty}(\Lambda)} \sum_{\lambda \in \Lambda} \frac{1}{1+d_{\phi}^{m}(\lambda, z)} \lesssim\|v\|_{\ell_{(1+\varepsilon) \phi}^{\infty}(\Lambda)} .
$$


Let now $p<\infty$. Using the estimate (c) and Jensen's inequality for convex functions (which is legitimate thanks to Lemma 6) we have

$$
\begin{aligned}
|f(z)|^{p} e^{-p(1+\varepsilon) \phi(z)} \rho^{-2}(z) & \lesssim \frac{1}{\rho^{2}(z)}\left[\sum_{\lambda \in \Lambda}\left|v_{\lambda}\right| e^{-(1+\varepsilon) \phi(\lambda)} \frac{1}{1+d_{\phi}^{m}(z, \lambda)}\right]^{p} \\
& \lesssim \frac{1}{\rho^{2}(z)} \sum_{\lambda \in \Lambda}\left|v_{\lambda}\right|^{p} e^{-p(1+\varepsilon) \phi(\lambda)} \frac{1}{1+d_{\phi}^{m}(z, \lambda)}
\end{aligned}
$$

Now we apply Lemma 5 (b) and obtain

$$
\int_{\mathbb{C}}|f|^{p} e^{-p(1+\varepsilon) \phi} d \sigma \lesssim \sum_{\lambda \in \Lambda}\left|v_{\lambda}\right|^{p} e^{-p(1+\varepsilon) \phi(\lambda)} \int_{\mathbb{C}} \frac{\Delta \phi(z)}{1+d_{\phi}^{m}(z, \lambda)} \lesssim\|v\|_{\ell_{(1+\varepsilon) \phi}^{p}}^{p}(\Lambda) .
$$

(e) This follows from (c) and Remark 2, since

$$
e^{p(1+\varepsilon) \phi(\lambda)} \int_{\mathbb{C} \backslash D^{r}(\lambda)}\left|g_{\lambda}(z)\right|^{p} e^{-p(1+\varepsilon) \phi(z)} d \sigma(z) \lesssim \int_{\mathbb{C} \backslash D^{r}(\lambda)} \frac{\Delta \phi(z)}{d_{\phi}^{m}(z, \lambda)} .
$$

Theorem 35. For all $\varepsilon>0$ and $1 \leq p, p^{\prime} \leq \infty$, the following inclusions hold

$$
\operatorname{Int} \mathcal{F}_{\phi}^{p} \subset \operatorname{Int} \mathcal{F}_{(1+\varepsilon) \phi}^{p^{\prime}}
$$

Proof. It will be enough to prove that for all $\varepsilon>0$ and $1 \leq p \leq \infty$,

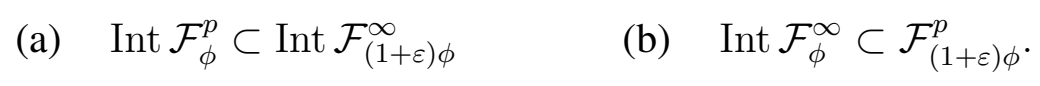

(a) Take the functions $g_{\lambda}$ given by Lemma 34. For $v \in \ell_{(1+\varepsilon) \phi}^{\infty}(\Lambda)$ we consider the interpolating function

$$
f(z)=\sum_{\lambda \in \Lambda} v_{\lambda} g_{\lambda}(z)
$$

A direct estimate using Lemma 34(c) yields

$$
|f(z)| e^{-(1+\varepsilon) \phi(z)} \lesssim \sum_{\lambda \in \Lambda} \frac{1}{1+d_{\phi}^{m}(z, \lambda)},
$$

which is bounded, by Lemma 6 .

(b) Given $v \in \ell_{(1+\varepsilon) \phi}^{p}(\Lambda)$, take $f=\sum_{\lambda} v_{\lambda} g_{\lambda}$ as before and estimate as in the proof of Lemma 34(d).

4.4. Inclusions between various spaces of sampling sequences. In this section we want to prove some inclusions between various spaces of sampling sequences. Unlike in the corresponding result for interpolating sequences, for the spaces of sampling sequences there is a gain, in the sense that any sampling sequence is actually sampling for a slightly bigger space. This will allow us to pass from the non-strict to the strict inequality of Theorem A.

A remark is in order. Given a sampling sequence, it is easy to "decrease" the space so that the sequence remains sampling. This can be proved as we did for interpolating sequences (and 
"increasing" spaces) in Theorem 35 (it is actually done in step (c) of our next theorem). It is more difficult, however, to slightly increase the space while keeping the sampling property of the sequence.

Theorem 36. Let $\Lambda \in \operatorname{Samp} \mathcal{F}_{\phi}^{p}$ be $\rho$-separated. There exists $\varepsilon>0$ such that for all $p^{\prime} \in[1, \infty]$ the sequence $\Lambda \in \operatorname{Samp} \mathcal{F}_{(1+\varepsilon) \phi}^{p^{\prime}}$.

Proof. The proof is divided in three steps.

(a) If $\Lambda \in \operatorname{Samp} \mathcal{F}_{\phi}^{p}$, then $\Lambda \in \operatorname{Samp} \mathcal{F}_{\phi}^{\infty}$. We know from Proposition 28 that for all weak limit $\left(\Lambda^{*}, \phi^{*}\right)$ the sequence $\Lambda^{*}$ is in Samp $\mathcal{F}_{\phi^{*}}^{p}$, and by Lemma 30 it will be enough to see that all weak limit $\Lambda^{*}$ is a uniqueness set for $\mathcal{F}_{\phi^{*}}^{\infty}$.

If this is not the case, there exists $f \in \mathcal{F}_{\phi^{*}}^{\infty}$ with $f \mid \Lambda^{*} \equiv 0, f \neq 0$.

We claim that for $m$ large enough

$$
g(z):=\frac{f(z)}{\left(z-\lambda_{1}^{*}\right) \ldots\left(z-\lambda_{m}^{*}\right)} \in \mathcal{F}_{\phi^{*}}^{p} .
$$

It is clear that Lemma 20 gives the $p$-integrability on $\cup_{j=1}^{m} D\left(\lambda_{j}^{*}\right)$. On the other hand, by Lemma 19

$$
\int_{z \notin \cup_{j} D\left(\lambda_{j}^{*}\right)} \frac{|f|^{p} e^{-p \phi^{*}} \rho_{\phi^{*}}^{-2}}{\left|z-\lambda_{1}^{*}\right|^{p} \ldots\left|z-\lambda_{m}^{*}\right|^{p}} \leq C \int_{z \notin \cup_{j} D\left(\lambda_{j}^{*}\right)} \frac{\|f\|_{\mathcal{F}_{\phi^{*}}}^{p} \Delta \phi^{*}}{\left|z-\lambda_{1}^{*}\right|^{p} \ldots\left|z-\lambda_{m}^{*}\right|^{p}} .
$$

Since $\Delta \phi^{*}$ is doubling there exists $m$ such that this integral converges (Lemma 5 (b)).

By Corollary 32, $\Lambda^{*} \backslash\left\{\lambda_{1}^{*} \ldots \lambda_{m}^{*}\right\} \in \operatorname{Samp} \mathcal{F}_{\phi^{*}}^{p}$. As $f$ vanishes on this sequence we deduce that $f \equiv 0$, which is a contradiction.

(b) If $\Lambda \in \operatorname{Samp} \mathcal{F}_{\phi}^{\infty}$ there exists $\varepsilon>0$ such that $\Lambda \in \operatorname{Samp} \mathcal{F}_{(1+\varepsilon) \phi}^{\infty}$. If this is not the case for any sequence $\left\{\varepsilon_{n}\right\} \searrow 0$ there exist functions $f_{n} \in \mathcal{F}_{\left(1+\varepsilon_{n}\right) \phi}^{\infty}$ and $\delta_{n}>0$ decreasing to 0 with $\left\|f_{n} \mid \Lambda\right\|_{\ell_{\left(1+\varepsilon_{n}\right) \phi}^{\infty}(\Lambda)} \leq \delta_{n}$ and $\left|f_{n}\left(z_{n}\right)\right|=1$.

Let $\Lambda_{n}=\tau_{z_{n}}^{-1}(\Lambda), \phi_{n}=\left(1+\varepsilon_{n}\right) \phi_{z_{n}}$ and $\tilde{f}_{n}=T_{z_{n}}^{\phi} f_{n}$. Then $\left|\tilde{f}_{n}(0)\right|=1,\left\|\tilde{f}_{n} \mid \Lambda_{n}\right\|_{\ell_{\phi_{n}}^{\infty}} \leq \delta_{n}$, and there exist a sequence $\Lambda^{*}$ and functions $\phi^{*}, f^{*}$ such that

$$
\left(\Lambda_{n}, \phi_{n}\right) \rightarrow\left(\Lambda^{*}, \phi^{*}\right) \in W(\Lambda, \phi)
$$

and $\left\{f_{n}\right\}_{n} \rightarrow f^{*} \in \mathcal{F}_{\phi^{*}}^{\infty}$ uniformly on compact sets. So we have $\left|f^{*}(0)\right|=1$ and $f^{*} \mid \Lambda^{*}=0$, i.e. $\Lambda^{*}$ is not a uniqueness sequence for $\mathcal{F}_{\phi^{*}}^{\infty}$, a contradiction with Lemma 30 .

(c) If $\Lambda \in \operatorname{Samp} \mathcal{F}_{(1+\varepsilon) \phi}^{\infty}$ for some $\varepsilon>0$, then $\Lambda \in \operatorname{Samp} \mathcal{F}_{\phi}^{p^{\prime}}$, for all $1 \leq p^{\prime} \leq \infty$. Consider the spaces

$$
\begin{aligned}
\mathcal{F}_{(1+\varepsilon) \phi}^{\infty, 0} & =\left\{f \in \mathcal{F}_{(1+\varepsilon) \phi}^{\infty}: \lim _{|z| \rightarrow \infty}|f(z)| e^{-(1+\varepsilon) \phi(z)}=0\right\}, \\
\ell_{(1+\varepsilon) \phi}^{\infty, 0}(\Lambda) & =\left\{v \in \ell_{(1+\varepsilon) \phi}^{\infty}: \lim _{|\lambda| \rightarrow \infty}\left|v_{\lambda}\right| e^{-(1+\varepsilon) \phi(\lambda)}=0\right\} .
\end{aligned}
$$


There is a sequence of functions $\{g(z, \lambda)\}_{\lambda \in \Lambda}$ such that for all $f \in \mathcal{F}_{(1+\varepsilon) \phi}^{\infty, 0}$

$$
e^{-(1+\varepsilon) \phi(z)} f(z)=\sum_{\lambda \in \Lambda} e^{-(1+\varepsilon) \phi(\lambda)} f(\lambda) g(z, \lambda)
$$

and $\sum_{\lambda}|g(z, \lambda)| \leq K$ uniformly in $z$. This is so by a duality argument, because

$$
\{f(\lambda)\}_{\lambda \in \Lambda} \mapsto e^{-(1+\varepsilon) \phi(z)} f(z) \quad \text { with } f \in \mathcal{F}_{(1+\varepsilon) \phi}^{\infty, 0}
$$

is a bounded linear functional from a closed subspace of $\ell_{(1+\varepsilon) \phi}^{\infty, 0}(\Lambda)$ whose norm is bounded independently of $z$. This is an argument from [Beu89, pp. 348-358] (see also [Sei93, p.36]).

Consider now $f \in \mathcal{F}_{\phi}^{p} \subset \mathcal{F}_{\phi}^{\infty, 0}$. Given $z \in \mathbb{C}$ take the function $P_{z}$ of Theorem 18 for $\varepsilon>0$. Then $f P_{z} \in \mathcal{F}_{(1+\varepsilon) \phi}^{\infty, 0}$, and by the representation above

$$
e^{-(1+\varepsilon) \phi(z)} f(z)=\sum_{\lambda \in \Lambda} e^{-(1+\varepsilon) \phi(\lambda)} f(\lambda) P_{z}(\lambda) g(z, \lambda) .
$$

Hence

$$
\begin{aligned}
|f(z)| e^{-\phi(z)} & \lesssim \sum_{\lambda \in \Lambda}|f(\lambda)| e^{-\phi(\lambda)}\left|P_{z}(\lambda)\right| e^{\varepsilon(\phi(z)-\phi(\lambda))}|g(z, \lambda)| \\
& \lesssim \sum_{\lambda \in \Lambda}|f(\lambda)| e^{-\phi(\lambda)} \frac{|g(z, \lambda)|}{1+d_{\phi}^{m}(z, \lambda)} .
\end{aligned}
$$

The case $p=\infty$ is clear, so assume that $p<\infty$. Since $\sum_{\lambda}|g(z, \lambda)| \leq K$, we may apply Jensen's inequality and obtain

$$
\rho^{-2}(z)|f(z)|^{p} e^{-p \phi(z)} \lesssim \rho^{-2}(z) \sum_{\lambda \in \Lambda}|f(\lambda)|^{p} e^{-p \phi(\lambda)} \frac{|g(z, \lambda)|}{1+d_{\phi}^{m p}(z, \lambda)} .
$$

Now integrate, use that $|g(z, \lambda)| \leq K$ and apply Lemma 5(b) to finally obtain the sampling inequality

$$
\int_{\mathbb{C}}|f(z)|^{p} e^{-p \phi(z)} d \sigma(z) \lesssim \sum_{\lambda \in \Lambda}|f(\lambda)|^{p} e^{-p \phi(\lambda)}
$$

4.5. Nets. We finish this section by giving useful examples of interpolating and sampling sequences.

Lemma 37. Let $f$ be the multiplier associated to $\phi$, as constructed in the proof of Theorem 17 and let $\Lambda=\mathcal{Z}(f)$. Then $\mathcal{D}_{\Delta \phi}^{+}(\Lambda)=\mathcal{D}_{\Delta \phi}^{-}(\Lambda)=1 / 2 \pi$. We say that $\Lambda$ is a net associated to $\phi$. 
Proof. The construction of $f$ is made with quasi-squares $R_{p}$ of $\mu\left(R_{p}\right)=2 \pi m N$ and $m N$ associated points in a dilated $C R_{p}$ that made up $\Lambda$. Thus, for $z \in \mathbb{C}$ and $t>0$ :

$$
\begin{aligned}
& n(z, t \rho(z)) \geq m N \#\left\{p: C R_{p} \subset D^{t}(z)\right\}=\frac{1}{2 \pi} \mu\left(\bigcup_{p: C R_{p} \subset D^{t}(z)} R_{p}\right), \\
& n(z, t \rho(z)) \leq m N \#\left\{p: C R_{p} \cap D^{t}(z) \neq \emptyset\right\}=\frac{1}{2 \pi} \mu\left(\bigcup_{p: C R_{p} \cap D^{t}(z) \neq \emptyset} R_{p}\right) .
\end{aligned}
$$

By Corollary 10,

$$
D^{t-\epsilon(t)}(z) \subset \bigcup_{p: C R_{p} \subset D^{t}(z)} R_{p} \subset D^{t}(z) \subset \bigcup_{p: C R_{p} \cap D^{t}(z) \neq \emptyset} R_{p} \subset D^{t+\epsilon(t)}(z),
$$

whence

$$
\frac{1}{2 \pi} \mu\left(D^{t-\epsilon(t)}(z)\right) \leq n(z, t \rho(z)) \leq \frac{1}{2 \pi} \mu\left(D^{t+\epsilon(t)}(z)\right) .
$$

The result is then an application of Lemma 11 .

Lemma 38. Let $\Lambda$ be a net associated to $\phi$. Then $\Lambda \in \operatorname{Int} \mathcal{F}_{(1+\varepsilon) \phi}^{p}$ and $\Lambda \in \operatorname{Samp} \mathcal{F}_{(1-\varepsilon) \phi}^{p}$ for all $\varepsilon>0$ and $1 \leq p \leq \infty$.

Proof. Let $f$ be a multiplier associated to $\phi$ such that $\Lambda=\mathcal{Z}(f)$.

Let us start by proving that $\Lambda$ is interpolating. By Theorem 35 it is enough to prove that $\Lambda \in \operatorname{Int} \mathcal{F}_{(1+\varepsilon) \phi}^{\infty}$ for all $\varepsilon>0$.

For each $\lambda \in \Lambda$ define

$$
g_{\lambda}(z)=\frac{f(z)}{z-\lambda} \frac{1}{f^{\prime}(\lambda)} .
$$

Clearly $g_{\lambda}\left(\lambda^{\prime}\right)=\delta_{\lambda}^{\lambda^{\prime}}$. The growth condition of the multiplier gives $\left|f^{\prime}(\lambda)\right| \simeq e^{\phi(\lambda)} / \rho(\lambda)$. This and Lemma 4 show that for some $\gamma>0$,

$$
\left|g_{\lambda}(z)\right| e^{-\phi(z)} \lesssim e^{-\phi(\lambda)} \frac{d_{\phi}(z, \Lambda)}{|z-\lambda|} \rho(\lambda) .
$$

When $z \in D(\lambda)$ it is clear that this is bounded by $e^{-\phi(\lambda)}$, by Lemma 4(a). The same bound holds for $z \notin D(\lambda)$, using Lemma 4 (b) together with the fact that $d_{\phi}(z, \Lambda) \lesssim 1$.

As seen in the proof of Theorem 35 the existence of such $g_{\lambda}$ is enough to construct, for any $\varepsilon>0$, an interpolation operator for $\overline{\mathcal{F}}_{(1+\varepsilon) \phi}^{p}$.

Let us see next that $\Lambda \in \operatorname{Samp} \mathcal{F}_{(1-\varepsilon) \phi}^{p}$. By Theorem 36 it is enough to consider the case $p=\infty$, and by Corollary 30 it will be enough to see that every weak limit $\left(\Lambda^{*},(1-\varepsilon) \phi^{*}\right) \in$ $W(\Lambda,(1-\varepsilon) \phi)$ is a uniqueness sequence for $\mathcal{F}_{(1-\varepsilon) \phi^{*}}^{\infty}$.

Let $\left(\Lambda_{z_{n}}, \phi_{z_{n}}\right) \rightarrow\left(\Lambda^{*}, \phi^{*}\right)$ and let $f_{z_{n}}$ be the corresponding multipliers, with $\mathcal{Z}\left(f_{z_{n}}\right)=\Lambda_{z_{n}}$ and $\left|f_{z_{n}}(z)\right| \simeq e^{\phi_{z_{n}}(z)} d_{\phi_{z_{n}}}\left(z, \Lambda_{z_{n}}\right)$. By Montel's theorem let $\left\{f_{z_{n}}\right\}_{n} \rightarrow f^{*}$ with $\mathcal{Z}\left(f^{*}\right)=\Lambda^{*}$ and $\left|f^{*}(z)\right| \simeq e^{\phi^{*}(z)} d_{\phi^{*}}\left(z, \Lambda^{*}\right)$, i.e, $f^{*}$ is a multiplier for $\phi^{*}$. 
Consider also a multiplier $g$ associated to $\varepsilon \phi^{*}$. In particular $|g(z)| \simeq e^{\varepsilon \phi^{*}(z)} d_{\phi^{*}}(z, \mathcal{Z}(g))$. In order to see that $\Lambda^{*}$ is a uniqueness sequence assume that $h \in \mathcal{F}_{(1-\varepsilon) \phi^{*}}^{\infty}$ and $h \mid \Lambda^{*}=0$. Then $h g \in \mathcal{F}_{\phi^{*}}^{\infty}$, by construction. On the other hand, the function $F:=h g / f^{*}$ is entire, because $h$ vanishes on $\Lambda^{*}$. It is also bounded when $z$ is far from $\Lambda^{*}$, since $|h g| \lesssim e^{\phi^{*}}$ and $\left|f^{*}\right| \gtrsim e^{\phi^{*}}$. By the maximum principle $F$ is bounded globally, and by Liouville's theorem there exists $c \in \mathbb{C}$ such that $h g=c f^{*}$. Since $g$ vanishes in some points outside $\Lambda^{*}$ we have $c=0$, hence $h \equiv 0$.

\section{SUFFICIENT CONDITIONS FOR SAMPLING}

We prove here the sufficiency part of Theorem A. We will use the same technique as in [Beu89, pp. 341-350]. Assume that $\mathcal{D}_{\Delta \phi}^{-}(\Lambda)>1 / 2 \pi$. By Lemma 27 we can assume that $\Lambda$ is $\rho$-separated, and according to Theorem 36 it will be enough to prove that $\Lambda \in \operatorname{Samp} \mathcal{F}_{\phi}^{\infty}$. By Corollary 30 this will be done as soon as we show that every weak limit $\Lambda^{*}$ is a uniqueness sequence for $\mathcal{F}_{\phi^{*}}^{\infty}$.

Recall the notation $n_{\Lambda}(z, r)=\#[\Lambda \cap \overline{D(z, r)}]$.

Assume thus that we have $f \in \mathcal{F}_{\phi^{*}}^{\infty}$ with $f \mid \Lambda^{*} \equiv 0$ and $\|f\|_{\mathcal{F}_{\phi^{*}}^{\infty}}=1$. There is no loss of generality in assuming that $f(0) \neq 0$. Applying Jensen's formula to $f$ on $D_{\phi^{*}}(0)$

$$
\begin{aligned}
\int_{0}^{r \rho_{\phi^{*}}(0)} \frac{n_{\Lambda^{*}}(0, t)}{t} d t & =\frac{1}{2 \pi} \int_{0}^{2 \pi} \log \left|f\left(r \rho_{\phi^{*}}(0) e^{i \theta}\right)\right| d \theta-\log |f(0)| \\
& \leq \frac{1}{2 \pi} \int_{0}^{2 \pi} \phi^{*}\left(r \rho_{\phi^{*}}(0) e^{i \theta}\right) d \theta-\log |f(0)| \\
& =\left[\frac{1}{2 \pi} \int_{0}^{2 \pi} \phi^{*}\left(r \rho_{\phi^{*}}(0) e^{i \theta}\right) d \theta-\phi^{*}(0)\right]+\phi^{*}(0)-\log |f(0)| .
\end{aligned}
$$

By Lemma 4, Then, Green's identity yields

$$
\begin{aligned}
\int_{0}^{r \rho_{\phi^{*}}(0)} \frac{n_{\Lambda^{*}}(0, t)}{t} d t & \leq \frac{1}{2 \pi} \int_{D\left(0, r \rho_{\phi^{*}}(0)\right)} \log \frac{r \rho_{\phi^{*}}(0)}{|\zeta|} \Delta \phi^{*}(\zeta)+\mathrm{O}(1) \\
& =\frac{1}{2 \pi} \int_{0}^{r \rho_{\phi^{*}}(0)} \Delta \phi^{*}(D(0, t)) \frac{d t}{t}+\mathrm{O}(1),
\end{aligned}
$$

for all $r$ big enough. This contradicts the hypothesis, which implies in particular that for some $\varepsilon>0$ and all $t$ big enough $n_{\Lambda^{*}}(0, t) \geq(1 / 2 \pi+\varepsilon) \Delta \phi^{*}(D(0, t))$.

\section{NECESSARY CONDITIONS FOR SAMPLING}

This section contains the proof of the necessity part of Theorem A. By Lemma 27(b) and Theorem 36 it will be enough to prove the following result.

Theorem 39. Let $\Lambda$ be $\rho$-separated. If $\Lambda \in \operatorname{Samp} \mathcal{F}_{\phi}^{2}$ then $\mathcal{D}_{\Delta \phi}^{-}(\Lambda) \geq 1 / 2 \pi$.

We use a result comparing the densities between interpolating and sampling sequences, as in [RS95]. We do that by adapting Lemma 4 in [OCS98] to our setting. 
Lemma 40. Let $\varepsilon>0$. Assume $I \in \operatorname{Int} \mathcal{F}_{(1-\varepsilon) \phi}^{2}$ and $S \in \operatorname{Samp} \mathcal{F}_{\phi}^{2}$ is $\rho$-separated. There exists a positive function $\epsilon(t)$ such that $\lim _{t \rightarrow \infty} \epsilon(t) / t=0$ and for every $\epsilon>0$ there is $t_{0}>0$ with

$$
(1-\epsilon) n_{I}(z, t \rho(z)) \leq n_{S}(z,(t+\epsilon(t)) \rho(z)), \quad z \in \mathbb{C}, \forall t \geq t_{0} .
$$

Proof. The proof is as in [OCS98, Lemma 4] with minor modifications, so we keep it short.

According to our definition, if $S$ is sampling then $\left\{k(z, s)=K_{\phi}(z, s) e^{-\phi(s)}\right\}_{s \in S}$ is a frame in $\mathcal{F}_{\phi}^{2}\left(K_{\phi}\right.$ denotes the Bergman kernel, as in Section 3.3). That is, for $f \in \mathcal{F}_{\phi}^{2}$

$$
\|f\|_{\mathcal{F}_{\phi}^{2}}^{2} \simeq \sum_{s \in S}|\langle k(z, s), f(z)\rangle|^{2}
$$

A consequence is that

$$
f(z)=\sum_{s \in S}\langle k(\xi, s), f(\xi)\rangle \tilde{k}(z, s)=\sum_{s \in S} f(s) e^{-\phi(s)} \tilde{k}(z, s),
$$

where $\tilde{k}(z, s)$ is the dual frame of $k(z, s)$.

Consider also the functions $g_{i}$ given by Lemma 34 for the weight $(1-\varepsilon) \phi$. Lemma $34(\mathrm{~d})$ implies that the normalised functions $\kappa(i, z):=g_{i}(z) e^{\phi(i)}$ form a Riesz basis in the closed linear span $H$ of $\{\kappa(i, z)\}_{i \in I}$ in $\mathcal{F}_{\phi}^{2}$.

Given $z \in \mathbb{C}$ and $t, r>0$ ( $t$ much bigger that $r$ ) consider the following two finite dimensional subspaces of $\mathcal{F}_{\phi}^{2}$ :

$$
\begin{aligned}
& W_{S}=<\tilde{k}(\xi, s): s \in S \cap D^{t+r}(z)> \\
& W_{I}=<\kappa(\xi, i): i \in I \cap D^{t}(z)>.
\end{aligned}
$$

Let $P_{S}$ and $P_{I}$ denote the orthogonal projections of $\mathcal{F}_{\phi}^{2}$ on $W_{S}$ and $W_{I}$ respectively. We estimate the trace of the operator $T=P_{I} P_{S}$ in two different ways. To begin with

$$
\operatorname{tr}(T) \leq \operatorname{rank} W_{S} \leq \#\left\{S \cap D^{t+r}(z)\right\} .
$$

On the other hand

$$
\operatorname{tr}(T)=\sum_{i \in I \cap D^{t}(z)}\left\langle T(\kappa(\xi, i)), P_{I} \kappa^{*}(\xi, i)\right\rangle
$$

where $\left\{\kappa^{*}(\xi, i)\right\}$ is the dual basis of $\kappa(\xi, i)$ in $H$. Using that $P_{I}$ and $P_{S}$ are projections one deduces that

$$
\operatorname{tr}(T) \geq \#\left\{i \in I \cap D^{t}(z)\right\}\left(1-\sup _{i}\left|\left\langle P_{S}(\kappa(\xi, i))-\kappa(\xi, i), \kappa^{*}(\xi, i)\right\rangle\right|\right) .
$$

Since $\|\kappa(\xi, i)\|_{\mathcal{F}_{\phi}^{2}} \simeq 1$, also $\left\|\kappa^{*}(\xi, i)\right\|_{\mathcal{F}_{\phi}^{2}} \simeq 1$. Thus we will be done as soon as we show that $\left\|P_{S}(\kappa(\xi, i))-\kappa(\xi, i)\right\|_{\mathcal{F}_{\phi}^{2}} \leq \varepsilon$ for a suitable $r \simeq \epsilon(t)$.

We have

$$
\left\|P_{S}(\kappa(\xi, i))-\kappa(\xi, i)\right\|_{\mathcal{F}_{\phi}^{2}}^{2} \lesssim \sum_{s \notin D^{t+r}(z)}|\langle k(\xi, s), \kappa(\xi, i)\rangle|^{2}=\sum_{s \notin D^{t+r}(z)}\left|\kappa(s, i) e^{-\phi(s)}\right|^{2} .
$$


Since $S$ is $\rho$-separated, there exists $\eta>0$ such that the disks $D^{\eta}(s)$ are pairwise disjoint. Using Lemma 19.(a) we get, for some $c>0$ depending on $\phi$ and $\eta$

$$
\left\|P_{S}(\kappa(\xi, i))-\kappa(\xi, i)\right\|_{\mathcal{F}_{\phi}^{2}}^{2} \lesssim \bigcup_{s \notin D^{t+r}(z)} \int_{D^{\eta}(s)}|\kappa(\zeta, i)|^{2} e^{-2 \phi(\zeta)} d \sigma(\zeta) .
$$

Applying Lemma 9 with $r^{k}=t^{\tau}$ and $\tau$ so that $0<(\varepsilon-\tau) \gamma<1$, we see that there exist $\delta \in(0,1), c>0$ and a function $\epsilon(t)=c t^{1-\delta}$ such that

$$
\bigcup_{s \notin D^{t+c \epsilon(t)}(z)} D^{\eta}(s) \subset \mathbb{C} \backslash D^{\varepsilon \epsilon(t)}(i) .
$$

Finally, for $t$ big enough Lemma 34(e) yields

$$
\left\|P_{S}(\kappa(\xi, i))-\kappa(\xi, i)\right\|_{\mathcal{F}_{\phi}^{2}}^{2} \lesssim \int_{\mathbb{C} \backslash D^{\epsilon \epsilon(t)}(i)}|\kappa(\xi, i)|^{2} e^{-2 \phi(\xi)} d \sigma(\xi) \lesssim \epsilon
$$

Proof of Theorem 39. Given $\varepsilon>0$ consider a net $I$ associated to $(1-2 \varepsilon) \phi$. By Lemma 37 $I \in \operatorname{Int} \mathcal{F}_{(1-\varepsilon) \phi}^{2}$, and by Lemma $38 \mathcal{D}_{\Delta \phi}^{+}(I)=\mathcal{D}_{\Delta \phi}^{+}(I)=(1-2 \varepsilon) / 2 \pi$. Apply now Lemma 40 ; there exist $t_{0}$ and $\epsilon(t)$ such that for $t>t_{0}$

$$
n_{\Lambda}(z, t \rho(z)) \geq(1-\varepsilon) n_{I}(z,(t-\epsilon(t)) \rho(z)) \geq \frac{(1-\varepsilon)^{3}}{2 \pi} \mu\left(D^{t-\delta(t)}(z)\right),
$$

where $\delta(t)=t-\epsilon(t)-\epsilon(t-\epsilon(t))$. This estimate together with Lemma 11 finish the proof.

\section{SUFFICIENT CONDITIONS FOR INTERPOLATION}

Taking into account Lemma 38, in order to prove the sufficiency part of Theorem B it is enough to prove the following.

Theorem 41. If $\Lambda$ is $\rho$-separated and $\mathcal{D}_{\Delta \phi}^{+}(\Lambda)<1 / 2 \pi$ there exist $\varepsilon>0$ and a sequence $\Sigma$ such that $\Lambda \cup \Sigma$ is a $\rho$-separated net associated to $(1-\varepsilon) \phi$.

In the proof of this result we need to express the density condition in terms of the quasi-squares appearing in Theorem 8 , this will be done in Theorem 43, before we need some preliminaries.

Denote $\phi_{r}=e^{-r} \phi$.

Lemma 42. Let

$$
I_{r}(\zeta)=\int_{|z-\zeta|<\rho_{\phi_{r}}(z) / r} \frac{r^{2} d m(z)}{\pi \rho_{\phi_{r}}^{2}(z)}
$$

Then $\sup _{\zeta \in \mathbb{C}}\left|I_{r}(\zeta)-1\right|<1 / r$. 
Proof. We estimate $I_{r}$ using the change of variable $w=(z-\zeta) / \rho_{\phi_{r}}(z)$, whose Jacobian is

$$
\rho_{\phi_{r}}^{-2}(z)\left|1-\frac{\left\langle\nabla \rho_{\phi_{r}}(z), z-\zeta\right\rangle}{\rho_{\phi_{r}}(z)}\right| \text {. }
$$

From (6) it follows that $\left|\nabla \rho_{\phi_{r}}\right| \leq 1$, hence the Jacobian is bounded above by $\rho_{\phi_{r}}^{-2}(z)(1+1 / r)$ and below by $\rho_{\phi_{r}}^{-2}(z)(1-1 / r)$. Then

$$
1-\frac{1}{r} \int_{|w| \leq 1 / r} \frac{r^{2}}{\pi}\left(1-\frac{1}{r}\right) d m(w) \leq I_{r}(\zeta) \leq \int_{|w| \leq 1 / r} \frac{r^{2}}{\pi}\left(1+\frac{1}{r}\right) d m(w)=1+\frac{1}{r} .
$$

Using (9) with $t=e^{r}$ we have

$$
\lim _{r \rightarrow \infty} \frac{\rho_{\phi_{r}}(z)}{r \rho_{\phi}(z)}=\infty
$$

uniformly in $z \in \mathbb{C}$.

Let $R_{\alpha}^{s}(z)$ denote the rectangle with vertices $z+s \rho(z)(1+i \alpha), z+s \rho(z)(1-i \alpha), z-s \rho(z)(1+$ $i \alpha)$ and $z-s \rho(z)(1-i \alpha)$, where $\alpha \in\left[e^{-1}, e\right]$ and $e$ is the constant of Theorem $8(\mathrm{~b})$.

Theorem 43. Let $\mu=\Delta \phi$ and let $\nu$ be a positive measure such that

$$
\nu\left(D_{\phi}^{r}(z)\right) \leq(1-\varepsilon) \mu\left(D_{\phi}^{r}(z)\right) \quad \forall r \geq r_{0}, \forall z \in \mathbb{C} .
$$

There exists $s_{0}>0$ such that for any $\alpha \in\left[e^{-1}, e\right]$

$$
\nu\left(R_{\alpha}^{s}(z)\right) \leq\left(1-\frac{\varepsilon}{2}\right) \mu\left(R_{\alpha}^{s}(z)\right) \quad \forall s \geq s_{0}, \forall z \in \mathbb{C} .
$$

Proof. Fix $r$ big enough so that $\rho_{\phi_{r}} / r>r_{0} \rho_{\phi}$ and $(1+1 / r)(1-\varepsilon)<(1-1 / r)(1-3 \varepsilon / 4)$. This can be done because of (16). By hypothesis

$$
\nu\left(D_{\phi_{r}}^{1 / r}(z)\right) \leq(1-\varepsilon) \mu\left(D_{\phi_{r}}^{1 / r}(z)\right) \quad \forall z \in \mathbb{C},
$$

and if $s$ is much bigger than $r$ we get

$$
\int_{z \in R_{s}^{\alpha}(w)} \frac{r^{2}}{\pi \rho_{\phi_{r}}^{2}(z)} \nu\left(D_{\phi_{r}}^{1 / r}(z)\right) d m(z) \leq(1-\varepsilon) \int_{z \in R_{s}^{\alpha}(w)} \frac{r^{2}}{\pi \rho_{\phi_{r}}^{2}(z)} \mu\left(D_{\phi_{r}}^{1 / r}(z)\right) d m(z) .
$$

Denote

$$
\begin{aligned}
\Omega_{r}(\zeta) & =\left\{z \in \mathbb{C},|z-\zeta|<\rho_{\phi_{r}}(z) / r\right\} \\
F_{r}(w, s) & =\left\{\zeta \in \mathbb{C}, \Omega_{r}(\zeta) \subset R_{\alpha}^{s}(w)\right\} \\
G_{r}(w, s) & =\bigcup_{\zeta \in R_{\alpha}^{s}(w)} \Omega_{r}(\zeta) .
\end{aligned}
$$

Reversing the order of integration and using the previous lemma we deduce that

$$
\nu\left(F_{r}(w, s)\right) \leq\left(1-\frac{3}{4} \varepsilon\right) \mu\left(G_{r}(w, s)\right) .
$$


It is clear that $F_{r}(w, s) \subset R_{\alpha}^{s}(w) \subset G_{r}(w, s)$. Similarly to the proof of Lemma 9, there exists $\epsilon(s)$ such that $R_{\alpha}^{s-\epsilon(s)}(w) \subset F_{r}(w, s)$ and $G_{r}(w, s) \subset R_{\alpha}^{s+\epsilon(s)}(w)$.

By Remark 4

$$
\lim _{s \rightarrow \infty} \frac{\mu\left(R_{\alpha}^{s+\epsilon(s)}(w)\right)}{\mu\left(R_{\alpha}^{s-\epsilon(s)}(w)\right)}=1
$$

uniformly in $z$, and therefore there exists $s_{0}$ such that for $s>s_{0}$

$$
\begin{aligned}
\nu\left(R_{\alpha}^{s-\epsilon(s)}(w)\right) & \leq\left(1-\frac{3}{4} \varepsilon\right) \mu\left(G_{r}(w, s)\right) \leq\left(1-\frac{3}{4} \varepsilon\right) \mu\left(R_{\alpha}^{s+\epsilon(s)}(w)\right) \\
& \leq\left(1-\frac{\varepsilon}{2}\right) \mu\left(R_{\alpha}^{s-\epsilon(s)}(w)\right) .
\end{aligned}
$$

Proof of Theorem 41. Take an entire function $g$ vanishing exactly on $\Lambda$. We will construct a sequence $\Sigma$ and an entire function $h$ such that for some $\varepsilon>0$,

(i) $\Lambda \cup \Sigma$ is $\rho$-separated.

(ii) $h$ vanishes exactly on $\Sigma$.

(iii) For any $\tau>0,|\log | h(z)|-(1-\varepsilon) \phi(z)+\log | g(z)|| \leq C_{\tau}$ if $D^{\tau}(z) \cap(\Lambda \cup \Sigma)=\emptyset$.

Accepting this we reach the result by taking $f=g h$. This is so because the separateness of $\Lambda \cup \Sigma$ and (iii) imply that $f$ is a multiplier for $(1-\varepsilon) \phi$.

Construction of $\Sigma$ and $h$. To avoid the repetition of the factors $2 \pi$ and $1-\varepsilon$, denote here $\mu=$ $(1-\varepsilon) \Delta \phi / 2 \pi$. Let

$$
\tilde{\mu}=\mu-\sum_{\lambda \in \Lambda} \delta_{\lambda}=\frac{1}{2 \pi} \Delta((1-\varepsilon) \phi-\log |g|) .
$$

Following Theorem 8 and the Remark thereafter, given $n, M \in \mathbb{N}$ we can take a system of quasisquares $\left\{R_{k}\right\}_{k}$ such that, denoting $\mu_{k}=\mu_{\mid R_{k}}$, we have $\mu=\sum_{k} \mu_{k}$ and $\mu_{k}(\mathbb{C})=M n$. Then $\tilde{\mu}=\sum_{k} \tilde{\mu}_{k}$, being

$$
\tilde{\mu}_{k}=\mu_{k}-\sum_{\lambda \in \Lambda \cap R_{k}} \delta_{\lambda}
$$

By hypothesis there exists $\varepsilon>0$ such that $\mathcal{D}_{\Delta \phi}(\Lambda)<1 / 2 \pi-4 \varepsilon$. Therefore, there exists $r_{0}>0$ such that

$$
\tilde{\mu}\left(D^{r}(z)\right) \geq 3 \varepsilon \mu\left(D^{r}(z)\right) \quad \text { for all } \quad z \in \mathbb{C}, r \geq r_{0} .
$$

Also, Theorem 43 implies that for $M \geq m /(2 \varepsilon)$ and $n$ big enough:

$$
M n \geq \tilde{\mu}\left(R_{k}\right) \geq 2 \varepsilon \mu\left(R_{k}\right)=2 \varepsilon M n \geq m n .
$$

Let $\tilde{\mu}\left(R_{k}\right)=m_{k} n$, with $m \leq m_{k} \leq M$. Notice that $m_{k} \in \mathbb{N}$, since $\mu\left(R_{k}\right) \in \mathbb{N}$. Applying Lemma 15 we obtain a sequence $\Sigma$ made of points $\sigma_{1}^{k}, \ldots, \sigma_{m_{k} n}^{k} \in C R_{k}$ so that the first $m$ moments of the measures $\nu_{k}=\tilde{\mu}_{k}-\sum_{j=1}^{m_{k} n} \delta_{\sigma_{j}^{k}}$ vanish. Furthermore, it is clear that we can choose the $\tau_{j}^{k}$ in the proof of Lemma 15 so that $\Lambda \cup \Sigma$ is $\rho$-separated. 
Let

$$
\nu=\sum_{k} \nu_{k}=\frac{1}{2 \pi} \Delta((1-\varepsilon) \phi-\log |g|)-\sum_{\sigma \in \Sigma} \delta_{\sigma} .
$$

In order to prove (iii) consider $v=(1-\varepsilon) \phi-\log |g|-w$, where

$$
w(z)=\int_{\mathbb{C}} \log |z-\zeta| d \nu(\zeta) .
$$

Since

$$
\Delta v=2 \pi \sum_{\sigma \in \Sigma} \delta_{\sigma}
$$

there exists $h$ entire (vanishing exactly on $\Sigma$ ) such that $\log |h|=v$.

We need to estimate $|w(z)|$ when $|z-\Lambda \cup \Sigma| \geq \tau \rho(z)$. Given $z \in \mathbb{C}$, let $k_{0} \in \mathbb{N}$ be such that $z \in R_{k_{0}}$. By Theorem 8 (c), there exists $r_{0}>0$ independent of $z$ such that $R_{k_{0}} \subset D^{r_{0}}(z) \subset$ $C R_{k_{0}}$. We have

$$
w(z)=\int_{\mathbb{C}} \log |z-\zeta| d \nu(\zeta)=\int_{\mathbb{C}} \log |z-\zeta| d \nu_{k_{0}}(\zeta)+\sum_{k: k \neq k_{0}} \int_{\mathbb{C}} \log |z-\zeta| d \nu_{k}(\zeta),
$$

and we estimate the two terms separately.

Let $C>0$ be the constant of Lemma 15 . Since the first $m$ moments of $\nu_{k_{0}}$ vanish,

$$
\begin{aligned}
\left|\int_{\mathbb{C}} \log \right| z-\zeta\left|d \nu_{k_{0}}(\zeta)\right| & =\left|\int_{\mathbb{C}} \log \frac{|z-\zeta|}{r_{0} \rho(z)} d \nu_{k_{0}}(\zeta)\right| \lesssim\left|\int_{C R_{k_{0}}} \log \frac{r_{0} \rho(z)}{|z-\zeta|} d \mu(\zeta)\right|+K|\log \tau| \\
& \lesssim \int_{D^{c r_{0}}(z)} \log \frac{c r_{0} \rho(z)}{|z-\zeta|} d \mu(\zeta)+K|\log \tau| \leq C_{\tau} .
\end{aligned}
$$

The other integral is estimated using the moment condition for each $\nu_{k}$, as in the estimate of $I_{1}$ in Theorem 14 .

\section{NECESSARY CONDITIONS FOR INTERPOLATION}

Let us start by proving the non-strict density inequality. By Theorem 35, it is enough to consider the case $p=2$.

Theorem 44. If $\Lambda \in \operatorname{Int} \mathcal{F}_{\phi}^{2}$ then $\mathcal{D}_{\Delta \phi}^{+}(\Lambda) \leq 1 / 2 \pi$.

Proof. Given $\varepsilon>0$, take a net $S$ associated to $(1+2 \varepsilon) \phi$, as described in Lemma 37. Lemma 38 implies that $S \in \operatorname{Samp} \mathcal{F}_{(1+\varepsilon) \phi}^{2}$, and by Lemma 40, there exists $t_{0}>0$ such that

$$
n_{\Lambda}(z, t \rho(z)) \leq(1+\varepsilon) n_{S}(z,(t+\epsilon(t)) \rho(z)) \quad z \in \mathbb{C}, t \geq t_{0} .
$$

Since $S$ is a net of density $(1+2 \varepsilon) / 2 \pi$, the radius $t_{0}$ can be taken so that for $t \geq t_{0}$

$$
n_{S}(z,(t+\epsilon(t)) \rho(z)) \leq \frac{1+3 \varepsilon}{2 \pi} \mu\left(D^{t+\epsilon(t)}(z)\right) .
$$

This and Corollary 10 give the result. 
Let us see now that the inequality is strict.

Proof of the necessity part in Theorem B. Assume that $\Lambda \in \operatorname{Int} \mathcal{F}_{\phi}^{p}$. We know that $\mathcal{D}_{\Delta \phi}^{+}(\Lambda) \leq$ $1 / 2 \pi$. In order to see that $\mathcal{D}_{\Delta \phi}^{+}(\Lambda)<1 / 2 \pi$ consider, given $\varepsilon>0$, a net $\Sigma$ associated to $2 \varepsilon \phi$ such that $Z:=\Lambda \cup \Sigma$ is $\rho$-separated.

Lemma 45. Denote $Z=\left\{z_{k}\right\}_{k}$. For every $m \in \mathbb{N}$ and $\varepsilon>0$ there exist $C>0$ and functions $f_{k} \in \mathcal{F}_{\phi}^{\infty}$ such that

(a) $f_{k}\left(z_{k}\right)=1$.

(b) $f_{k}\left(z_{j}\right)=0$ for all $z_{j} \in D^{1 / \varepsilon}\left(z_{k}\right)$.

(c) $\left|f_{k}(z)\right| \leq C M(\Lambda) e^{\phi(z)-\phi\left(z_{k}\right)} \frac{1}{1+d_{\phi}^{m}\left(z, z_{k}\right)}$.

(d) $\left\|f_{k}\right\|_{\phi, \infty} \leq C M(\Lambda) e^{-\phi\left(z_{k}\right)}$.

Proof. Assume first that $z_{k}=\lambda_{k} \in \Lambda$. By hypothesis there exists $g_{k} \in \mathcal{F}_{\phi}^{p} \subset \mathcal{F}_{\phi}^{\infty}$ with $g_{k}\left(\lambda_{k}\right)=1, g\left(\lambda_{j}\right)=0$, and $\left\|g_{k}\right\|_{\mathcal{F}_{\phi}^{\infty}} \leq M(\Lambda) e^{-\phi\left(\lambda_{k}\right)}$. Since $\Lambda$ plus a finite number of points is still in Int $\mathcal{F}_{\phi}^{p}$ (Lemma 33, we can take $g_{k}$ so that moreover $g_{k}\left(\sigma_{j}\right)=0$ if $\left|\lambda_{k}-\sigma_{j}\right| \leq 1 / \varepsilon \rho\left(\lambda_{k}\right)$ and $g_{k}\left(c_{j}\right)=0, j=1, \ldots, M$, where $c_{j}=\lambda_{k}+2 \delta \rho\left(\lambda_{k}\right) e^{j \frac{2 \pi i}{M}}$ and $\delta>0$ is taken so that the balls $\{B(\lambda, 10 \delta)\}_{\lambda}$ are pairwise disjoint.

By construction of the nets there exists $C$ independent of $z$ and $\varepsilon$ such that $\# \Sigma \cap D^{1 / \varepsilon}(z) \leq C$ for any $\Sigma$ net of density $\varepsilon / \pi$.

Define then

$$
f_{k}(z)=(2 \delta)^{-M} \frac{g_{k}(z)}{\left(z-c_{1}\right) \cdots\left(z-c_{M}\right)} \rho^{M}\left(z_{k}\right) .
$$

It is clear that $f_{k} \in \mathcal{F}_{\phi}^{\infty}$ satisfies (a) and (b).

For $z \notin \cup_{j=1}^{M} D^{\delta}\left(c_{j}\right)$,

$$
\left|f_{k}(z)\right| \leq C\left|g_{k}(z)\right|\left(\frac{\rho\left(z_{k}\right)}{\left|z-z_{k}\right|}\right)^{M} \leq C M(\Lambda) e^{\phi(z)-\phi\left(z_{k}\right)}\left(\frac{\rho\left(z_{k}\right)}{\left|z-z_{k}\right|}\right)^{M},
$$

and the estimate follows from Lemma 4.

For $z \in D^{\delta}\left(c_{j}\right)$ we have

$$
\left|f_{k}(z)\right| \leq C\left|\frac{g_{k}(z)}{z-c_{j}}\right| \rho\left(z_{k}\right)
$$

Estimating like in (iii) in the proof of Theorem 18 we get $\left|f_{k}(z)\right| \leq C M(\Lambda) e^{\phi(z)-\phi\left(z_{n}\right)}$, as desired.

In case $z_{k}=\sigma_{k} \in \Sigma$, use again that $\Lambda$ plus one point is $\mathcal{F}_{\phi}^{2}$-interpolating and start with $g_{k} \in \mathcal{F}_{\phi}^{2} \subset \mathcal{F}_{\phi}^{\infty}$ such that $g_{k}\left(\sigma_{k}\right)=1, g_{k}\left(\lambda_{j}\right)=0$ for all $j$. Then proceed as before.

Lemma 46. $Z \in \operatorname{Int} \mathcal{F}_{\phi}^{\infty}$. 
Proof. Given $v=\left\{v_{k}\right\}_{k} \in \ell_{\phi}^{\infty}(Z)$ consider the pseudo-extension

$$
E(v)(z)=\sum_{k=1}^{\infty} v_{k} f_{k}(z)
$$

Let us see first that $E(v) \in \mathcal{F}_{\phi}^{\infty}$. By (c) above and Lemma 6 we see that for any $z \in \mathbb{C}$

$$
e^{-\phi(z)}|E(v)(z)| \lesssim \sum_{k=1}^{\infty}\left|v_{k}\right| e^{-\phi\left(z_{k}\right)} \frac{1}{1+d_{\phi}^{M}\left(z, z_{k}\right)} \lesssim\|v\|_{\ell_{\phi}^{\infty}(Z)} .
$$

Let $R$ denote the restriction operator from $\mathcal{F}_{\phi}^{\infty}$ to $\ell_{\phi}^{\infty}(Z)$. In order to see that $Z$ is in Int $\mathcal{F}_{(1+\varepsilon) \phi}^{\infty}$ it will be enough to prove that $\|R E-I\|_{o p}<1$, since then $(R E)^{-1}=I+\sum_{k=1}^{\infty}(R E-I)^{k}$ converges and $E(R E)^{-1}$ defines an inverse to $R$.

By Lemma 45(b) and (c)

$$
\begin{aligned}
& \|R E(v)-v\|_{\ell_{\phi}^{\infty}(Z)}=\left\|\left\{\sum_{k: k \neq j} v_{k} f_{k}\left(z_{j}\right)\right\}_{j \in \mathbb{N}}\right\|_{\ell_{\phi}^{\infty}(Z)} \\
& \quad \leq \sup _{j \in \mathbb{N}} e^{-\phi\left(z_{j}\right)} \sum_{k: z_{j} \notin D^{1 / \varepsilon}\left(z_{k}\right)}\left|v_{k}\right|\left|f_{k}\left(z_{j}\right)\right| \leq C M(\Lambda)\|v\|_{\ell_{\phi}^{\infty}(Z)} \sum_{k: z_{j} \notin D^{1 / \varepsilon}\left(z_{k}\right)} \frac{1}{d_{\phi}^{m}\left(z_{j}, z_{k}\right)}
\end{aligned}
$$

By Lemma 4 and Corollary 7 , if $m$ is big and $\varepsilon$ is small enough we have

$$
\|R E(v)-v\|_{\ell_{\phi}^{\infty}(Z)} \leq 1 / 2\|v\|_{\ell_{\phi}^{\infty}(Z)},
$$

thus $\|R E-I\|_{o p}<1 / 2$, as desired.

By this Lemma and the results above we have $\mathcal{D}_{\Delta \phi}^{+}(Z) \leq 1 / 2 \pi$, i.e for all $\delta>0$ there exists $s_{0}$ such that for all $z \in \mathbb{C}$ and $s>s_{0}$

$$
n_{\Lambda}(z, s \rho(z))+n_{\Sigma}(z, s \rho(z)) \leq(1 / 2 \pi+\delta) \mu\left(D^{s}(z)\right) .
$$

By Lemma 37, $\mathcal{D}_{\Delta \phi}^{-}(\Sigma)=\varepsilon / \pi$, thus for all $\delta>0$ there exists $t_{0}$ such that for all $t>t_{0}$

$$
n_{\Sigma}(z, t \rho(z)) \geq(\varepsilon / \pi-\delta) \mu\left(D^{t}(z)\right) \quad z \in \mathbb{C} .
$$

This shows that for $\delta>0$ and $s$ big enough

$$
n_{\Lambda}(z, s \rho(z)) \leq\left(\frac{1-2 \varepsilon}{2 \pi}+2 \delta\right) \mu\left(D^{s}(z)\right) \quad z \in \mathbb{C},
$$

hence $\mathcal{D}_{\Delta \phi}^{+}(\Lambda)<1 / 2 \pi$.

\section{ApPendix. Alternative CONSTRUCtion of PEAK FUnCTIONS. (TheOREM 18)}

It will be enough to prove that for any $\phi$ there exist $C, \delta>0$ such that for all $\eta \in \mathbb{C}$ there is $P_{\eta}$ holomorphic with $P_{\eta}(\eta)=1$ and

$$
\left|P_{\eta}(z)\right| \leq C e^{\phi(z)-\phi(\eta)} \min \left\{1,\left(\frac{\rho(\eta)}{|z-\eta|}\right)^{\delta}\right\}
$$


since then we can apply this to $\varepsilon \delta / m \phi(z)$, take the $m$-th power and use Lemma 4 to conclude.

We claim that there exists $f_{\eta}$ holomorphic with $f_{\eta}(\eta)=0, f_{\eta}^{\prime}(\eta)=1$ and

$$
\left|f_{\eta}(z)\right| \lesssim e^{\phi(z)-\phi(\eta)} \rho^{2}(\eta) / \rho(z) .
$$

Once this is proved we take $P_{\eta}(z)=f_{\eta}(z) /(z-\eta)$ and use Lemma 2 to deduce that

$$
\left|P_{\eta}(z)\right| \lesssim e^{\phi(z)-\phi(\eta)} \frac{\rho(\eta)}{|z-\eta|}\left(\frac{|z-\eta|}{\rho(\eta)}\right)^{1-\delta}=e^{\phi(z)-\phi(\eta)}\left(\frac{\rho(\eta)}{|z-\eta|}\right)^{\delta} \quad z \notin D(\eta) .
$$

In order to construct the function $f_{\eta}$ define first

$$
F(z)=(z-\eta) \mathcal{X}\left(\frac{|z-\eta|^{2}}{\rho^{2}(\eta)}\right) e^{H_{\eta}(z)}
$$

where $H_{\eta}$ is a holomorphic function such that $R e H_{\eta}=h_{\eta}$ (see Lemma 13) and $\mathcal{X}$ is a smooth cut-off function with $\mathcal{X} \equiv 1$ for $|\zeta|<1, \mathcal{X} \equiv 0$ for $|\zeta| \geq 2$ and $\left|\mathcal{X}^{\prime}\right|$ bounded.

Notice that by construction and by Lemma 13 , we have

$$
\rho(z)|F(z)| e^{-\phi(z)} \lesssim \rho^{2}(\eta) e^{-\phi(\eta)} .
$$

Lemma 47. There exists $u$ solution to $\bar{\partial} u=\bar{\partial} F$ such that $u(\eta)=\partial u(\eta)=0$ and

$$
\left\|u e^{-\phi} \rho\right\|_{L^{\infty}(\mathbb{C})} \leq C \rho^{2}(\eta) e^{-\phi(\eta)}
$$

Once this is proved we take $f_{\eta}=F-u$ and we are done.

Proof. First we show that there exists a solution $u$ as in the statement but satisfying an analogous $L^{2}$ estimate instead of the $L^{\infty}$ one. We use Hörmander's theorem [Hör94]: for every $\psi$ subharmonic in $\mathbb{C}$ there exists a solution $u$ to $\bar{\partial} u=\bar{\partial} F$ such that

$$
\int_{\mathbb{C}}|u|^{2} e^{-2 \psi} \leq C \int_{\mathbb{C}}|\bar{\partial} F|^{2} \frac{e^{-2 \psi}}{\Delta \psi}
$$

Define $\psi=\phi+2 v$, where

$$
v(z)=\log |z-\eta|-\frac{1}{\mu\left(D^{s}(\eta)\right)} \int_{D^{s}(\eta)} \log |z-\zeta| \Delta \phi(\zeta) d m(\zeta) .
$$

Take $s$ so that $\mu\left(D^{s}(\eta)\right)=8 \pi$. By the doubling condition there exists $c$ depending only on the doubling constant $C_{\Delta \phi}$ such that $s \leq c$. Then

$$
\Delta \psi \geq \Delta \phi-\frac{4 \pi}{\mu\left(D^{s}(\eta)\right)} \Delta \phi=\frac{1}{2} \Delta \phi \simeq \rho^{-2} .
$$

By construction $v$ is bounded above. Notice also that there exists $C>0$ (independent of $\eta$ ) such that $-v(z) \leq C$ for all $z \in \operatorname{supp}(\bar{\partial} F)$. Since $|\bar{\partial} F| \lesssim e^{h_{\eta}}$, we deduce from Hörmander's estimate and Lemma 13 that

$$
\int_{\mathbb{C}}|u|^{2} e^{-2 \phi} \leq \int_{\mathbb{C}}|u|^{2} e^{-2 \psi} \leq C \int_{D^{2}(\eta) \backslash D(\eta)} e^{2 h_{\eta}} e^{-2 \psi} \rho^{2} \lesssim \rho^{4}(\eta) e^{-2 \phi(\eta)} .
$$


On the other hand

$$
e^{-2 \psi(z)} \simeq|z-\eta|^{-4} \quad \text { for } \quad|z-\eta| \leq \epsilon \rho(\eta),
$$

thus necessarily $u(\eta)=\partial u(\eta)=0$.

Let us see now that $u$ satisfies also the $L^{\infty}$ estimate. For any $z \in \operatorname{supp}(\bar{\partial} F)$ define

$$
U(\zeta)=\frac{K \rho(z)}{\rho^{2}(\eta) e^{-\phi(\eta)}} u(\zeta)
$$

where $K>0$ will be chosen later on. Then

$$
\int_{D(z)}|U(\zeta)|^{2} e^{-2 \phi(\zeta)} \leq \frac{\rho^{2}(z)}{\rho^{4}(\eta) e^{-2 \phi(\eta)}}\|u\|_{L^{2}\left(e^{-\phi}\right)}^{2} \lesssim \rho^{2}(z) .
$$

Also, since $\rho(\zeta) \simeq \rho(\eta)$ on $\operatorname{supp}(\bar{\partial} F)$, we have

$$
\rho(z) \sup _{\zeta \in D(z)}|\bar{\partial} U(\zeta)| e^{-\phi(\zeta)}=\sup _{\zeta \in D(z)} \frac{K \rho^{2}(z)}{\rho^{2}(\eta) e^{-\phi(\eta)}}|\bar{\partial} F(\zeta)| e^{-\phi(\zeta)} \lesssim 1
$$

We choose $K$ (independent of $z$ ) so that

(a) $\frac{1}{\rho^{2}(z)} \int_{D(z)}|U(\zeta)|^{2} e^{-2 \phi(\zeta)} \leq 1$,

(b) $\rho(z) \sup _{\zeta \in D(z)}|\bar{\partial} U(\zeta)| e^{-\phi(\zeta)} \leq 1$

We will be done as soon as we prove that

$$
|U(z)| e^{-\phi(z)} \leq C
$$

This is consequence of [Ber97, Lemma 3.1] applied to the function $V(\zeta)=u(\rho(z) \zeta+z)$. Defining $\phi_{z}(\zeta)=\phi(\rho(z) \zeta+z)$ and changing to the variable $w=\rho(z) \zeta+z$ we see that

$$
\int_{\mathbb{D}}|V(\zeta)|^{2} e^{-2 \phi_{z}(\zeta)} d m(\zeta)=\int_{D(z)}|U(w)|^{2} e^{-2 \phi(w)} \frac{d m(w)}{\rho^{2}(z)} \leq 1
$$

and

$$
\sup _{\zeta \in \mathbb{D}}|\bar{\partial} V(\zeta)|^{2} e^{-2 \phi_{z}(\zeta)}=\sup _{w \in D(\eta)}|\bar{\partial} U(w)|^{2} e^{-2 \phi(w)} \rho(z) \leq 1 .
$$

Thus, by [Ber97, Lemma 3.1] $|V(0)|^{2} e^{-\phi_{z}(0)} \leq C e^{-a_{\phi_{z}}}$, where

$$
a_{\phi_{z}}=\sup \left\{\psi(0): \psi \leq 0, \Delta \psi=\Delta \phi_{\eta}\right\} \text {. }
$$

Defining $v$ so that $\psi(z)=v(\rho(z) \zeta+z)$ we see that

$$
a_{\phi_{z}}=\sup \{v(z): v \leq 0: \Delta v=\Delta \phi\} .
$$

The function $v(w)=\phi(w)-h_{z}(w)-\phi(z)-A$ is negative in $D(z)$ if $A$ is big enough (Lemma 13) and $v(z)=-A$. Hence $a_{\phi_{z}} \geq-A$ and $|U(z)|^{2} e^{-2 \phi(z)}=|V(0)|^{2} e^{-2 \phi_{z}(0)} \leq C e^{A}$, as desired. 


\section{REFERENCES}

[Ber97] Bo Berndtsson, Uniform estimates with weights for the $\bar{\partial}$-equation, J. Geom. Anal. 7 (1997), no. 2, 195215. MR 99h:32018

[Beu89] Arne Beurling, The collected works of Arne Beurling. Vol. 2, Birkhäuser Boston Inc., Boston, MA, 1989, Harmonic analysis, Edited by L. Carleson, P. Malliavin, J. Neuberger and J. Wermer. MR 92k:01046b

[BL95] Carlos A. Berenstein and Bao Qin Li, Interpolating varieties for spaces of meromorphic functions, J. Geom. Anal. 5 (1995), no. 1, 1-48. MR 96b:30064

[BOC95] Bo Berndtsson and Joaquim Ortega-Cerdà, On interpolation and sampling in Hilbert spaces of analytic functions, J. Reine Angew. Math. 464 (1995), 109-128. MR 96g:30070

[Chr91] Michael Christ, On the $\bar{\partial}$ equation in weighted $L^{2}$ norms in $\mathbb{C}^{1}$, J. Geom. Anal. 1 (1991), no. 3, 193-230. MR 92j:32066

[Dra01] David Drasin, Approximation of subharmonic functions with applications, Approximation, complex analysis, and potential theory (Montreal, QC, 2000), Kluwer Acad. Publ., Dordrecht, 2001, pp. 163-189. MR 1873588

[FS89] John Erik Fornæss and Nessim Sibony, Construction of P.S.H. functions on weakly pseudoconvex domains, Duke Math. J. 58 (1989), no. 3, 633-655. MR 90m:32034

[Hör94] Lars Hörmander, Notions of convexity, Birkhäuser Boston Inc., Boston, MA, 1994. MR 95k:00002

[Lan67] H. J. Landau, Necessary density conditions for sampling and interpolation of certain entire functions, Acta Math. 117 (1967), 37-52. MR 36 \#5604

[Lin01] Niklas Lindholm, Sampling in weighted $L^{p}$ spaces of entire functions in $\mathbb{C}^{n}$ and estimates of the Bergman kernel, J. Funct. Anal. 182 (2001), no. 2, 390-426. MR 1828799

[LM01] Yuriĭ Lyubarskiĭ and Eugenia Malinnikova, On approximation of subharmonic functions, J. Anal. Math. 83 (2001), 121-149. MR 2002b:30043

[LS94] Yuriǐ I. Lyubarskiı̌ and Kristian Seip, Sampling and interpolation of entire functions and exponential systems in convex domains, Ark. Mat. 32 (1994), no. 1, 157-193. MR 95j:30025

[LY91] V. I. Lutsenko and R. S. Yulmukhametov, Generalizations of the Wiener-Paley theorem to functionals in Smirnov spaces, Trudy Mat. Inst. Steklov. 200 (1991), 245-254. MR 92j:46048

[Lyu88] Yu. I. Lyubarskiı̆, The Paley-Wiener theorem for convex sets, Izv. Akad. Nauk Armyan. SSR Ser. Mat. 23 (1988), no. 2, 163-172, 194. MR 90b:30029

[Mat95] Pertti Mattila, Geometry of sets and measures in Euclidean spaces, Cambridge University Press, Cambridge, 1995, Fractals and rectifiability. MR 96h:28006

[MT00] Xavier Massaneda and Pascal J. Thomas, Interpolating sequences for Bargmann-Fock spaces in $\mathbb{C}^{n}$, Indag. Math. (N.S.) 11 (2000), no. 1, 115-127. MR 2001i:32018

[OC99] Joaquim Ortega-Cerdà, Multipliers and $\bar{\partial}$ estimates, Rev. Mat. Iberoamericana, 18 (2002), no. 2, 355377.

[OCS98] Joaquim Ortega-Cerdà and Kristian Seip, Beurling-type density theorems for weighted $L^{p}$ spaces of entire functions, J. Anal. Math. 75 (1998), 247-266. MR 2000k:46030

[RS95] Jayakumar Ramanathan and Tim Steger, Incompleteness of sparse coherent states, Appl. Comput. Harmon. Anal. 2 (1995), no. 2, 148-153. MR 96b:81049

[Sei92] Kristian Seip, Density theorems for sampling and interpolation in the Bargmann-Fock space. I, J. Reine Angew. Math. 429 (1992), 91-106. MR 93g:46026a

[Sei93] _ Beurling type density theorems in the unit disk, Invent. Math. 113 (1993), no. 1, 21-39. MR 94g:30033

[Squ83] William A. Squires, Geometric condition for universal interpolation in $\hat{\mathcal{E}}^{\prime}$, Trans. Amer. Math. Soc. 280 (1983), no. 1, 401-413. MR 84h:30058

[SS61] H. S. Shapiro and A. L. Shields, On some interpolation problems for analytic functions, Amer. J. Math. 83 (1961), 513-532. MR 24 \#A3280

[SW92] Kristian Seip and Robert Wallstén, Density theorems for sampling and interpolation in the BargmannFock space. II, J. Reine Angew. Math. 429 (1992), 107-113. MR 93g:46026b 
[Yul85] R. S. Yulmukhametov, Approximation of subharmonic functions, Anal. Math. 11 (1985), no. 3, $257-282$. MR 88a:31002

Dept. Matemàtiques, Universitat Autonoma de Barcelona, 08193 Bellaterra (Barcelona), SPAIN

Email address: nmarco@mat.uab.es

Dept. Matemàtica Aplicada i AnÀlisi, Universitat de BARCElona, Gran Via 585, 08071 BarCELONA, SPAIN

Email address: xavieremat.ub.es

Dept. MatemÀtica Aplicada i AnÀlisi, Universitat de Barcelona, Gran Via 585, 08071 BarCELONA, SPAIN

Email address: quim@mat . ub.es 